\title{
Quantum transfer-matrices for the sausage model
}

\author{
Vladimir V. Bazhanov, ${ }^{a}$ Gleb A. Kotousov ${ }^{a, b}$ and Sergei L. Lukyanov ${ }^{b, c}$ \\ ${ }^{a}$ Department of Theoretical Physics, Research School of Physics and Engineering, \\ Australian National University, \\ Canberra, ACT 2601, Australia \\ ${ }^{b}$ NHETC, Department of Physics and Astronomy, Rutgers University, \\ Piscataway, NJ 08855-0849, U.S.A. \\ ${ }^{c}$ L.D. Landau Institute for Theoretical Physics, \\ Chernogolovka 142432, Russia \\ E-mail: vladimir.bazhanov@anu.edu.au, kotoousov@physics.rutgers.edu, \\ sergei@physics.rutgers.edu
}

ABSTRACT: In this work we revisit the problem of the quantization of the two-dimensional $O(3)$ non-linear sigma model and its one-parameter integrable deformation - the sausage model. Our consideration is based on the so-called ODE/IQFT correspondence, a variant of the Quantum Inverse Scattering Method. The approach allowed us to explore the integrable structures underlying the quantum $O(3)$ /sausage model. Among the obtained results is a system of non-linear integral equations for the computation of the vacuum eigenvalues of the quantum transfer-matrices.

KEywords: Field Theories in Lower Dimensions, Integrable Field Theories, Lattice Integrable Models, Sigma Models

ARXiv EPrint: 1706.09941

To the memory of Ludwig Dmitrievich Faddeev. 


\section{Contents}

1 Introduction $\quad 2$

2 Chiral transfer-matrices for the cigar $\quad 7$

2.1 Wilson loops for the long sausage 8

2.2 Definition and basic properties of the chiral transfer-matrices 10

$\begin{array}{lll}2.3 & \text { Basic facts about the quantum cigar } & 15\end{array}$

3 Chiral transfer-matrix for $\mathbb{Z}_{n}$ parafermions 19

$\begin{array}{lll}3.1 & \text { Bosonization of } \mathbb{Z}_{n} \text { parafermions } & 20\end{array}$

3.2 Discretization of the chiral transfer-matrix 22

4 Spectrum of the chiral transfer-matrix $\quad 27$

$\begin{array}{lll}4.1 & \text { Operators } \zeta_{ \pm}(\theta) & 28\end{array}$

4.2 ODE/IQFT correspondence for the vacuum eigenvalues 31

4.3 ODE/IQFT correspondence for the full spectrum 36

4.4 Operators $\beta_{ \pm}(\theta)$ and $\alpha_{ \pm}(\theta) \quad 37$

4.5 NLIE for the vacuum eigenvalues 41

5 Integrable structures in the sausage $\quad 45$

$\begin{array}{ll}5.1 \text { Basic facts about the quantum sausage } & 45\end{array}$

5.2 NLIE for the $k$-vacuum eigenvalues in the sausage model 50

$\begin{array}{lll}5.3 & \mathbb{A}, \mathbb{B} \text { and } \mathbb{T} & 56\end{array}$

5.4 ODE/IQFT for the sausage model 63

$\begin{array}{lll}6 & \text { Discussion } & 66\end{array}$

A Scaling behaviour of discretized chiral transfer-matrices $\quad 68$

$\begin{array}{ll}\text { B Scaling behaviour of Bethe roots for the vacuum state } & 71\end{array}$

$\begin{array}{ll}\text { C Derivation of NLIE } & 76\end{array}$

$\begin{array}{lr}\text { D Modified NLIE for oscillating asymptotics } & 80\end{array}$

$\begin{array}{lll}\text { E Two-particle } S \text {-matrix for the sausage model } & 81\end{array}$ 


\section{Introduction}

Integrability is a traditional area of mathematical physics having a long history. The notion is relatively well understood in finite dimensional systems: it requires the system to have exactly $n$ isolating and commuting integrals of motion where $n$ is the number of degrees of freedom. In the context of $1+1$ dimensional field theory, where the continuous number of degrees of freedom makes the traditional definition insufficient, a suitable paradigm of integrability was also discovered. The key ingredient in this case is a Lie algebra-valued world sheet connection involving an analytic spectral parameter, whose flatness condition is equivalent to the classical equations of motion. Since the Wilson loops

$$
T=\operatorname{Tr} \overleftarrow{\mathcal{P}} \exp \int_{C} \boldsymbol{A}
$$

remain unchanged under continuous deformations of the integration contour (see figure 1), they generate an infinite family of conserved quantities which can be used to solve the field theory within the framework of the inverse scattering method. At the end of the seventies the Quantum Inverse Scattering Method (QISM) was proposed [1]. The approach was inspired by the pioneering works of Baxter on lattice statistical systems [2,3] and based on the study of the common spectrum of the transfer-matrices ( $T$-operators) - the quantum counterpart of the classical Wilson loops. The original formulation of the QISM was restricted to the so-called "ultralocal" models where the elementary transport matrices $\boldsymbol{M}_{n}=$ $\overleftarrow{\mathcal{P}} \exp \int_{x_{n}}^{x_{n+1}} \boldsymbol{A}$ commute for different segments of the discretized path. The study of the algebra of the ultralocal operators $\boldsymbol{M}_{n}$ led to the discovery of new remarkable mathematical structures collectively known today as the Yang-Baxter algebras. The most studied class of integrable models is the one where the Yang-Baxter algebra of the ultralocal operators $\boldsymbol{M}_{n}$ admits a finite-dimensional representation. In this case the discretized quantum system can be interpreted as an exactly soluble statistical model whose solution can be obtained by means of the Bethe ansatz method. The solution of the continuous QFT is achieved by taking a proper scaling limit. An archetype of this scenario is the sine-Gordon model, while the corresponding statistical system is known as the inhomogeneous 6-vertex model [4].

However the QISM fails when it is applied to many interesting systems including classically integrable Nonlinear Sigma Models (NLSM). The origin of the problem can be illustrated by the $O(3)$ NLSM governed by the Lagrangian

$$
\mathcal{L}=\frac{1}{2}\left(\left(\partial_{t} \boldsymbol{n}\right)^{2}-\left(\partial_{x} \boldsymbol{n}\right)^{2}\right),
$$

where the 3 -dimensional vector $\boldsymbol{n}$ is subject to the constraint $\boldsymbol{n} \cdot \boldsymbol{n}=1$. In this case the light-cone components of the flat connection, $\boldsymbol{A}_{ \pm} \equiv \frac{1}{2}\left(\boldsymbol{A}_{t} \pm \boldsymbol{A}_{x}\right)$, are given by the relations $[5,6]$

$$
\boldsymbol{A}_{ \pm}=\frac{2}{1 \pm \zeta}\left[\partial_{ \pm} \check{\boldsymbol{n}}, \check{\boldsymbol{n}}\right], \quad \check{\boldsymbol{n}}=n_{a} \mathrm{t}_{a} \in \mathfrak{s u}(2)
$$

where $\partial_{ \pm}=\frac{1}{2}\left(\partial_{t} \pm \partial_{x}\right)$ while $\check{\boldsymbol{n}}$ is built from the components of the unit vector $\boldsymbol{n}$ and $\boldsymbol{t}_{a}$ :

$$
\left[\mathrm{t}_{a}, \mathrm{t}_{b}\right]=\mathrm{i} \varepsilon_{a b c} \mathrm{t}_{c} .
$$




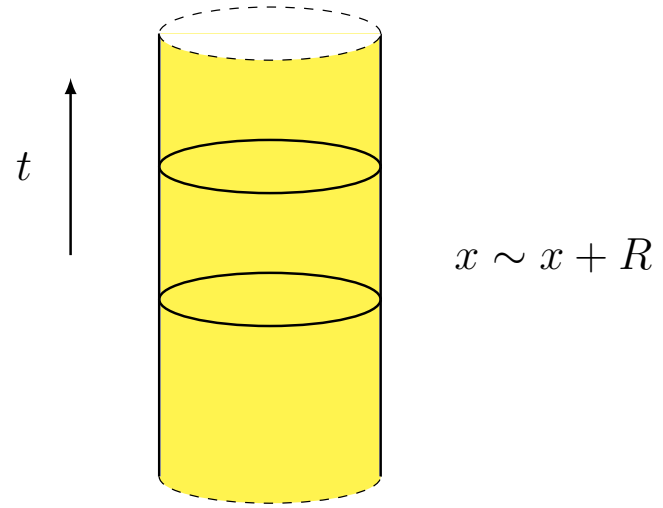

Figure 1. The integration contour for the Wilson loop can be moved freely along the space-time cylinder.

With periodic boundary conditions, $\boldsymbol{n}(t, x+R)=\boldsymbol{n}(t, x)$, the flat connection turns out to be a single-valued 1-form on the space-time cylinder and the Wilson loops (1.1) are conserved charges for any value of the spectral parameter $\zeta$. The equal time Poisson brackets of (1.3) respect the general structure

$$
\left\{\boldsymbol{A}_{\mu}(x) \otimes \boldsymbol{A}_{\nu}(y)\right\}=\boldsymbol{C}_{\mu \nu}^{(0)}(x) \delta(x-y)+\boldsymbol{C}_{\mu \nu}^{(1)}(x) \delta^{\prime}(x-y),
$$

where $\boldsymbol{C}_{\mu \nu}^{(i)}(x)$ are local fields built from $n_{a}$ and $\partial_{t} n_{a}$ and take values in the tensor product $\mathfrak{s u}(2) \otimes \mathfrak{s u}(2)$. Because of the derivative of the $\delta$-function, relations of this type are usually referred to as non-ultralocal. The presence of such terms creates serious problems with the rigorous proof of Poisson commutativity of the conserved charges (1.1) for different values of the spectral parameter. This, in turn, hampers the first-principles quantization of the model.

The problem with non-ultralocality is a general issue for integrable NLSM and is a major obstacle which prevents the incorporation of these models into the framework of the QISM. This was first observed in attempts to quantize the principal chiral field and $O(N)$ models. In the case of the $O(4)$-model (i.e., the model (1.2) with $\boldsymbol{n} \in \mathbb{S}^{3}$ ), Polyakov and Wiegmann [7] and later Faddeev and Reshetikhin [8] managed to bypass the problem with non-ultralocality. In both works, the NLSM was replaced by a different model satisfying the ultralocality condition; Polyakov and Wiegmann considered a model with $N_{f}$ fermion flavors, whereas Faddeev and Reshetikhin focused on a certain spin- $S$ chain. They studied the thermodynamics using the Bethe ansatz technique and gained valuable results for the $O(4)$ sigma model through the large $N_{f}$ and $S \rightarrow \infty$ limit, respectively. Both limiting procedures yielded the same system of Thermodynamic Bethe Ansatz Equations (TBA), which was then justified by a comparison with perturbative calculations and the exact results from the $S$-matrix bootstrap [9]. In the next 30 years an enormous amount of TBA systems were discovered. However, in spite of significant achievements in their study, the original problem of the construction of quantum transfer-matrices (including the calculation of their spectrum) has remained unsolved even for the $O(3)$ and $O(4)$ NLSM. 


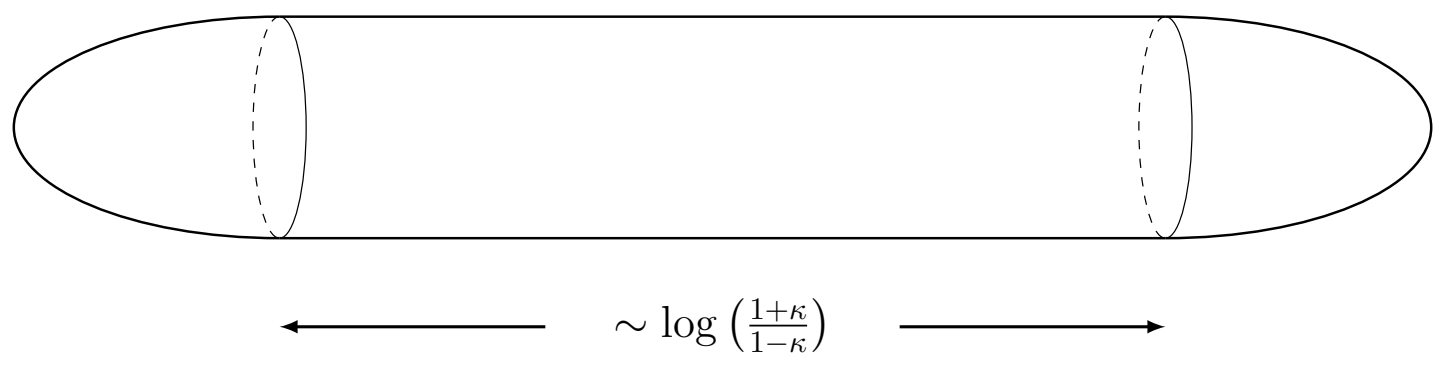

Figure 2. A depiction of the target manifold of the NLSM (1.6) for $1-\kappa \ll 1$.

Is the non-ultralocal structure (1.5) an immanent property of an integrable NLSM, or is it just the result of an awkward choice of gauge for the flat connection? Unfortunately, we do not know the answer for the most interesting models, however in the case of the $O(3)$ sigma model, it turns out that the "ultralocal" gauge does exist [10]. ${ }^{1}$ Further, we found that such a gauge also exists for the theory described by the Lagrangian

$$
\mathcal{L}=\frac{1}{2} \frac{\partial_{\mu} \boldsymbol{n} \partial^{\mu} \boldsymbol{n}}{\kappa^{-1}-\kappa n_{3}^{2}}
$$

This theory also belongs to the class of NLSM, and, assuming $0<\kappa<1$, the corresponding target space is topologically the two-sphere. As $\kappa \rightarrow 0$ one can neglect the second term in the denominator which results in the $O(3)$ Lagrangian multiplied by $\kappa$. Hence, the model (1.6) is the one parameter deformation of the $O(3)$ sigma model. It is colloquially known as the "sausage model" since for $\kappa \rightarrow 1^{-}$the target manifold can be pictured as a long sausage with length $\propto \log \left(\frac{1+\kappa}{1-\kappa}\right)$ (see figure 2). The sausage model was introduced in the work [14] where strong evidence for its quantum integrability was presented. Its zero curvature representation was found in ref. [15]. Remarkably, with the proper gauge transformation, the flat connection can be chosen to satisfy the ultralocal Poisson bracket relations

$$
\begin{aligned}
& \left\{\boldsymbol{A}_{ \pm}(x \mid \mu) \otimes \boldsymbol{A}_{ \pm}\left(x^{\prime} \mid \mu^{\prime}\right)\right\}= \pm\left[\boldsymbol{A}_{ \pm}(x \mid \mu) \otimes \mathbf{1}+\mathbf{1} \otimes \boldsymbol{A}_{ \pm}\left(x^{\prime} \mid \mu^{\prime}\right), \boldsymbol{r}\left(\mu / \mu^{\prime}\right)\right] \delta\left(x-x^{\prime}\right) \\
& \left\{\boldsymbol{A}_{ \pm}(x \mid \mu) \otimes, \boldsymbol{A}_{\mp}\left(x^{\prime} \mid \mu^{\prime}\right)\right\}=0 .
\end{aligned}
$$

Here

$$
\boldsymbol{r}(\mu)=\frac{1}{\mu-\mu^{-1}}\left(2 \mathrm{t}_{1} \otimes \mathrm{t}_{1}+2 \mathrm{t}_{2} \otimes \mathrm{t}_{2}+\left(\mu+\mu^{-1}\right) \mathrm{t}_{3} \otimes \mathrm{t}_{3}\right),
$$

and the dependence on the multiplicative spectral parameter $\mu$ is made explicit ( $\mu$ substitutes the auxiliary parameter $\zeta$ in eq. (1.3)).

To present explicit formulae for the ultralocal flat connection, we first resolve the constraint $\boldsymbol{n}^{2}=1$ by means of the stereographic projection. It allows the triple $\boldsymbol{n}=$ $\left(n_{1}, n_{2}, n_{3}\right)$ to be substituted by the complex unconstrained field $Q$,

$$
\mathrm{e}^{Q}=\sqrt{\frac{1-n_{3}}{1+n_{3}} \frac{n_{1}+\mathrm{i} n_{2}}{n_{1}-\mathrm{i} n_{2}}}, \quad \mathrm{e}^{Q^{*}}=\sqrt{\frac{1-n_{3}}{1+n_{3}} \frac{n_{1}-\mathrm{i} n_{2}}{n_{1}+\mathrm{i} n_{2}}} .
$$

\footnotetext{
${ }^{1}$ It is worth noting that a transition to an ultralocal gauge has previously been utilised for some other important but apparently non-ultralocal systems, such as the (quantum) KdV theory [11] and its discretized analogs $[12,13]$.
} 
The pair of momenta $\left(\Pi, \Pi^{*}\right)$ canonically conjugate to $\left(Q, Q^{*}\right)$ are given by the relations

$$
\Pi=\frac{1-n_{3}^{2}}{2\left(\kappa^{-1}-\kappa n_{3}^{2}\right)} \partial_{t} Q^{*}, \quad \Pi^{*}=\frac{1-n_{3}^{2}}{2\left(\kappa^{-1}-\kappa n_{3}^{2}\right)} \partial_{t} Q,
$$

where $n_{3}=-\tanh \left(\frac{1}{2}\left(Q+Q^{*}\right)\right)$. Let us also introduce the shortcut notations

$$
\Pi_{+}=\Pi+\frac{1-n_{3}^{2}}{2\left(\kappa^{-1}-\kappa n_{3}^{2}\right)} \partial_{x} Q^{*}, \quad \Pi_{-}=\Pi^{*}-\frac{1-n_{3}^{2}}{2\left(\kappa^{-1}-\kappa n_{3}^{2}\right)} \partial_{x} Q
$$

and the field independent element of $\mathfrak{s l}(2, \mathbb{C})$

$$
\boldsymbol{a}(\mu)=f(\mu) \mathrm{t}_{3}+\mathrm{i} g(\mu) \mathrm{t}_{1},
$$

where

$$
\begin{aligned}
& f(\mu)=\frac{(1-\kappa) \mu+(1+\kappa) \mu^{-1}}{(1-\kappa) \mu-(1+\kappa) \mu^{-1}} \\
& g(\mu)=\frac{2 \sqrt{1-\kappa^{2}}}{(1-\kappa) \mu-(1+\kappa) \mu^{-1}} .
\end{aligned}
$$

It is straightforward to check now that the formulae ${ }^{2}$

$$
\begin{aligned}
& \boldsymbol{A}_{+}=+\Pi_{+} \mathrm{e}^{Q \mathrm{t}_{3}} \boldsymbol{a}(\mu) \mathrm{e}^{-Q \mathrm{t}_{3}} \\
& \boldsymbol{A}_{-}=-\Pi_{-} \mathrm{e}^{\mathrm{i} \pi \mathrm{t}_{3}} \mathrm{e}^{-Q^{*} \mathrm{t}_{3}} \boldsymbol{a}\left(\mu^{-1}\right) \mathrm{e}^{Q^{*} \mathrm{t}_{3}} \mathrm{e}^{-\mathrm{i} \pi \mathrm{t}_{3}}
\end{aligned}
$$

define the flat connection, i.e.,

$$
\left[\partial_{+}-\boldsymbol{A}_{+}, \partial_{-}-\boldsymbol{A}_{-}\right]=0
$$

satisfying the ultralocality conditions (1.7).

Having an explicit formula for the ultralocal flat connection we may turn to the construction of the time-independent Wilson loops. In fact their construction requires some assumptions to be made. First of all in writing eq. (1.1) it is assumed that the connection is single valued on the space-time cylinder. In the case under consideration this can be achieved by imposing periodic boundary conditions on the unit vector $\boldsymbol{n}$. Such boundary conditions look natural for the $O(3)$ NLSM, since they preserve the global invariance of the Lagrangian. In the case of the sausage model, the $O(3)$ symmetry is broken down to $\mathrm{U}(1)$ and we will consider more general boundary conditions

$$
n_{3}(t, x+R)=n_{3}(t, x), \quad n_{ \pm}(t, x+R)=\mathrm{e}^{ \pm 2 \pi \mathrm{i} k} n_{ \pm}(t, x) \quad\left(n_{ \pm} \equiv n_{1} \pm \mathrm{i} n_{2}\right)
$$

\footnotetext{
${ }^{2}$ Notice that the connection in the ultralocal gauge takes values in the complexification of the Lie algebra $\mathfrak{s u}(2)$. This should not worry us because only reality conditions imposed on gauge invariant quantities are a subject of interest and importance. In what follows we'll prefer to use the Cartan-Weyl generators $\mathrm{h}=2 \mathrm{t}_{3}, \mathrm{e}_{ \pm}=\mathrm{t}_{1} \pm \mathrm{it} \mathrm{t}_{2}$ :

instead of $t_{a}$ (1.4).

$$
\left[\mathrm{h}, \mathrm{e}_{ \pm}\right]= \pm 2 \mathrm{e}_{ \pm}, \quad\left[\mathrm{e}_{+}, \mathrm{e}_{-}\right]=\mathrm{h}
$$
}


which depend on the twist parameter $k \sim k+1$. In terms of the real and imaginary part of the complex field $Q$,

$$
Q=\phi+\mathrm{i} \alpha
$$

the twisted boundary conditions are equivalently given by

$$
\phi(t, x+R)=\phi(t, x), \quad \alpha(t, x+R)=\alpha(t, x)+2 \pi k .
$$

As it follows from eq. (1.14) the connection is not single valued on the space-time cylinder now,

$$
\boldsymbol{A}_{\mu}(t, x+R)=\mathrm{e}^{\mathrm{i} \pi k \mathrm{~h}} \boldsymbol{A}_{\mu}(t, x) \mathrm{e}^{-\mathrm{i} \pi k \mathrm{~h}},
$$

however with a little modification one can still introduce the time independent conserved charges

$$
T_{j}(\mu)=\operatorname{Tr}\left[\mathrm{e}^{-\mathrm{i} \pi k \mathrm{~h}} \boldsymbol{M}_{j}(\mu)\right], \quad \boldsymbol{M}_{j}=\pi_{j}\left[\overleftarrow{\mathcal{P}} \exp \int_{C} \mathrm{~d} x \boldsymbol{A}_{x}\right]
$$

Notice that the definition of the Wilson loop requires a choice of the representation of the Lie algebra and the subscript $j$ in (1.20) labels the representations $\pi_{j}$ of $\mathfrak{s l}(2)$. In our case we will focus on the finite dimensional irreps, so that in the standard convention $j=\frac{1}{2}, 1, \ldots$.

The ultralocal Poisson structure (1.7) implies that the monodromy matrix generates the classical Yang-Baxter Poisson algebra:

$$
\left\{\boldsymbol{M}_{j}(\mu) \otimes \boldsymbol{M}_{j^{\prime}}\left(\mu^{\prime}\right)\right\}=\left[\boldsymbol{M}_{j}(\mu) \otimes \boldsymbol{M}_{j^{\prime}}\left(\mu^{\prime}\right), \boldsymbol{r}_{j j^{\prime}}\left(\mu / \mu^{\prime}\right)\right]
$$

where $\boldsymbol{r}_{j j^{\prime}}=\pi_{j} \otimes \pi_{j^{\prime}}[\boldsymbol{r}]$. This, supplemented by the easily established property

$$
\left[\mathrm{e}^{-\mathrm{i} \pi k \mathrm{~h}} \otimes \mathrm{e}^{-\mathrm{i} \pi k \mathrm{~h}}, \boldsymbol{r}\left(\mu / \mu^{\prime}\right)\right]=0,
$$

implies the infinite set of relations

$$
\left\{T_{j}(\mu), T_{j^{\prime}}\left(\mu^{\prime}\right)\right\}=0 .
$$

Thus, we have arrived at formulae (1.21) and (1.23) which are key ingredients in the Hamiltonian approach to the inverse scattering method [16, 17].

When faced with the problem of quantizing the sausage NLSM, a simple idea that may come to mind is to discretize the path-ordered integral onto $N$ small segments. Due to ultralocality, the $N$ elementary transport matrices $\pi_{j}\left[\stackrel{\overleftarrow{\mathcal{P}}}{\exp } \int_{x_{n}}^{x_{n+1}} \mathrm{~d} x \boldsymbol{A}_{x}\right]$ satisfy the same type of Poisson bracket relation as (1.21) and Poisson commute for different segments. These relations can be formally quantized leading to a certain quantum Yang-Baxter algebra. The major problem now is to construct a suitable representation of this abstract algebraic structure. In the case under consideration, the representation is, in all likelihood, infinite dimensional even for finite $N$. At this moment, it is not clear for us how to construct and handle such representations, let alone take the scaling limit with $N \rightarrow \infty$.

In this work, we will try to avoid discretization as much as we can and mostly follow the so-called BLZ approach - the variant of the QISM developed in the series of works $[11,18,19]$. For integrable Conformal Field Theories (CFT), it was demonstrated 
that the $T$-operators can be constructed without any discretization procedure. Later it was observed that many deep properties of representations of Yang-Baxter algebras in integrable CFT can be encoded in the monodromies of certain linear Ordinary Differential Equations (ODE) [20-26]. These results were extended to massive Integrable Quantum Field Theories (IQFT) [27] (for recent developments, see also refs. [28-35]). The general relation of this type will be referred to in the paper as the ODE/IQFT correspondence.

Broadly speaking, the ODE/IQFT correspondence means that for a given IQFT the eigenvalues of the quantum $T$-operators are identified with certain connection coefficients for the system of equations,

$$
\boldsymbol{D}(\theta) \boldsymbol{\Psi}=0, \quad \overline{\boldsymbol{D}}(\theta) \boldsymbol{\Psi}=0,
$$

where $\boldsymbol{D}(\theta)$ and $\overline{\boldsymbol{D}}(\theta)$ stand for (singular) differential operators depending on the auxiliary parameter $\theta$ which is found to be a function of the original spectral parameter from the quantum theory. The system of ODE can be then interpreted as an auxiliary linear problem, whose compatibility condition, $[\boldsymbol{D}(\theta), \overline{\boldsymbol{D}}(\theta)]=0$, coincides with the zero-curvature representation for some classically integrable field theory. Thus the ODE/IQFT correspondence reduces the calculation of the spectrum of quantum transfer-matrices to a certain problem in the theory of classical integrable equations. The latter can be effectively treated by the inverse scattering transform method. This makes the ODE/IQFT correspondence a very powerful tool. In particular, it gives a practical way to make progress in the conceptual long standing problem of the quantization of integrable NLSM. The ultimate goal of this work is to demonstrate this for the case of the quantum sausage model.

\section{Chiral transfer-matrices for the cigar}

The BLZ approach $[11,18,19]$ begins with an analysis of the RG fixed point which controls the ultraviolet behaviour of the integrable QFT. With this in mind, let's take a quick look at the sausage NLSM (1.6). In the traditional path-integral quantization, the model should be equipped with a UV cutoff $\Lambda$. A consistent removal of the UV divergences requires that the "bare" coupling in the Lagrangian (1.6) be given a certain dependence on the cutoff momentum, i.e., $\kappa=\kappa(\Lambda)$. To the first perturbative order the RG flow equation is given by [14]

$$
\Lambda \frac{\partial \kappa}{\partial \Lambda}=\frac{\hbar}{2 \pi}\left(1-\kappa^{2}\right)+O\left(\hbar^{2}\right)
$$

where $\hbar$ stands for the (dimensionless) Planck constant. Integrating this equation leads to

$$
\frac{1-\kappa}{1+\kappa}=\left(E_{*} / \Lambda\right)^{\nu}
$$

where $\nu=\frac{\hbar}{\pi}+O\left(\hbar^{2}\right)$. The energy scale $E_{*}$ is an RG invariant (i.e., it's kept fixed with changing $\Lambda$ ), so that $\kappa \rightarrow 1$ as $\Lambda \rightarrow \infty$. Having in mind the quantization of the model, this simple analysis shows that the classical field theory (1.6) deserves special attention when $\kappa$ is close to one. 


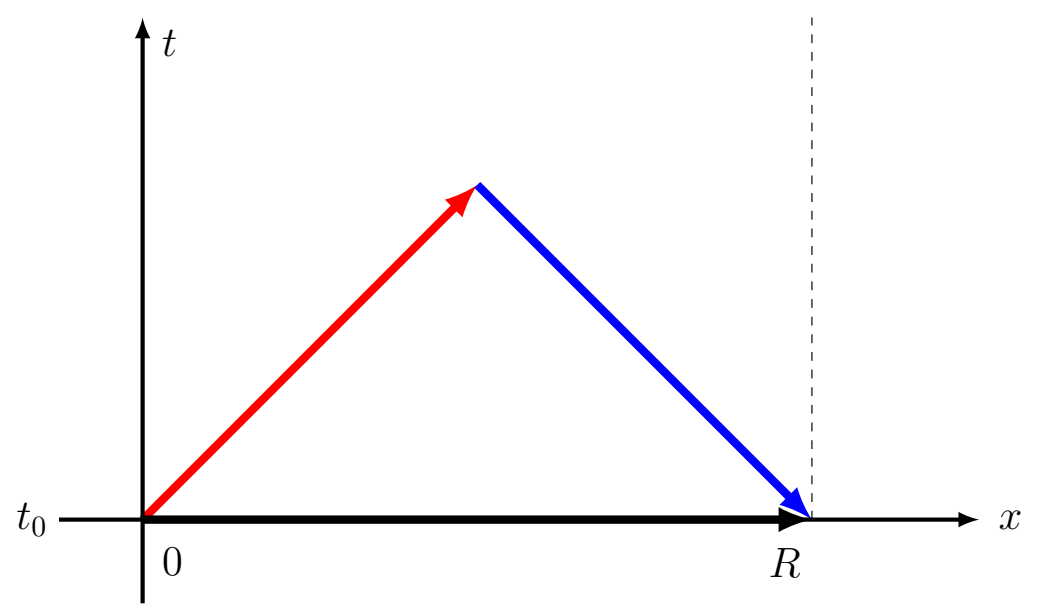

Figure 3. The integration contour along the time slice $t=t_{0}$ (black arrow) in eq (1.20) can be replaced by an integration contour along the characteristics: $x_{-}=t_{0}$ with $0<x_{+}<t_{0}+R$ (red arrow) and $x_{+}=t_{0}+R$ with $t_{0}<x_{-}<t_{0}-R$ (blue arrow).

\subsection{Wilson loops for the long sausage}

Consider the conserved charges $T_{j}(\mu)(1.20)$ for $1-\kappa \ll 1$. If the integration contour appearing in the definition (1.20) is chosen at the time slice $t=t_{0}, T_{j}(\mu)$ are expressed in terms of the pair of real variables $(\phi, \alpha)(1.17)$ and the corresponding canonically conjugate momenta. We can use the magic of the zero-curvature representation to re-express $T_{j}(\mu)$ in terms of the values of the fields $\phi(x, t)$ and $\alpha(x, t)$ on the characteristics $x_{+} \equiv t+x=t_{0}+R$ and $x_{-} \equiv t-x=t_{0}$,

$$
\begin{array}{ll}
\phi_{+}\left(x_{+}\right)=\left.\phi\right|_{x_{-}=t_{0}}, & \alpha_{+}\left(x_{+}\right)=\left.\alpha\right|_{x_{-}=t_{0}} \\
\phi_{-}\left(x_{-}\right)=\left.\phi\right|_{x_{+}=t_{0}+R}, & \alpha_{-}\left(x_{-}\right)=\left.\alpha\right|_{x_{+}=t_{0}+R} .
\end{array}
$$

Indeed, the original integration along the time slice $t=t_{0}$ in (1.20) can be replaced by the path-ordered integral over the contour glued from two light-cone segments as shown in figure 3. Denoting the light-cone values of the connection as

$$
\boldsymbol{A}_{+}\left(x_{+}\right)=\left.\boldsymbol{A}_{+}(t, x)\right|_{x_{-}=t_{0}}, \quad \boldsymbol{A}_{-}\left(x_{-}\right)=\left.\boldsymbol{A}_{-}(t, x)\right|_{x_{+}=t_{0}+R},
$$

and taking into account the boundary conditions (1.18), one can rewrite eq. (1.20) in the form

$$
T_{j} \equiv \operatorname{Tr}\left[\mathrm{e}^{-\mathrm{i} \pi k \mathrm{~h}} \boldsymbol{M}_{j}\right]=\operatorname{Tr}\left[\pi_{j}(\mathcal{M})\right]
$$

where

$$
\mathcal{M}=\mathrm{e}^{-\frac{1}{2} \mathrm{i} \alpha\left(t_{0}, R\right) \mathrm{h}} \overleftarrow{\mathcal{P}} \exp \left(\int_{t_{0}}^{t_{0}-R} \boldsymbol{A}_{-}\left(x_{-}\right) \mathrm{d} x_{-}\right) \overleftarrow{\mathcal{P}} \exp \left(\int_{t_{0}}^{t_{0}+R} \boldsymbol{A}_{+}\left(x_{+}\right) \mathrm{d} x_{+}\right) \mathrm{e}^{\frac{1}{2} \mathrm{i} \alpha\left(t_{0}, 0\right) \mathrm{h}}
$$

This formula is a convenient starting point for the quantization procedure, though one should have in mind that the light-cone fields are complicated functionals of the canonical set at $t=t_{0}$. In general, they are not independent variables and their Poisson brackets 
are not known explicitly. Fortunately, we can overcome this problem when considering the limit $\kappa \rightarrow 1$. In this limit the flat connection (1.14) simplifies considerably, since the quantities $\Pi_{+} \approx \Pi+\frac{1}{2} \partial_{x} Q^{*}, \Pi_{-} \approx \Pi^{*}-\frac{1}{2} \partial_{x} Q$ are then linearly expressed through the light-cone fields $\phi_{ \pm}$and $\alpha_{ \pm}$. It is convenient to write the constant Lie algebra elements $\boldsymbol{a}\left(\mu^{ \pm 1}\right)$ (1.12) in the form

$$
\boldsymbol{a}\left(\mu^{ \pm 1}\right)=\frac{1}{2\left(\lambda_{ \pm}-\lambda_{ \pm}^{-1}\right)}\left[\left(\lambda_{ \pm}+\lambda_{ \pm}^{-1}\right) \mathrm{h}-2 \mathrm{i}\left(\mathrm{e}_{+}+\mathrm{e}_{-}\right)\right]
$$

where the variables $\lambda_{ \pm}$are defined by the relations

$$
\lambda_{+} \lambda_{-}=\frac{1-\kappa}{1+\kappa}, \quad \lambda_{+} / \lambda_{-}=\mu^{2}
$$

and $\left(\mathrm{h}, \mathrm{e}_{+}, \mathrm{e}_{-}\right)$are the Cartan-Weyl generators (see footnote 2 on page 5 ). Then the connection component $\boldsymbol{A}_{+}\left(x_{+}\right)$only depends on the parameter $\lambda_{+}$, while $\boldsymbol{A}_{-}\left(x_{-}\right)$only depends on $\lambda_{-}$. According to (2.8) at least one of the $\left(\lambda_{+}, \lambda_{-}\right)$vanishes as $\kappa \rightarrow 1$. Let us assume that $\lambda_{-} \rightarrow 0$ and $\lambda_{+}$is kept fixed. Then, it is easy to see that

$$
\tau_{j}\left(\lambda_{+}\right) \equiv \lim _{\substack{\kappa \rightarrow 1 \\ \lambda_{+}-\text {fixed }}} T_{j}=\operatorname{Tr}\left[\boldsymbol{L}_{j}\left(\lambda_{+}\right) \mathrm{e}^{-\pi P_{1} \mathrm{~h}}\right] .
$$

Here we use the notations

$$
\boldsymbol{L}_{j}\left(\lambda_{+}\right)=\pi_{j}\left[\overleftarrow{\mathcal{P}} \exp \left\{\frac{\mathrm{i} \lambda_{+}}{1-\lambda_{+}^{2}} \int_{t_{0}}^{t_{0}+R} \mathrm{~d} x_{+}\left(V^{+} \mathrm{e}_{+}+V^{-} \mathrm{e}_{-}+\mathrm{i} \lambda_{+}\left(\partial_{+} \phi_{+}-\mathrm{i} \partial_{+} \alpha_{+}\right) \mathrm{h}\right)\right\} \mathrm{e}^{-\pi P_{1} \mathrm{~h}}\right]
$$

where

$$
V^{ \pm}=\left(\partial_{+} \phi_{+}-\mathrm{i} \partial_{+} \alpha_{+}\right) \mathrm{e}^{ \pm 2 \phi_{+}}
$$

and

$$
P_{1}=\frac{1}{2 \pi}\left(\phi_{+}\left(t_{0}+R\right)-\phi_{+}\left(t_{0}\right)\right) \text {. }
$$

Notice that the $r$-matrix in the classical Yang-Baxter Poisson algebra (1.21) depends only on the ratio of the spectral parameters and is independent of $\kappa$. Hence, we can conclude that

$$
\left\{\boldsymbol{L}_{j}\left(\lambda_{+}\right) \otimes \boldsymbol{L}_{j^{\prime}}\left(\lambda_{+}^{\prime}\right)\right\}=\left[\boldsymbol{L}_{j}\left(\lambda_{+}\right) \otimes \boldsymbol{L}_{j^{\prime}}\left(\lambda_{+}^{\prime}\right), \boldsymbol{r}_{j j^{\prime}}\left(\lambda_{+} / \lambda_{+}^{\prime}\right)\right]
$$

and also that the $\tau_{j}$ are in involution:

$$
\left\{\tau_{j}\left(\lambda_{+}\right), \tau_{j^{\prime}}\left(\lambda_{+}^{\prime}\right)\right\}=0
$$

For $1-\kappa \ll 1$ the target space of the sausage model consists of two cigars glued together, whose tips are separated by a distance $\propto \log \left(\frac{1+\kappa}{1-\kappa}\right)$ (see figure 2 ). In the $\kappa \rightarrow 1$ limit, the sausage is broken down into two disjoint half-infinite Hamilton's cigars [36]. Let us focus on one of the cigars, say the left one. The Lagrangian of the NLSM which has this target space follows from the sausage Lagrangian (1.6): one should express the sausage 
Lagrangian in terms of the fields $\phi$ and $\alpha$, perform the constant shift $\phi \rightarrow \phi+\frac{1}{2} \log \left(\frac{1-\kappa}{1+\kappa}\right)$, and finally take the limit $\kappa \rightarrow 1$. As a result one finds [37, 38]

$$
\mathcal{L}=\frac{\mathrm{e}^{2 \phi}}{2\left(1+\mathrm{e}^{2 \phi}\right)}\left(\left(\partial_{\mu} \phi\right)^{2}+\left(\partial_{\mu} \alpha\right)^{2}\right) .
$$

This suggests that the $\tau_{j}$ introduced through the limiting procedure (2.9), can be interpreted as conserved quantities in the cigar NLSM (2.15). The fields $\phi_{ \pm}$and $\alpha_{ \pm}$which appear in the construction of $\boldsymbol{L}_{j}$ (see eqs. (2.11), (2.10)), should be now understood as the light-cone values of the fields in the cigar NLSM, in the case when the field $\phi$ becomes large, i.e., in the asymptotically flat domain of the target manifold. The cigar equations of motion in this domain asymptotically approach the pair of D'Alembert equations $\partial_{\mu} \partial^{\mu} \phi \approx 0$ and $\partial_{\mu} \partial^{\mu} \alpha \approx 0$, whose general solution can be written as $\phi \approx \phi_{+}\left(x_{+}\right)+\phi_{-}\left(x_{-}\right)$and $\alpha \approx$ $\alpha_{+}\left(x_{+}\right)+\alpha_{-}\left(x_{-}\right)$. Taking the time slice $t=t_{0}$ where the field $\phi$ becomes large, one finds

$$
\begin{aligned}
& \left\{\phi_{ \pm}(x), \phi_{ \pm}^{\prime}(y)\right\}=\frac{1}{2} \delta(x-y), \quad\left\{\alpha_{ \pm}^{\prime}(x), \alpha_{ \pm}^{\prime}(y)\right\}=\frac{1}{2} \delta^{\prime}(x-y) \\
& \left\{\phi_{ \pm}^{\prime}(x), \phi_{\mp}^{\prime}(y)\right\}=\left\{\alpha_{ \pm}^{\prime}(x), \alpha_{\mp}^{\prime}(y)\right\}=\left\{\phi_{ \pm}(x), \alpha_{ \pm}^{\prime}(y)\right\}=\left\{\phi_{ \pm}(x), \alpha_{\mp}^{\prime}(y)\right\}=0,
\end{aligned}
$$

where the prime stands for the derivative w.r.t. the argument. Recall that the $\tau_{j}$ are time independent charges, and their value does not depend on the choice of $t_{0}$. Hence, we come to the conclusion that the matrix $\boldsymbol{L}_{j}(2.10)$, built from the fields $\phi_{+}(x), \alpha_{+}(x)$ satisfying the Poisson bracket relations (2.16), will obey the " $r$-matrix" Poisson bracket algebra (2.13).

The following comment is in order here. It is not difficult to see that the matrix $\boldsymbol{L}_{j}$ does not change under the constant shifts $\phi_{+} \rightarrow \phi_{+}+$const so that the Poisson bracket relations (2.16) can be lifted to

$$
\left\{\phi_{+}(x), \phi_{+}(y)\right\}=-\frac{1}{4} \epsilon(x-y),
$$

where $\epsilon(x)=2 n+1$ for $n R<x<(n+1) R ; n \in \mathbb{Z}$. The later are consistent with the quasiperiodicity condition (2.12).

\subsection{Definition and basic properties of the chiral transfer-matrices}

We may now turn to the problem of quantization. The quantum counterpart of the conserved charges (2.9) will be referred to as the chiral transfer-matrices and in what follows, will be denoted by the same symbol $\tau_{j}$. Their construction almost identically follows the steps elaborated in refs. [11, 19] in the context of the quantum KdV theory. Here we present them very briefly, referring the reader to those works for detailed explanations.

First of all we should "quantize" the Lie algebra $\mathfrak{s l}(2)$, so that $\mathrm{h}, \mathrm{e}_{ \pm}$are understood now as the generators of the quantum universal enveloping algebra $U_{q}(\mathfrak{s l}(2))$ :

$$
\left[\mathrm{h}, \mathrm{e}_{ \pm}\right]= \pm 2 \mathrm{e}_{ \pm}, \quad\left[\mathrm{e}_{+}, \mathrm{e}_{-}\right]=\frac{q^{\mathrm{h}}-q^{-\mathrm{h}}}{q-q^{-1}},
$$

where $q=\mathrm{e}^{\frac{\mathrm{i} \hbar}{2}}$. Consequently the symbol $\pi_{j}$ will stand for the $(2 j+1)$-dimensional representation of the quantum algebra. Instead of the Planck constant $\hbar$, for convenience we 
will use the parameter $n$ :

$$
\hbar \equiv \frac{2 \pi}{n}, \quad q=\mathrm{e}^{\frac{\mathrm{i} \pi}{n}} .
$$

The quantum counterpart to $\boldsymbol{L}_{j}$ is the following $(2 j+1) \times(2 j+1)$ operator valued matrix

$$
\boldsymbol{L}_{j}\left(\lambda_{+}\right)=\pi_{j}\left[\overleftarrow{\mathcal{P}} \exp \left(\mathrm{i} \lambda_{+} \int_{t_{0}}^{t_{0}+R} \mathrm{~d} x\left(V^{+} q^{\frac{\mathrm{h}}{2}} \mathbf{e}_{+}+V^{-} q^{-\frac{\mathrm{h}}{2}} \mathbf{e}_{-}\right)\right) \mathrm{e}^{-\pi P_{1} \mathrm{~h}}\right]
$$

The vertex operators $V^{ \pm}$are defined by the set of relations:

$$
V^{ \pm}(x)=\left(\frac{1}{2} c^{ \pm} \partial_{x}-\mathrm{i} \frac{\sqrt{n+2}}{n} \vartheta_{+}^{\prime}(x)\right) \mathrm{e}^{ \pm \frac{2 \varphi_{+}}{\sqrt{n}}}(x),
$$

where $c^{ \pm}$are some constants and

$$
\begin{aligned}
& \varphi_{+}(x)=Q_{1}+\frac{2 \pi x}{R} \sqrt{n} P_{1}+\mathrm{i} \sum_{m \neq 0} \frac{a_{m}}{m} \mathrm{e}^{-\frac{2 \pi \mathrm{i} m}{R} x} \\
& \vartheta_{+}(x)=Q_{2}+\frac{2 \pi x}{R} \sqrt{n+2} P_{2}+\mathrm{i} \sum_{m \neq 0} \frac{b_{m}}{m} \mathrm{e}^{-\frac{2 \pi \mathrm{i} m}{R} x},
\end{aligned}
$$

with

$$
\left[a_{m}, a_{l}\right]=\left[b_{m}, b_{l}\right]=\frac{m}{2} \delta_{m+l, 0}, \quad\left[Q_{1}, \sqrt{n} P_{1}\right]=\left[Q_{2}, \sqrt{n+2} P_{2}\right]=\frac{\mathrm{i}}{2} .
$$

Let $\mathcal{F}_{p_{1}, p_{2}} \equiv \mathcal{F}_{\mathbf{p}}$ ("Fock space") be the highest weight module of the Heisenberg algebra (2.23) with the highest weight vector $|\mathbf{p}\rangle$ defined by the equations

$$
P_{1}|\mathbf{p}\rangle=\frac{p_{1}}{n}|\mathbf{p}\rangle, \quad P_{2}|\mathbf{p}\rangle=\frac{p_{2}}{n+2}|\mathbf{p}\rangle .
$$

It is easy to see that

$$
V^{ \pm}(x): \quad \mathcal{F}_{p_{1}, p_{2}} \mapsto \mathcal{F}_{p_{1} \mp \mathrm{i}, p_{2}},
$$

and therefore the matrix elements of $\boldsymbol{L}_{j}(\lambda)$ are operators in $\oplus_{m=-\infty}^{\infty} \mathcal{F}_{p_{1}+\mathrm{i} m, p_{2}}$. The expression (2.20) contains the ordered exponential which can be formally written in terms of a power series in $\lambda$ as

$$
\boldsymbol{L}_{j}\left(\lambda_{+}\right)=\pi_{j}\left[\sum_{m=0}^{\infty}\left(\mathrm{i} \lambda_{+}\right)^{m} \int_{t_{0}+R>x_{m}>\ldots x_{1}>t_{0}} \mathrm{~d} x_{m} \cdots \mathrm{d} x_{1} \boldsymbol{K}\left(x_{m}\right) \cdots \boldsymbol{K}\left(x_{1}\right) \mathrm{e}^{-\pi P_{1} \mathrm{~h}}\right],
$$

where

$$
\boldsymbol{K}(x)=V^{+} q^{\frac{\mathrm{h}}{2}} \mathbf{e}_{+}+V^{-} q^{-\frac{\mathrm{h}}{2}} \mathbf{e}_{-} .
$$

However, since

$$
\left.V^{ \pm}\left(x_{2}\right) V^{\mp}\left(x_{1}\right)\right|_{x_{2} \rightarrow x_{1}+0} \sim \frac{a}{2 n^{2}} q^{-1}\left(x_{2}-x_{1}\right)^{-2\left(1-\frac{1}{n}\right)},
$$

where $a=n+2+(n-2) c^{+} c^{-}$, the integrals in (2.26) diverge. As explained in [19], the commutation relations

$$
V^{\sigma_{1}}\left(x_{1}\right) V^{\sigma_{2}}\left(x_{2}\right)=q^{2 \sigma_{1} \sigma_{2}} V^{\sigma_{2}}\left(x_{2}\right) V^{\sigma_{1}}\left(x_{1}\right), \quad x_{2}>x_{1} \quad\left(\sigma_{1,2}= \pm\right)
$$


allow one to re-express the integrals in (2.26) in terms of two basic contour integrals

$$
\mathcal{X}_{0}=\frac{1}{q-q^{-1}} \int_{t_{0}}^{t_{0}+R} \mathrm{~d} x V^{-}(x), \quad \mathcal{X}_{1}=\frac{1}{q-q^{-1}} \int_{t_{0}}^{t_{0}+R} \mathrm{~d} x V^{+}(x) .
$$

This procedure yields an unambiguous definition of the ordered exponential in (2.20) for $n \neq 2,4,6 \ldots$ The case of even $n$ needs some special attention and we will return to it later.

Notice that the above analytical regularization of the $\mathcal{P}$-ordered exponential (2.20) is not applicable at $n \rightarrow \infty$. This limit can be studied within the renormalized perturbation theory in $\hbar=\frac{2 \pi}{n}$. As usual, the perturbative expansion will involve the counterterms depending on the UV regulator. At $\hbar=0$ the counterterms give rise to anomalous contributions which remain finite in the limit where the UV regulator goes away. This makes the classical limit not entirely straightforward, however if the anomalous terms are properly taken into account, the formula (2.20) precisely reduces to its classical version (2.10) in the limit $\hbar \rightarrow 0$. The relevant calculations will be presented elsewhere.

The operator valued matrices $\boldsymbol{L}_{j}$ (2.20) are designed in such a way that, for arbitrary chosen constants $c^{ \pm}$and $t_{0}$, they obey the quantum Yang-Baxter algebra

$$
\boldsymbol{R}_{j j^{\prime}}\left(\lambda_{+}^{\prime} / \lambda_{+}\right)\left(\boldsymbol{L}\left(\lambda_{+}\right) \otimes 1\right)\left(1 \otimes \boldsymbol{L}\left(\lambda_{+}^{\prime}\right)\right)=\left(1 \otimes \boldsymbol{L}\left(\lambda_{+}^{\prime}\right)\right)\left(\boldsymbol{L}\left(\lambda_{+}\right) \otimes 1\right) \boldsymbol{R}_{j j^{\prime}}\left(\lambda_{+}^{\prime} / \lambda_{+}\right),
$$

where the matrix $\boldsymbol{R}_{j j^{\prime}}(\lambda)$ is the trigonometric solution to the Yang-Baxter equation which acts in the space $\pi_{j} \otimes \pi_{j^{\prime}}$. In particular

$$
\boldsymbol{R}_{\frac{1}{2} \frac{1}{2}}(\lambda)=\left(\begin{array}{cccc}
q^{-1} \lambda-q \lambda^{-1} & 0 & 0 & 0 \\
0 & \lambda-\lambda^{-1} & q^{-1}-q & 0 \\
0 & q^{-1}-q & \lambda-\lambda^{-1} & 0 \\
0 & 0 & 0 & q^{-1} \lambda-q \lambda^{-1}
\end{array}\right)
$$

The proof of eq. (2.31) follows that from the work [19].

The chiral transfer-matrices, defined similar to (2.9),

$$
\tau_{j}\left(\lambda_{+}\right)=\operatorname{Tr}\left[\boldsymbol{L}_{j}\left(\lambda_{+}\right) \mathrm{e}^{-\pi P_{1} \mathrm{~h}}\right]
$$

satisfy the commutativity condition

$$
\left[\tau_{j}\left(\lambda_{+}\right), \tau_{j^{\prime}}\left(\lambda_{+}^{\prime}\right)\right]=0,
$$

as a simple consequence of (2.31). Notice that the chiral transfer-matrices act inside a single Fock space, whereas the same is not true for an arbitrary element of $\boldsymbol{L}_{j}(\lambda)$. Furthermore, the Fock space $\mathcal{F}_{\mathbf{p}}$ naturally splits into the finite dimensional "level subspaces"

$$
\mathcal{F}_{\mathbf{p}}=\oplus_{L=0}^{\infty} \mathcal{F}_{\mathbf{p}}^{(L)}: \quad \mathbb{L} \mathcal{F}_{\mathbf{p}}^{(L)}=L \mathcal{F}_{\mathbf{p}}^{(L)},
$$

where the grading operator is given by

$$
\mathbb{L}=2 \sum_{m=1}^{\infty}\left(a_{-m} a_{m}+b_{-m} b_{m}\right) .
$$


Using the relation,

$$
V^{ \pm}(x+R)=q^{2} \mathrm{e}^{ \pm 4 \pi P_{1}} V^{ \pm}(x)
$$

one can show (see appendix $\mathrm{C}$ from [19]) that the $\tau_{j}\left(\lambda_{+}\right)$commute with the grading operator, and therefore, act invariantly in each finite-dimensional level subspace:

$$
\tau_{j}\left(\lambda_{+}\right): \mathcal{F}_{\mathbf{p}}^{(L)} \mapsto \mathcal{F}_{\mathbf{p}}^{(L)} .
$$

The Fock space $\mathcal{F}_{\mathbf{p}}$ can be equipped with an inner product consistent with the Hermiticity conditions $a_{m}^{\dagger}=a_{-m}, b_{m}^{\dagger}=b_{-m}$ imposed on the Heisenberg operators (2.23). It is not difficult to show that for real $p_{1}^{2}, p_{2}^{2}$ and $\lambda_{+}^{2}, \tau\left(\lambda_{+}\right)$is a Hermitian operator and

$$
\left[\tau\left(\lambda_{+}\right)\right]^{\dagger}=\tau\left( \pm \lambda_{+}^{*}\right)
$$

Notice that the commutativity with the grading operators can be interpreted as the independence of the chiral transfer-matrix on the arbitrary chosen constant $t_{0}$. It turns out that they further do not depend on the constants $c^{ \pm}$appearing in the definition of the vertex operators $V^{ \pm}(2.21)$. Also, a simple dimensional analysis shows that the spectral parameter $\lambda_{+}$and $R$ occur in the chiral transfer-matrix through the combination $\lambda_{+}^{2} R^{\frac{2}{n}}$ only. It is convenient to introduce a dimensionless spectral parameter $\lambda$ by means of the relation

$$
\lambda^{2}=\Gamma^{2}\left(1+\frac{1}{n}\right)\left(\frac{n R}{2 \pi}\right)^{\frac{2}{n}} \lambda_{+}^{2}
$$

and treat the chiral transfer-matrices as functions of this variable rather than the dimensionful $\lambda_{+}$.

The chiral transfer-matrices are not independent operators for different values of $j=$ $\frac{1}{2}, 1, \ldots$. They can be expressed through the "fundamental" transfer-matrix $\tau_{\frac{1}{2}}(\lambda)$ by the so-called fusion relation [39-41]

$$
\tau_{j}\left(\lambda q^{j+\frac{1}{2}}\right) \tau_{\frac{1}{2}}(\lambda)=\tau_{j+\frac{1}{2}}\left(\lambda q^{j}\right)+\tau_{j-\frac{1}{2}}\left(\lambda q^{j+1}\right),
$$

supplemented by the condition $\tau_{0}=1$. In what follows, we will mostly focus on the fundamental transfer-matrix and use the notation $\tau \equiv \tau_{\frac{1}{2}}$. The integrable structures associated with the commuting family of operators $\tau_{j}(\lambda)$ were already studied in the context of the so-called paperclip model - an integrable model with boundary interaction [43]. Here for convenience we make a short summary of some basic properties of the operator $\tau(\lambda)$.

For arbitrary complex $\mathbf{p}=\left(p_{1}, p_{2}\right)$, the operator $\tau(\lambda) \in \operatorname{End}\left(\mathcal{F}_{\mathbf{p}}\right)$ is an entire function of $\lambda^{2}$ in the sense that all its matrix elements and eigenvalues are entire functions of this variable. Thus the power series

$$
\tau(\lambda)=2 \cosh \left(\frac{2 \pi p_{1}}{n}\right)+\sum_{m=1}^{\infty} \mathfrak{t}_{m} \lambda^{2 m}
$$

converges in the whole complex plane of $\lambda^{2}$ and defines an entire function with an essential singularity at $\lambda^{2}=\infty$. The asymptotic expansion near the essential singularity is of primary interest. It can be written as

$$
\tau(\lambda)=\exp \left(\frac{2 \pi}{\sin \left(\frac{\pi n}{2}\right)}\left(-\lambda^{2}\right)^{\frac{n}{2}}\right) \tilde{\tau}\left(\mathrm{i}\left(-\lambda^{2}\right)^{-\frac{n}{2(n+2)}}\right)
$$


where $\tilde{\tau}$ is a formal power series of the form

$$
\tilde{\tau}(\tilde{\lambda}) \asymp 2 \cos \left(\frac{2 \pi p_{2}}{n+2}\right)+\sum_{m=1}^{\infty} \tilde{\mathfrak{t}}_{m} \tilde{\lambda}^{2 m} .
$$

This asymptotic expansion can be applied for arbitrary complex $\mathbf{p}=\left(p_{1}, p_{2}\right)$ and $n \neq$ $2,4,6 \ldots$ Furthermore, in the case $n \geq 1$ it holds true for $\left|\arg \left(-\lambda^{2}\right)\right|<\pi$.

The expansion coefficients in (2.42) and (2.44) form two infinite sets of mutually commuting operators. Using the terminology of the work [18], we will refer to $\left\{\mathfrak{t}_{m}\right\}_{m=1}^{\infty}$ and $\left\{\tilde{\mathfrak{t}}_{m}\right\}_{m=1}^{\infty}$ as the nonlocal and dual nonlocal Integrals of Motions (IM), respectively. Remarkably, the formal power series $\tilde{\tau}(\tilde{\lambda})$ can be written in a form similar to (2.33). Namely [43],

$$
\tilde{\tau}(\tilde{\lambda})=\operatorname{Tr}\left[\overleftarrow{\mathcal{P}} \exp \left(\mathrm{i} \tilde{\lambda}_{+} \int_{t_{0}}^{t_{0}+R} \mathrm{~d} x\left(\Psi^{+} \sigma_{+}+\Psi^{-} \sigma_{-}\right)\right) \mathrm{e}^{-2 \pi \mathrm{i} P_{2} \sigma_{3}}\right]
$$

where $\sigma_{3}, \sigma_{ \pm}=\frac{1}{2}\left(\sigma_{1} \pm \mathrm{i} \sigma_{2}\right)$ are the conventional Pauli matrices and the vertex operators $\Psi^{ \pm}$are given by

$$
\Psi^{ \pm}(x)=\left(\frac{\sqrt{n}}{n+2} \varphi_{+}^{\prime}(x)+\frac{1}{2} \partial_{x}\right) \mathrm{e}^{ \pm \frac{2 \mathrm{i} \theta_{+}}{\sqrt{n+2}}}(x) .
$$

The scale dimension of $\Psi^{ \pm}$is equal to $1+\frac{1}{n+2}$ and we assume here that they are normalized in such a way that

$$
\left.\Psi^{ \pm}\left(x_{2}\right) \Psi^{\mp}\left(x_{1}\right)\right|_{x_{2} \rightarrow x_{1}+0} \sim \frac{2}{(n+2)^{2}}\left(x_{2}-x_{1}\right)^{-2\left(1+\frac{1}{n+2}\right)} .
$$

Because of the divergencies, the path ordered exponential in (2.45) should be understood in the same manner as (2.20), i.e., the formal expansion in a power series of $\tilde{\lambda}_{+}$should be rewritten in terms of the basic contour integrals similar to (2.30). With this analytical regularization the r.h.s. of eq. (2.45) becomes a formal power series in $\tilde{\lambda}_{+}^{2} R^{-\frac{2}{n+2}}$ with unambiguously defined expansion coefficients. Up to a factor similar to that in (2.40), this combination can be identified with $\tilde{\lambda}^{2}$ in eq. (2.44):

$$
\tilde{\lambda}^{2}=\Gamma^{2}\left(1-\frac{1}{n+2}\right)\left(\frac{(n+2) R}{2 \pi}\right)^{-\frac{2}{n+2}} \tilde{\lambda}_{+}^{2} .
$$

For future reference we present here explicit formulae for the "vacuum" eigenvalues of the operators $\mathfrak{t}_{1}$ and $\tilde{\mathfrak{t}}_{1}$ corresponding to the highest weight vector $|\mathbf{p}\rangle \in \mathcal{F}_{\mathbf{p}}(2.24)$ :

$$
t_{1}\left(p_{1}, p_{2}\right)=\left(\frac{2}{n}\right)^{\frac{2}{n}} \frac{\Gamma\left(\frac{1}{2}+\frac{1}{n}\right)}{\sqrt{\pi} \Gamma\left(1+\frac{1}{n}\right)}\left(\frac{n+2}{n-2}+\frac{4 p_{2}^{2}}{1+4 p_{1}^{2}}\right) \frac{\pi^{2}}{\Gamma\left(\frac{1+2 \mathrm{i} p_{1}}{n}\right) \Gamma\left(\frac{1-2 \mathrm{i} p_{1}}{n}\right)}
$$

and

$$
\tilde{t}_{1}\left(p_{1}, p_{2}\right)=\left(\frac{n+2}{2}\right)^{\frac{2}{n+2}} \frac{\Gamma\left(\frac{1}{2}-\frac{1}{n+2}\right)}{\sqrt{\pi} \Gamma\left(1-\frac{1}{n+2}\right)}\left(\frac{n}{n+4}-\frac{4 p_{1}^{2}}{1-4 p_{2}^{2}}\right) \frac{\pi^{2}}{\Gamma\left(-\frac{1-2 p_{2}}{n+2}\right) \Gamma\left(-\frac{1+2 p_{2}}{n+2}\right)} .
$$


An efficient integral representation for calculating the vacuum eigenvalue $\tilde{t}_{2}\left(p_{1}, p_{2}\right)$ can be found in appendix A of ref. [43].

For even $n$, the chiral transfer-matrices require some careful handling. In this case $\tau(\lambda)$ can be defined through the limiting procedure

$$
\left.\tau(\lambda)\right|_{n=2 l}=\left.\lim _{\epsilon \rightarrow 0} \exp \left(-\frac{4}{\epsilon} \lambda^{2 l}\right) \tau(\lambda)\right|_{n=2 l+\epsilon} \quad(l=1,2,3 \ldots),
$$

so that the asymptotic formula (2.43) should be substituted by

$$
\left.\tau(\lambda)\right|_{n=2 l}=\left.\exp \left(2 \lambda^{n} \log \left(-\lambda^{2}\right)\right) \tilde{\tau}\left(\mathrm{i}\left(-\lambda^{2}\right)^{-\frac{l}{2(l+1)}}\right)\right|_{n=2 l} .
$$

The formulae (2.43), (2.44) are not valid for positive real $\lambda^{2}$. In order to describe the asymptotic behaviour for $\lambda^{2} \rightarrow+\infty$, it is convenient to substitute the set of dual nonlocal IM (2.44) by the set $\left\{\tilde{\mathfrak{g}}_{m}\right\}_{m=1}^{\infty}$ which are algebraically expressed in terms of the former through the relation

$$
2 \cos \left(2 \pi P_{2}\right)+\sum_{m=1}^{\infty} \tilde{\mathfrak{t}}_{m} z^{m}=2 \cos \left(2 \pi P_{2}\right) \exp \left(\sum_{m=1}^{\infty} \tilde{\mathfrak{g}}_{m} z^{m}\right) .
$$

Then, for arbitrary complex $\mathbf{p}=\left(p_{1}, p_{2}\right), n \neq 2,4,6 \ldots, n \geq 1$,

$$
\tau(\lambda)=4 \cos \left(\frac{2 \pi p_{2}}{n+2}\right) \mathrm{e}^{H\left(\lambda^{2}\right)} \cos \left(G\left(\lambda^{2}\right)\right) \quad \text { as } \quad \lambda^{2} \rightarrow+\infty,
$$

where

$$
\begin{aligned}
& H(z) \asymp 2 \pi \cot \left(\frac{\pi n}{2}\right) z^{\frac{n}{2}}+\sum_{m=1}^{\infty} \tilde{\mathfrak{g}}_{m} \cos \left(\frac{2 \pi m}{n+2}\right) z^{-\frac{n m}{n+2}}+O\left(z^{-\infty}\right) \\
& G(z) \asymp 2 \pi z^{\frac{n}{2}}+\sum_{m=1}^{\infty} \tilde{\mathfrak{g}}_{m} \sin \left(\frac{2 \pi m}{n+2}\right) z^{-\frac{n m}{n+2}}+O\left(z^{-\infty}\right) .
\end{aligned}
$$

For even $n$, the first term in the formal power series $H(z)$ should be replaced by $2 z^{\frac{n}{2}} \log (z)$.

\subsection{Basic facts about the quantum cigar}

In the previous subsection, we described the formal algebraic construction of the chiral transfer-matrices. Here we briefly discuss how $\tau_{j}(\lambda)$ can be understood as operators in the quantum cigar NLSM (for more details on the quantum cigar see, e.g., ref. [44]).

The cigar NLSM was introduced before at the classical level by means of the Lagrangian (2.15). In the classical field theory, it is natural to consider the following scattering problem. Suppose that at $t \rightarrow-\infty$ we are given the field configuration within the asymptotically flat domain of the target manifold, i.e.,

$$
\begin{aligned}
& \left.\phi(t, x)\right|_{t \rightarrow-\infty} \asymp \phi_{0}^{(\mathrm{in})}+\frac{4 \pi}{R} P_{1}^{(\mathrm{in})} t+\sum_{m \neq 0} \frac{\mathrm{i}}{m}\left(a_{m}^{(\mathrm{in})} \mathrm{e}^{-\frac{2 \pi \mathrm{i} m}{R}(t+x)}+\bar{a}_{m}^{(\mathrm{in})} \mathrm{e}^{-\frac{2 \pi \mathrm{i} m}{R}(t-x)}\right)(2.5 \\
& \left.\alpha(t, x)\right|_{t \rightarrow-\infty} \asymp \alpha_{0}^{(\mathrm{in})}+\frac{2 \pi}{R}(k x+\tilde{k} t)+\sum_{m \neq 0} \frac{\mathrm{i}}{m}\left(b_{m}^{(\mathrm{in})} \mathrm{e}^{-\frac{2 \pi \mathrm{i} m}{R}(t+x)}+\bar{b}_{m}^{(\mathrm{in})} \mathrm{e}^{-\frac{2 \pi \mathrm{i} m}{R}(t-x)}\right) .
\end{aligned}
$$




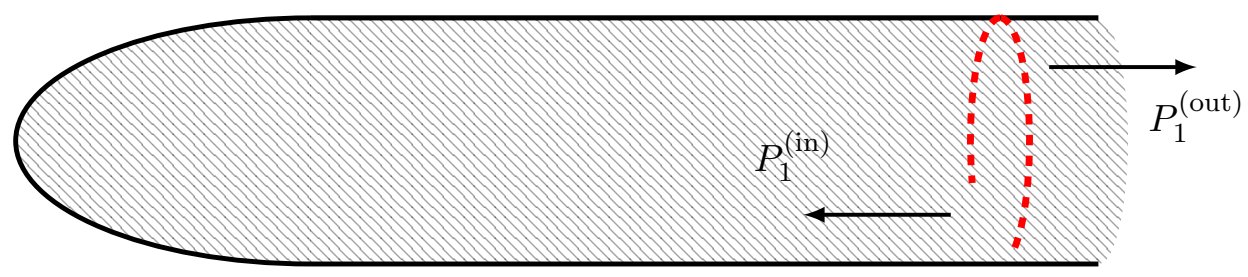

Figure 4. The classical scattering problem in the cigar NLSM. From the asymptotically flat domain the string approaches the tip, scatters and then escapes back to the flat region. After the scattering process the zero mode momentum changes sign.

In writing this equation, we took into account the boundary conditions (1.18). Also, the constant $\tilde{k}$ is the conserved charge for the Noether $\mathrm{U}(1)$-current associated with the Lagrangian (2.15). The set, $\mathcal{A}^{(\mathrm{in})}=\left\{\phi_{0}^{(\mathrm{in})}, P_{1}^{(\mathrm{in})}, \alpha_{0}^{(\mathrm{in})}, \tilde{k}, a_{m}^{(\mathrm{in})}, b_{m}^{(\mathrm{in})}\right\}$, can be interpreted as a classical "in-state" for a string propagating on the target manifold (see figure 4). The nontrivial interaction occurs at some finite time when the fields take values in the vicinity of the tip of the cigar. After scattering at the tip, as $t \rightarrow+\infty$, the field configuration returns to the asymptotically flat domain and takes the same form as in the r.h.s. of (2.55) with the in-state $\mathcal{A}^{\text {(in) }}$ replaced by the out-state $\mathcal{A}^{\text {(out) }}=\left\{\phi_{0}^{\text {(out) }}, P_{1}^{\text {(out) }}, \alpha_{0}^{\text {(out) }}, \tilde{k}, a_{m}^{\text {(out) }}, b_{m}^{\text {(out) }}\right\}^{3}$ The classical scattering problem can be formulated as the problem of finding the canonical transformation which maps $\mathcal{A}^{\text {(in) }}$ to $\mathcal{A}^{\text {(out) }}$. It turns out that the theory possesses two infinite sets of left- and right-currents [45], i.e.,

$$
\partial_{-} W_{s}=0, \quad \partial_{+} \bar{W}_{s}=0, \quad(s=2,3, \ldots),
$$

so that the classical dynamics of the fields are strongly constrained. In particular, the magnitude of the zero-mode momentum remains unchanged after the scattering (see figure 4),

$$
P_{1}^{(\text {out })}=-P_{1}^{(\text {in })} .
$$

Consider now the quantum theory. First of all we note that the value of the U(1) charge is quantized so that $(n+2) \tilde{k}=m \in \mathbb{Z}$. Thus the space of states of the quantum theory splits into orthogonal subspaces $\mathcal{H}_{k, m}$ labeled by the twist parameter $k$ and the integer $m$. The quantum theory still possesses the chiral currents satisfying eqs. (2.56). As a result, $\mathcal{H}_{k, m}$ can be decomposed into the highest weight irreps of the $W$-algebra, $\mathfrak{W} \otimes \overline{\mathfrak{W}}$ generated by the fields $W_{s}$ and $\bar{W}_{s}$ [45]. Let $\mathcal{V}_{h}\left(\overline{\mathcal{V}}_{\bar{h}}\right)$ be the highest weight representation of the chiral $W$-algebra $\mathfrak{W}(\overline{\mathfrak{W}})$ labeled by the highest weight $h(\bar{h})$. Then, schematically,

$$
\mathcal{H}_{k, m}=\underset{\{h, \bar{h}\}}{\oplus} \mathcal{V}_{h} \otimes \overline{\mathcal{V}}_{\bar{h}}
$$

The highest weight $h$ can be chosen to be a pair of numbers $(\Delta, w)$, where $\Delta$ coincides with the conformal dimensions of the highest weight vector, while $w$ is the eigenvalue of

\footnotetext{
${ }^{3}$ Strictly speaking, the winding number $k$ is only conserved modulo an integer, i.e., $k^{(\text {out })}-k^{(\text {in })} \in \mathbb{Z}$. Here we ignore this and assume that $k^{(\text {out })}=k^{(\text {in })} \in\left(-\frac{1}{2},+\frac{1}{2}\right]$.
} 
the dimensionless conserved charge $R^{2} \int_{0}^{R} \mathrm{~d} x W_{3}(x)$, and similar for $\bar{h}$. Let us first focus on the "left" component $\mathcal{V}_{h}$ in the tensor product $\mathcal{V}_{h} \otimes \overline{\mathcal{V}}_{\bar{h}}$.

It should be clear that the quantum counterpart to the left components of the inasymptotic fields (2.55) can be identified with the fields $\varphi_{+}$and $\vartheta_{+}$given by (2.22). Since the quantum fields $W_{s}$ are chiral currents, i.e. $W_{s}(t, x)=W_{s}(t+x)$, they can be expressed in terms of the asymptotic fields $\varphi_{+}$and $\vartheta_{+}$. Indeed, for given $s, W_{s}$ is a certain order- $s$ homogeneous polynomial with constant coefficients w.r.t. the fields $\varphi_{+}^{\prime}, \vartheta_{+}^{\prime}$ and their higher derivatives (in other words, any monomials appearing within $W_{s}$ contains exactly $s$ derivative symbols). This implies that the Fock space $\mathcal{F}_{\mathbf{p}}$, which is the space of representation for the fields $\varphi_{+}^{\prime}, \vartheta_{+}^{\prime}$, possesses the structure of the highest weight representation of the chiral $W$-algebra. It turns out that for real $\mathbf{p}$, the Fock space $\mathcal{F}_{\mathbf{p}}$ coincides with irrep $\mathcal{V}_{h}$ as a linear space, provided that $h=(\Delta, w)$ is related to $\mathbf{p}=\left(p_{1}, p_{2}\right)$ as follows

$$
\begin{aligned}
& \Delta\left(p_{1}, p_{2}\right)=\frac{p_{1}^{2}}{n}+\frac{p_{2}^{2}}{n+2}+\frac{1}{4 n} \\
& w\left(p_{1}, p_{2}\right)=p_{2}\left(p_{1}^{2}+\frac{3 n+2}{3(n+2)} p_{2}^{2}-\frac{2 n+1}{12}\right) .
\end{aligned}
$$

In fact, one can use these formulae to conveniently parameterize the highest weight $h$ by the pair $\left(p_{1}, p_{2}\right): \mathcal{V}_{h} \equiv \mathcal{V}_{p_{1}, p_{2}}$. With this notation, a more accurate version of eq. (2.58) reads as

$$
\mathcal{H}_{k, m}=\int_{p_{1}<0}^{\oplus} \mathcal{V}_{p_{1}, p_{2}} \otimes \overline{\mathcal{V}}_{p_{1}, \bar{p}_{2}},
$$

where

$$
p_{2}=\frac{1}{2}(m+(n+2) k), \quad \bar{p}_{2}=\frac{1}{2}(m-(n+2) k) .
$$

The direct integral in (2.60) does not include the domain with positive $p_{1}$, since, as follows from eqs. (2.59), $\mathcal{V}_{p_{1}, p_{2}} \equiv \mathcal{V}_{-p_{1}, p_{2}}$.

A basis of in-asymptotic states in $\mathcal{H}_{k, m}$ is formed by

$$
a_{-m_{1}}^{(\mathrm{in})} \ldots a_{-m_{N}}^{(\mathrm{in})} \bar{a}_{-\bar{m}_{1}}^{(\mathrm{in})} \ldots \bar{a}_{-\bar{m}_{\bar{N}}}^{(\mathrm{in})} b_{-m_{1}}^{(\mathrm{in})} \ldots b_{-m_{M}}^{(\mathrm{in})} \bar{b}_{-\bar{m}_{1}}^{(\mathrm{in})} \ldots \bar{b}_{-\bar{m}_{\bar{M}}}^{(\mathrm{in})}|\operatorname{vac}\rangle
$$

and can be identified with the states from the tensor product of the Fock space $\mathcal{F}_{p_{1}, p_{2}} \otimes$ $\overline{\mathcal{F}}_{p_{1}, \bar{p}_{2}}$ :

$$
a_{-m_{1}} \ldots a_{-m_{N}} \bar{a}_{-\bar{m}_{1}} \ldots \bar{a}_{-\bar{m}_{\bar{N}}} b_{-m_{1}} \ldots b_{-m_{M}} \bar{b}_{-\bar{m}_{1}} \ldots \bar{b}_{-\bar{m}_{\bar{M}}}\left|p_{1}, p_{2}\right\rangle \otimes\left|p_{1}, \bar{p}_{2}\right\rangle .
$$

Similarly for the out-states, one has

$$
\begin{aligned}
& \left.a_{-m_{1}}^{\text {(out) }} \ldots a_{-m_{N}}^{\text {(out) }} \bar{a}_{-\bar{m}_{1}}^{\text {(out) }} \ldots \bar{a}_{-\bar{m}_{\bar{N}}}^{\text {(out) }} b_{-m_{1}}^{\text {(out) }} \ldots b_{-m_{M}}^{\text {(out) }} \bar{b}_{-\bar{m}_{1}}^{\text {(out) }} \ldots \bar{b}_{-\bar{m}_{\bar{M}}}^{\text {(out) }} \mid \text { vac }\right\rangle \sim \\
& \sim a_{-m_{1}} \ldots a_{-m_{N}} \bar{a}_{-\bar{m}_{1}} \ldots \bar{a}_{-\bar{m}_{\bar{N}}} b_{-m_{1}} \ldots b_{-m_{M}} \bar{b}_{-\bar{m}_{1}} \ldots \bar{b}_{-\bar{m}_{\bar{M}}}\left|-p_{1}, p_{2}\right\rangle \otimes\left|-p_{1}, \bar{p}_{2}\right\rangle .
\end{aligned}
$$

Usually, the $S$-matrix is introduced as a unitary operator which relates the in- and outasymptotic bases. In the case under consideration, the $S$-matrix can be interpreted as the intertwiner acting between the Fock spaces:

$$
\hat{S}: \quad \mathcal{F}_{p_{1}, p_{2}} \otimes \overline{\mathcal{F}}_{p_{1}, \bar{p}_{2}} \mapsto \mathcal{F}_{-p_{1}, p_{2}} \otimes \overline{\mathcal{F}}_{-p_{1}, \bar{p}_{2}} .
$$


It turns out that the operator $\hat{S}$ has the following structure

$$
\hat{S}=S_{0}(\mathbf{p}) \hat{S}_{L} \otimes \hat{S}_{R}
$$

where $\hat{S}_{L}$ intertwines the level subspaces, $\hat{S}_{L}: \mathcal{F}_{p_{1}, p_{2}}^{(L)} \mapsto \mathcal{F}_{-p_{1}, p_{2}}^{(L)}$, and is normalized by the condition $\hat{S}_{L}\left|p_{1}, p_{2}\right\rangle=\left|-p_{1}, p_{2}\right\rangle$, and similarly for $\hat{S}_{R}$. For a given level $\ell$, the construction of the operators $\hat{S}_{L, R}$ is a straightforward algebraic task. The more delicate problem is finding the overall scalar factor $S_{0}(\mathbf{p})$. It was obtained in the minisuperspace approximation in ref. [46]. The exact form of $S_{0}(\mathbf{p})$ has been known since the unpublished work of the Zamolodchikov brothers [47]. ${ }^{4}$

Returning to the chiral transfer-matrices, let us note that these operators should act in the Hilbert space of the quantum cigar, and therefore their action should commute with the intertwiner $\hat{S}:^{5}$

$$
\hat{S} \tau(\lambda)=\tau(\lambda) \hat{S}
$$

In practice, this condition implies that all matrix elements of the (dual) nonlocal IM in the basis of Fock states (2.63) are even functions of $p_{1}$ (for illustration see eqs. (2.48), (2.49)).

The quantum cigar also possesses an infinite set of the so-called local IM acting in $\mathcal{H}_{k, m}$. To get some feeling for these operators, we need to remind ourselves of an important feature of the model. Namely, it admits an equivalent "dual" description in terms of the so-called sine-Liouville model. The dual Lagrangian is given by [47]

$$
\mathcal{L}^{(\text {dual })}=\frac{1}{4 \pi}\left(\left(\partial_{\sigma} \varphi\right)^{2}+\left(\partial_{\sigma} \vartheta\right)^{2}\right)+2 \mathcal{M} \mathrm{e}^{-\sqrt{n} \varphi} \cos (\sqrt{n+2} \vartheta)
$$

with the sine-Liouville fields satisfying the boundary conditions

$$
\varphi(t, x+R)=\varphi(t, x), \quad \vartheta(t, x+R)=\vartheta(t, x)+\frac{2 \pi m}{\sqrt{n+2}} .
$$

Notice that the "coupling" $\mathcal{M}$ is a somewhat fake parameter of the Lagrangian — by an additive shift $\varphi \mapsto \varphi+$ const the value of $\mathcal{M}$ can be chosen to be any real number. Nevertheless, it is convenient to keep it unspecified.

To understand the relation between the fields in the NLSM and its dual description, let us take the "zero-mode" of the field $\varphi$

$$
\varphi_{0}=\int_{0}^{R} \frac{\mathrm{d} x}{R} \varphi(x)
$$

and consider the region $\varphi_{0} \rightarrow+\infty$ in configuration space. In this asymptotic domain, the potential term in the action (2.68) can be neglected and $\frac{\varphi}{\sqrt{n}} \asymp \phi+$ const, while $\frac{\vartheta}{\sqrt{n+2}}$ can be identified with $\tilde{\alpha}$ - the field from the cigar NLSM defined by the relation $J_{\mu}=\epsilon_{\mu \nu} \partial_{\nu} \tilde{\alpha}$, where $J_{\mu}$ stands for the Noether U(1)-current.

\footnotetext{
${ }^{4}$ Although Zamolodchikov's notes have never been published, they were broadly distributed within the scientific community.

${ }^{5}$ The intertwiner $\hat{S}$ should not to be confused with the so called "reflection" operator $\hat{R}: \mathcal{F}_{p_{1}, p_{2}} \otimes$ $\overline{\mathcal{F}}_{p_{1}, \bar{p}_{2}} \mapsto \mathcal{F}_{p_{1}, p_{2}} \otimes \overline{\mathcal{F}}_{p_{1}, \bar{p}_{2}}$, and $[\hat{R}, \tau(\lambda)]=0$. Note that $\hat{R}=\hat{\sigma} \circ \hat{S}$ where $\hat{\sigma}=\hat{\sigma}_{L} \otimes \hat{\sigma}_{R}$ and the chiral intertwiners $\hat{\sigma}_{L}: \mathcal{F}_{p_{1}, p_{2}}^{(L)} \mapsto \mathcal{F}_{-p_{1}, p_{2}}^{(L)}$ are defined by the conditions $\hat{\sigma}_{L} a_{m}=-a_{m} \hat{\sigma}_{L}, \hat{\sigma}_{L} b_{m}=+b_{m} \hat{\sigma}_{L}, \hat{\sigma}_{L}\left|p_{1}, p_{2}\right\rangle=$ $\left|-p_{1}, p_{2}\right\rangle$, and similar for $\hat{\sigma}_{R}$ (see, e.g., [44]).
} 
The twist parameter $k$ has a natural interpretation in the dual description - it can be identified with the so-called quasimomentum. The sine-Liouville Lagrangian is invariant under the transformation $\vartheta \mapsto \vartheta+\frac{2 \pi}{\sqrt{n+2}}$. Due to this periodicity, the space of states of the theory with the boundary conditions (2.69), splits on the orthogonal subspaces $\mathcal{H}_{k, m}$ such that for any state $|A\rangle \in \mathcal{H}_{k, m}$, the corresponding wave functional $\Psi_{A}[\varphi(x), \vartheta(x)]$ transforms as

$$
\Psi_{A}\left[\varphi(x), \vartheta(x)+\frac{2 \pi}{\sqrt{n+2}}\right]=\mathrm{e}^{2 \pi \mathrm{i} k} \Psi_{A}[\varphi(x), \vartheta(x)] .
$$

Let $P_{s}\left(\partial_{+} \varphi, \partial_{+} \vartheta, \ldots\right)$ be a local field of spin $s$, and a polynomial of $\partial_{+} \varphi, \partial_{+} \vartheta$ and their higher derivatives. All such fields are periodic in $x$, so that one can introduce the integral,

$$
\mathfrak{i}_{s-1}=\left(\frac{R}{2 \pi}\right)^{s-1} \int_{0}^{R} \frac{\mathrm{d} x}{2 \pi} P_{s}\left(\partial_{+} \varphi, \partial_{+} \vartheta, \ldots\right) .
$$

It turns out that for any even $s=2 j$ there exists a local density (defined modulo the addition of a total derivative and an overall multiplicative constant) such that $\mathfrak{i}_{2 j-1}$ is an integral of motion and satisfies the commutativity conditions

$$
\left[\mathfrak{i}_{2 j-1}, \tau(\lambda)\right]=\left[\mathfrak{i}_{2 j-1}, \mathfrak{i}_{2 j^{\prime}-1}\right]=0 \text {. }
$$

These operators are referred to as the (chiral) local IM. They were studied in ref. [42], where the explicit form for the first local IM and their vacuum eigenvalues, $i_{2 j-1}\left(p_{1}, p_{2}\right)$ for $j=1,2,3$, can be found. Here we only note that for any $j=1,2, \ldots$

$$
P_{2 j}=\sum_{l+m=j} C_{l m}^{(j)}\left(\partial_{+} \varphi\right)^{2 l}\left(\partial_{+} \vartheta\right)^{2 m}+\ldots
$$

where the dots stand for monomials which include higher derivatives of $\partial_{+} \varphi$ and $\partial_{+} \vartheta$ and the numerical coefficients $C_{l m}^{(s)}$ can be written as

$$
C_{l m}^{(j)}=C_{2 j-1} \frac{(-2)^{j+1}(2 j-2) !}{(j+1) !} \frac{\left((n+2)\left(\frac{1}{2}-j\right)\right)_{l}\left((-n)\left(\frac{1}{2}-j\right)\right)_{m}}{l ! m !}(-n)^{l-1}(n+2)^{m-1} .
$$

Here $(a)_{m}=\prod_{i=0}^{m-1}(a+i)$ is the Pochhammer symbol. The overall normalization constant $C_{2 j-1}$ is usually set to

$$
C_{2 j-1}=\frac{2^{-3 j}(j+1) ! n(n+2)}{\left((n+2)\left(\frac{1}{2}-j\right)\right)_{j}\left((-n)\left(\frac{1}{2}-j\right)\right)_{j}} .
$$

\section{Chiral transfer-matrix for $\mathbb{Z}_{n}$ parafermions}

While quantizing the sausage model within the BLZ approach, we have run into the problem of finding the spectrum of the chiral transfer-matrices for the cigar NLSM. As it has been explained, we can consider $\tau(\lambda)$ as an operator acting in the Fock space $\mathcal{F}_{\mathbf{p}}$ with real $\mathbf{p}=\left(p_{1}, p_{2}\right)$. From the formal point of view, the same spectral problem can be posed for any complex values of $\mathbf{p}$. Notice, that for real $p_{2}, \lambda^{2}$ and pure imaginary $p_{1}$, the operator 
$\tau(\lambda)$ is Hermitian. The spectral problem in this case (except for $p_{1}=0$ ) is not directly related to the quantization of the sausage model, however for $n=2,3, \ldots$ and a certain discrete set of $p_{1}$ and $p_{2}$, it gives a better understanding of the interplay between the BLZ approach and that based on the discretization of the quantum system. It will be the subject of our study here.

\subsection{Bosonization of $\mathbb{Z}_{n}$ parafermions}

Let us take a closer look at the vertex operators $V^{( \pm)}(2.21)$, which appear in the construction of the chiral transfer-matrices $\tau_{j}$. As it was already mentioned, the constants $c^{ \pm}$can be arbitrarily chosen. Let us set $c^{ \pm}=1$ and assume that $n \geq 2$ is a positive integer. Then, eq. (2.21) can be recognized as the bosonization relations for the Fateev-Zamolodchikov $\mathbb{Z}_{n}$ parafermions [48]. More precisely, as follows from the normalization condition (2.28), the chiral nonlocal fields

$$
\psi^{ \pm}=\sqrt{n} q^{-\frac{1}{2}} V^{ \pm}
$$

can be understood as canonically normalized parafermion currents,

$$
\left.\psi^{ \pm}\left(x_{2}\right) \psi^{\mp}\left(x_{1}\right)\right|_{x_{2} \rightarrow x_{1}+0} \sim 1 \times\left(x_{2}-x_{1}\right)^{-2 \Delta_{\psi}}
$$

of the conformal dimension $\Delta_{\psi}=1-\frac{1}{n}$.

The chiral algebra of parafermion currents was introduced by Fateev and Zamolodchikov in ref. [49], in the construction of the $\mathbb{Z}_{n}$ CFT models with central charge

$$
c_{n}=\frac{2(n-1)}{n+2}
$$

describing the multicritical points of the $\mathbb{Z}_{n}$ statistical systems (certain generalizations of the $\mathbb{Z}_{2}$ invariant Ising model) [50]. The chiral component of the Hilbert space of the $\mathbb{Z}_{n}$ CFT can be decomposed into irreps $\mathcal{V}_{j}$ of the chiral algebra. Here, the subscript $j$ stands for the highest weight of the irrep with highest weight vector $\left|\sigma_{\mathfrak{j}}\right\rangle$ having conformal dimension

$$
\Delta_{\mathfrak{j}}=\frac{\mathfrak{j}(n-2 \mathfrak{j})}{n(n+2)} .
$$

The admissible values of $\mathfrak{j}$ are given by non-negative integers and half-integers restricted by the condition

$$
\mathfrak{j}=0, \frac{1}{2}, 1, \frac{3}{2}, \ldots, \frac{1}{2}\left[\frac{n}{2}\right],
$$

where $\left[\frac{n}{2}\right]$ is the integer part of $n / 2$. The fundamental parafermion currents $\psi^{+}$and $\psi^{-}$ act in $\mathcal{V}_{j}$ and carry the $\mathbb{Z}_{n}$-charges +2 and -2 respectively:

$$
\Omega \psi^{ \pm} \Omega^{-1}=\omega^{ \pm 2} \psi^{ \pm}, \quad \text { where } \quad \omega=\mathrm{e}^{-\frac{2 \pi \mathrm{i}}{n}} .
$$

Note that $2 \mathbf{j}$ can be identified with the $\mathbb{Z}_{n}$-charge of the highest weight vector: ${ }^{6}$

$$
\Omega\left|\sigma_{\mathfrak{j}}\right\rangle=\omega^{2 \mathrm{j}}\left|\sigma_{\mathfrak{j}}\right\rangle .
$$

\footnotetext{
${ }^{6}$ To be more precise, the chiral component of the Hilbert space of the $\mathbb{Z}_{n}$ CFT contains, together with the irrep $\mathcal{V}_{j}^{(+)} \equiv \mathcal{V}_{\mathbf{j}}$, the irrep $\mathcal{V}_{j}^{(-)}$whose highest weight vector has the same conformal dimension (3.4) but carries the $\mathbb{Z}_{n}$-charge $\omega^{-2 \mathrm{j}}$. For even $n, \mathcal{V}_{\frac{n}{4}}^{(-)}=\mathcal{V}_{\frac{n}{4}}^{(+)}$. The chiral transfer-matrix (3.13) is a $\mathbb{Z}_{2}$-invariant operator which does not distinguish between the irreps $\mathcal{V}_{j}^{(+)}$and $\mathcal{V}_{j}^{(-)}$.
} 
The irrep $\mathcal{V}_{j}$ naturally splits on the subspaces $\mathcal{V}_{j}^{(\mathfrak{m})}$ characterized by a definite value of the $\mathbb{Z}_{n}$-charge,

$$
\mathcal{V}_{\mathbf{j}}=\left[\begin{array}{ll}
\oplus_{s=0}^{2 j} & \mathcal{V}_{j}^{(2 j-2 s)}
\end{array}\right] \oplus\left[\oplus_{s=1}^{n-2 j-1} \mathcal{V}_{j}^{(2 \mathfrak{j}+2 s)}\right]: \quad \Omega \mathcal{V}_{j}^{(\mathfrak{m})}=\omega^{\mathfrak{m}} \mathcal{V}_{\mathfrak{j}}^{(\mathfrak{m})}
$$

The lowest possible conformal dimension in the subspace $\mathcal{V}_{j}^{(\mathfrak{m})}$ is given by $\Delta_{\mathfrak{j}, \mathfrak{m}}$ for $\mathfrak{m}=$ $-2 \mathfrak{j},-2 \mathfrak{j}+2, \ldots, 2 \mathfrak{j}$, and $\Delta_{\mathfrak{j}, \mathfrak{m}}+\frac{1}{2}(\mathfrak{m}-2 \mathfrak{j})$ for $\mathfrak{m}=2 \mathfrak{j}+2, \ldots, 2 n-2 \mathfrak{j}-2$. Here we use the notation

$$
\Delta_{\mathfrak{j}, \mathfrak{m}}=\frac{\mathfrak{j}(\mathfrak{j}+1)}{n+2}-\frac{\mathfrak{m}^{2}}{4 n} .
$$

In what follows $\left|\sigma_{\mathfrak{j}, \mathfrak{m}}\right\rangle$ will denote the state from the subspace $\mathcal{V}_{\mathfrak{j}}^{(\mathfrak{m})}$ with $\mathfrak{m}=2 \mathfrak{j}, 2 \mathfrak{j}-$ $2, \ldots,-2 \mathfrak{j}$ of the lowest conformal dimension $\Delta_{\mathfrak{j}, \mathfrak{m}}$.

From the mathematical point of view the bosonization of the algebra of parafermion currents implies that the subspaces $\mathcal{V}_{\mathfrak{j}}^{(\mathfrak{m})}$ with $\mathfrak{m}=2 \mathfrak{j}, 2 \mathfrak{j}-2, \ldots,-2 \mathfrak{j}$ can be understood as a cohomology of the Fock space $\mathcal{F}_{p_{1}, p_{2}}$ where

$$
p_{1}=\frac{\mathrm{i}}{2} \mathfrak{m}, \quad p_{2}=\mathfrak{j}+\frac{1}{2},
$$

with respect to a certain BRST complex à la the Felder complex [51] involved in the bosonization of the highly reducible Verma modules over the Virasoro algebra. Among other things, the bosonization formula (3.1) leads to the following relation for the matrix elements of the parafermion currents:

$$
\left\langle\sigma_{\mathfrak{j}, \mathfrak{m}}\left|\prod_{m=1}^{M} \psi^{\varepsilon_{m}}\left(x_{m}\right)\right| \sigma_{\mathfrak{j}, \mathfrak{m}}\right\rangle=\left(n q^{-1}\right)^{\frac{M}{2}}\left\langle p_{1}, p_{2}\left|\prod_{m=1}^{M} V^{\varepsilon_{m}}\left(x_{m}\right)\right| p_{1}, p_{2}\right\rangle \quad\left(\varepsilon_{m}= \pm\right)
$$

provided $\sum_{m=1}^{L} \varepsilon_{m}=0$ and the pairs $(\mathfrak{j}, \mathfrak{m})$ are related to $\left(p_{1}, p_{2}\right)$ as in eq. (3.10). It is not difficult to see that the $\mathbb{Z}_{n}$-charge operator is bosonized by the relation

$$
\Omega=\mathrm{e}^{4 \pi P_{1}}
$$

and the operator $\tau(\lambda)$ can be written in the form

$$
\tau(\lambda)=\operatorname{Tr}\left[\overleftarrow{\mathcal{P}} \exp \left(\mathrm{i} \frac{\lambda_{+}}{\sqrt{n}} \int_{t_{0}}^{t_{0}+R} \mathrm{~d} x\left(\psi^{+} \sigma_{+}+\psi^{-} \sigma_{-}\right)\right) \Omega^{-\frac{1}{2} \sigma_{3}}\right]
$$

Therefore, $\tau(\lambda)$ can be understood as an operator which invariantly acts in the subspaces $\mathcal{V}_{j}^{(\mathfrak{m})}$ of the irrep $\mathcal{V}_{j}$ of the algebra of parafermion currents. We can now address the problem of the diagonalization of this operator. Notice that it is sufficient to consider $\mathfrak{m} \geq 0$, and in what follows we will always assume that

$$
\begin{aligned}
\mathfrak{j} & =0, \frac{1}{2}, 1, \frac{3}{2}, \ldots, \frac{1}{2}\left[\frac{n}{2}\right] \\
\mathfrak{m} & =2 \mathfrak{j}, 2 \mathfrak{j}-2, \ldots, 2 \mathfrak{j}-2[\mathfrak{j}] .
\end{aligned}
$$




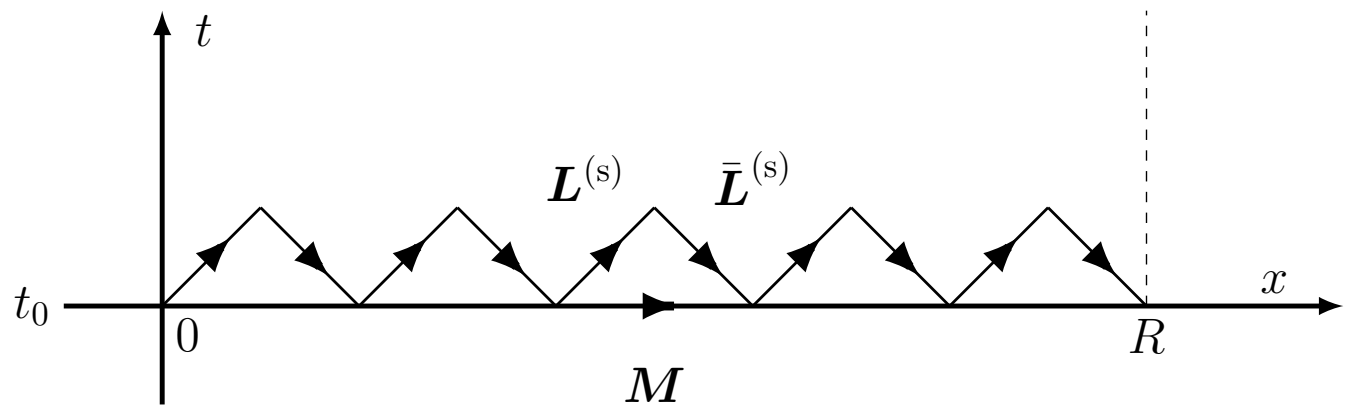

Figure 5. By replacing the integration over the segment at the time slice $t=t_{0}$ by integration over the light-cone pieces, the monodromy matrix can be expressed as a product of the elementary "light-cone" transport matrices: $\boldsymbol{M}(\mu)=\overleftarrow{\coprod}_{s=1}^{N} \overline{\boldsymbol{L}}^{(\mathrm{s})}(\mu) \boldsymbol{L}^{(\mathrm{s})}(\mu)$

\subsection{Discretization of the chiral transfer-matrix}

The goal of this section is to propose a lattice version of the parafermionic chiral transfermatrix (3.13). For this purpose we return back to the formula (1.20) for the classical conserved charges in the sausage model and follow the approach based on discretization that was mentioned in the introduction.

Split the integration contour onto $N$ small segments of size $\delta$ and consider the elementary transport matrices in the fundamental representation:

$$
\boldsymbol{M}^{(\mathrm{s})}(\mu)=\pi_{\frac{1}{2}}\left[\overleftarrow{\mathcal{P}} \exp \int_{x_{\mathrm{s}}-\delta / 2}^{x_{\mathrm{s}}+\delta / 2} \mathrm{~d} x \boldsymbol{A}_{x}\right] \quad(\mathrm{s}=1, \ldots, N)
$$

These can be expressed in terms of the elementary "light-cone" transport matrices $\boldsymbol{M}^{(\mathrm{s})}(\mu)=\overline{\boldsymbol{L}}^{(\mathrm{s})}(\mu) \boldsymbol{L}^{(\mathrm{s})}(\mu)$ (see figure 5) and, as it follows from eq. (1.7),

$$
\begin{aligned}
& \left\{\boldsymbol{L}^{(\mathrm{s})}(\mu) \otimes \boldsymbol{L}^{\left(\mathrm{s}^{\prime}\right)}\left(\mu^{\prime}\right)\right\}=\left[\boldsymbol{L}^{(\mathrm{s})}(\mu) \otimes \boldsymbol{L}^{\left(\mathrm{s}^{\prime}\right)}\left(\mu^{\prime}\right), \boldsymbol{r}_{\frac{1}{2} \frac{1}{2}}\left(\mu / \mu^{\prime}\right)\right] \delta_{\mathrm{ss}^{\prime}} \\
& \left\{\overline{\boldsymbol{L}}^{(\mathrm{s})}(\mu) \otimes \overline{\boldsymbol{L}}^{\left(\mathrm{s}^{\prime}\right)}\left(\mu^{\prime}\right)\right\}=\left[\overline{\boldsymbol{L}}^{(\mathrm{s})}(\mu) \otimes \overline{\boldsymbol{L}}^{\left(\mathrm{s}^{\prime}\right)}\left(\mu^{\prime}\right), \boldsymbol{r}_{\frac{1}{2} \frac{1}{2}}\left(\mu / \mu^{\prime}\right)\right] \delta_{\mathrm{ss}^{\prime}} \\
& \left\{\boldsymbol{L}^{(\mathrm{s})}(\mu) \otimes \overline{\boldsymbol{L}}^{\left(\mathrm{s}^{\prime}\right)}\left(\mu^{\prime}\right)\right\}=0,
\end{aligned}
$$

where

$$
\boldsymbol{r}_{\frac{1}{2} \frac{1}{2}}(\mu)=\left(\begin{array}{cccc}
a(\mu) & 0 & 0 & 0 \\
0 & 0 & c(\mu) & 0 \\
0 & c(\mu) & 0 & 0 \\
0 & 0 & 0 & a(\mu)
\end{array}\right) \quad \text { with } \quad \begin{aligned}
& a(\mu)=\frac{1}{2} \frac{\mu+\mu^{-1}}{\mu-\mu^{-1}} \\
& c(\mu)=\frac{1}{\mu-\mu^{-1}}
\end{aligned}
$$

Consider the structure of $\boldsymbol{L}^{(\mathrm{s})}(\mu)=\pi_{\frac{1}{2}}\left[\overleftarrow{\mathcal{P}} \exp \int_{x_{\mathrm{s}}-\delta / 2}^{x_{\mathrm{s}}+\delta / 2} \mathrm{~d} x \boldsymbol{A}_{+}\right]$. From the explicit form of $\boldsymbol{A}_{+}$(1.14), one has

$$
\boldsymbol{L}(\mu)=\left(\begin{array}{cc}
1+\frac{f(\mu)}{4} H & \frac{\mathrm{i}}{2} g(\mu) E_{-} \\
\frac{\mathrm{i}}{2} g(\mu) E_{+} & 1-\frac{f(\mu)}{4} H
\end{array}\right)+O\left(\delta^{2}\right),
$$


with

$$
E_{ \pm}=\int_{x_{\mathrm{s}}-\delta / 2}^{x_{\mathrm{s}}+\delta / 2} \mathrm{~d} x \Pi_{+}(x) \mathrm{e}^{\mp Q(x)}, \quad H=2 \int_{x_{\mathrm{s}}-\delta / 2}^{x_{\mathrm{s}}+\delta / 2} \mathrm{~d} x \Pi_{+}(x),
$$

and, as it follows from the canonical commutation relations,

$$
\left\{E_{+}, E_{-}\right\}=-H, \quad\left\{H, E_{ \pm}\right\}= \pm 2 E_{ \pm}
$$

Here, to simplify the notation, we have temporarily dropped the superscript "s" and are focusing on a single site. Let's look at the above formulae from a slightly different angle. Suppose we are given the matrices $\boldsymbol{L}$ of the form (3.18) with arbitrary functions $g(\mu)$ and $f(\mu)$ where $H, E_{ \pm}$satisfy the Poisson bracket relations (3.20). The requirement that $\boldsymbol{L}$ obeys the Yang-Baxter Poisson algebra (3.16) leads to two equations imposed on the functions $f$ and $g$ :

$$
\begin{aligned}
a(\mu / \lambda) g(\lambda)-c(\mu / \lambda) g(\mu) & =\frac{1}{2} g(\lambda) f(\mu) \\
c(\mu / \lambda) & =\frac{1}{2} \frac{g(\lambda) g(\mu)}{f(\lambda)-f(\mu)} .
\end{aligned}
$$

One can show that, modulo the rescaling $\mu \mapsto$ const $\mu$, the most general solution to these equations is given by formula (1.13) with $\kappa$ an arbitrary parameter.

This simple calculation hints as to how we should proceed with the deduction of the quantum counterpart of the above formulae. Strictly speaking, there is no canonical prescription for the quantization of the Poisson brackets (3.20), however, it seems natural to substitute them by the defining relations of the $U_{q}(\mathfrak{s l}(2))$ quantum algebra with $q=\mathrm{e}^{\mathrm{i} \hbar}$ :

$$
\left[\mathrm{E}_{+}, \mathrm{E}_{-}\right]=\frac{q^{\mathrm{H}}-q^{-\mathrm{H}}}{q-q^{-1}}, \quad\left[\mathrm{H}, \mathrm{E}_{ \pm}\right]= \pm 2 \mathrm{E}_{ \pm}
$$

For the quantum version of eq. (3.18), we put forward the following ansatz

$$
\boldsymbol{L}(\mu)=\left(\begin{array}{cc}
F_{-}(\mu) q^{-\frac{1}{2} \mathrm{H}}+F_{+}(\mu) q^{+\frac{1}{2} \mathrm{H}} & \left(q-q^{-1}\right) G(\mu) \mathrm{E}_{-} \\
\left(q-q^{-1}\right) G(\mu) \mathrm{E}_{+} & F_{-}(\mu) q^{+\frac{1}{2} \mathrm{H}}+F_{+}(\mu) q^{-\frac{1}{2} \mathrm{H}}
\end{array}\right)
$$

where $F_{ \pm}(\mu)$ and $G(\mu)$ are some functions. The classical matrix will be recovered if we assume that as $\hbar \rightarrow 0$,

$$
q^{ \pm \frac{1}{2} \mathrm{H}}=1 \pm \frac{1}{4} \mathrm{i} \hbar \mathrm{H}+o(\delta)
$$

while

$$
\mathrm{H}=-\mathrm{i} \hbar^{-1} H+O\left(\hbar^{0}\right), \quad \mathrm{E}^{( \pm)}=\hbar^{-1} E^{( \pm)}+O\left(\hbar^{0}\right),
$$

and also

$$
\lim _{\hbar \rightarrow 0} F_{ \pm}(\mu)=\frac{1}{2}(1 \pm f(\mu)), \quad \lim _{\hbar \rightarrow 0} G(\mu)=\frac{1}{2} g(\mu) .
$$

It is clear that the operator valued matrix $\boldsymbol{L}$ must satisfy the Yang-Baxter algebra

$$
\boldsymbol{R}_{\frac{1}{2} \frac{1}{2}}\left(\mu^{\prime} / \mu\right)(\boldsymbol{L}(\mu) \otimes 1)\left(1 \otimes \boldsymbol{L}\left(\mu^{\prime}\right)\right)=\left(1 \otimes \boldsymbol{L}\left(\mu^{\prime}\right)\right)(\boldsymbol{L}(\mu) \otimes 1) \boldsymbol{R}_{\frac{1}{2} \frac{1}{2}}\left(\mu^{\prime} / \mu\right),
$$


where $\boldsymbol{R}_{\frac{1}{2} \frac{1}{2}}$ is given by eq. (2.32). The ansatz (3.23), combined with this relation, yields

$$
F_{+}(\mu)=+a \mu G(\mu), \quad F_{-}(\mu)=-(a \mu)^{-1} G(\mu),
$$

where $a$ is an arbitrary constant. Consistency with eq. (3.26) and the explicit form for $f$ and $g(1.13)$ requires that

$$
\lim _{\hbar \rightarrow 0}\left(a^{2}\right)=\frac{1-\kappa}{1+\kappa} .
$$

We may now use the well known fact that the $U_{q}(\mathfrak{s l}(2))$ algebra with defining relations (3.22) and $q=\mathrm{e}^{\mathrm{i} \hbar} \frac{\mathrm{i}}{2}$ admits a formal realization in terms of the Heisenberg algebra

$$
[\mathrm{Q}, \mathrm{P}]=\mathrm{i} \hbar
$$

Namely [52],

$$
\begin{aligned}
& \mathrm{E}_{+}=\mathrm{e}^{-\frac{1}{2} \mathrm{Q}} \frac{\sinh \left(\frac{1}{2} \mathrm{P}+\hbar C\right)}{\sin \left(\frac{1}{2} \hbar\right)} \mathrm{e}^{-\frac{1}{2} \mathrm{Q}} \\
& \mathrm{E}_{-}=\mathrm{e}^{+\frac{1}{2} \mathrm{Q}} \frac{\sinh \left(\frac{1}{2} \mathrm{P}-\hbar C\right)}{\sin \left(\frac{1}{2} \hbar\right)} \mathrm{e}^{+\frac{1}{2} \mathrm{Q}} \\
& \mathrm{H}=-2 \mathrm{i} \hbar^{-1} \mathrm{P} .
\end{aligned}
$$

It is not difficult to see that this can be thought of as the quantum counterpart of eqs. (3.19). The constant $C$ is arbitrary and is related to the value of the quantum Casimir. In fact, it is convenient to substitute it by $\ell: C=\mathrm{i}(2 \ell+1) / 4$, then

$$
\frac{1}{2}\left[\left(q+q^{-1}\right)\left(q^{\mathrm{H}}+q^{-\mathrm{H}}\right)+\left(q-q^{-1}\right)^{2}\left(\mathrm{E}_{-} \mathrm{E}_{+}+\mathrm{E}_{+} \mathrm{E}_{-}\right)\right]=q^{2 \ell+1}+q^{-2 \ell-1} .
$$

Let us introduce the Heisenberg group generators, subject to the Weyl commutation relations

$$
\mathrm{V}=\exp \left(\frac{1}{2} \mathrm{P}\right), \quad \mathrm{U}=\exp (\mathrm{Q}): \quad \mathrm{UV}=q \mathrm{VU}
$$

Our analysis suggests that the $2 \times 2$ operator valued matrix $\boldsymbol{L}(\mu)=\mathcal{L}^{(\ell)}(\mu \mid \mathrm{U}, \mathrm{V})$, where [53, $54]$

$$
\mathcal{L}^{(\ell)}(\mu \mid \mathrm{U}, \mathrm{V})=\left(\begin{array}{cc}
\left(\mu \mathrm{V}-\mu^{-1} \mathrm{~V}^{-1}\right) & \mathrm{i}\left(q^{-\ell} \mathrm{V}-q^{+\ell} \mathrm{V}^{-1}\right) \mathrm{U} \\
\mathrm{i}\left(q^{+\ell} \mathrm{V}-q^{-\ell} \mathrm{V}^{-1}\right) \mathrm{U}^{-1} & \left(\mu \mathrm{V}^{-1}-\mu^{-1} \mathrm{~V}\right)
\end{array}\right)
$$

satisfies the Yang-Baxter relation (3.27). Furthermore, it is easy to see that the same properties still hold for the matrix which depends on a set of six parameters $\{a, b, c, g, r, \ell\}$ :

$$
\mathcal{L}\left[\begin{array}{l}
g r \ell \\
a b c
\end{array}\right](\mu \mid \mathrm{U}, \mathrm{V})=g r^{\frac{\sigma_{3}}{2}} \mathcal{L}^{(\ell)}(a \mu \mid 6 \mathrm{U}, c \mathrm{~V}) r^{\frac{\sigma_{3}}{2}}
$$

Most of the parameters, except maybe $r$ and $\ell$, look trivial when we consider only a single site. However, for the discretized system of $N$ sites, the possibility that the parameters may be different at different sites should be considered. In any case, one can expect that 
for a properly adjusted set $\left\{a_{\mathrm{s}}, b_{\mathrm{s}}, c_{\mathrm{s}}, g_{\mathrm{s}}, r_{\mathrm{s}}, \ell_{\mathrm{s}}\right\}_{\mathrm{s}=1}^{N}$ the discretized quantum version of the formulae $(2.9),(2.10)$ in the fundamental representation is given by

$$
\mathcal{T}^{(N)}(\mu)=(-\mu)^{N} \operatorname{Tr}\left[\overleftarrow{\mathcal{P}}\left(\prod_{\mathrm{s}=1}^{N} \mathcal{L}\left[\begin{array}{l}
g_{\mathrm{s}} r_{\mathrm{s}} \ell_{\mathrm{s}} \\
a_{\mathrm{s}} b_{\mathrm{s}} c_{\mathrm{s}}
\end{array}\right]\left(\mu \mid \mathrm{U}_{\mathrm{s}}, \mathrm{V}_{\mathrm{S}}\right)\right)\left(q^{d} \mathrm{~V}\right)^{-\sigma_{3}}\right]
$$

where $\mathrm{V}=\prod_{\mathrm{s}=1}^{N} \mathrm{~V}_{\mathrm{s}}$ is the discretized counterpart to the exponential $\mathrm{e}^{\pi P_{1}}$ and $d$ is some constant. The overall factor $(-\mu)^{N}$ is inserted to ensure that the transfer-matrix is a polynomial in $\mu^{2}$ of order $N$. Notice that

$$
\mathcal{T}^{(N)}(0)=\mathrm{V}^{-2} q^{-d} \prod_{s=1}^{N} g_{\mathrm{s}} r_{\mathrm{s}} c_{\mathrm{s}}^{-1}+\mathrm{V}^{+2} q^{d} \prod_{s=1}^{N} g_{\mathrm{s}} r_{\mathrm{s}}^{-1} c_{\mathrm{s}}
$$

is expressed in terms of integer powers of

$$
\mathrm{X}_{\mathrm{s}} \equiv \mathrm{V}_{\mathrm{S}}^{2}
$$

rather than $\mathrm{V}_{\mathrm{s}}$. In fact, this is true for any $\mu$, and it can be made explicit by rewriting (3.36) in the equivalent form:

$$
\mathcal{T}^{(N)}(\mu)=\mathscr{C} \operatorname{Tr}\left[\overleftarrow{\mathcal{P}}\left(\prod_{j=1}^{N}\left(\mathcal{L}_{-}^{\left(r_{\mathrm{s}} \ell_{\mathrm{s}}\right)}\left(b_{\mathrm{S}} \mathrm{U}_{\mathrm{s}}, c_{\mathrm{S}}^{2} \mathrm{X}_{\mathrm{s}}\right)-a_{\mathrm{s}}^{2} \mu^{2} \mathcal{L}_{+}^{\left(r_{\mathrm{s}} \ell_{\mathrm{s}}\right)}\left(b_{\mathrm{s}} \mathrm{U}_{\mathrm{s}}, c_{\mathrm{s}}^{2} \mathrm{X}_{\mathrm{s}}\right)\right)\right)\left(\begin{array}{cc}
q^{-d} \mathrm{Z}^{-1} & 0 \\
0 & q^{d}
\end{array}\right)\right]
$$

where $\mathscr{C}$ is a constant, $\mathcal{L}_{ \pm}$are triangular matrices:

$$
\begin{aligned}
& \mathcal{L}_{-}^{(r \ell)}(\mathrm{U}, \mathrm{X})=\left(\begin{array}{cc}
r & 0 \\
-\mathrm{i}\left(q^{-\ell-1}-q^{+\ell-1} \mathrm{X}\right) \mathrm{U}^{-1} & r^{-1} \mathrm{X}
\end{array}\right) \\
& \mathcal{L}_{+}^{(r \ell)}(\mathrm{U}, \mathrm{X})=\left(\begin{array}{cc}
r \mathrm{X} \mathrm{\textrm {i }}\left(q^{1+\ell}-q^{1-\ell} \mathrm{X}\right) \mathrm{U} \\
0 & r^{-1}
\end{array}\right),
\end{aligned}
$$

and

$$
\mathrm{Z}=\prod_{\mathrm{s}=1}^{N} \mathrm{x}_{\mathrm{s}}
$$

Finally, let us note that the set of formal operators $\left\{\mathrm{U}_{\mathrm{s}}, \mathrm{X}_{\mathrm{S}}\right\}_{\mathrm{s}=1}^{N}$ satisfy the commutation relations

$$
\left[\mathrm{U}_{\mathrm{s}}, \mathrm{U}_{\mathrm{s}^{\prime}}\right]=\left[\mathrm{X}_{\mathrm{s}}, \mathrm{X}_{\mathrm{s}^{\prime}}\right]=\left[\mathrm{U}_{\mathrm{s}}, \mathrm{X}_{\mathrm{s}^{\prime}}\right]=0 \quad\left(\mathrm{~s} \neq \mathrm{s}^{\prime}\right), \quad \mathrm{U}_{\mathrm{s}} \mathrm{X}_{\mathrm{s}}=q^{2} \mathrm{X}_{\mathrm{s}} \mathrm{U}_{\mathrm{s}}
$$

and also that $\mathrm{Z}$ commutes with $\mathcal{T}^{(N)}(\mu)$ for any values of the parameters in (3.39).

We are now faced with the task of specifying the parameters in (3.39). Our analysis outlined below shows that in all likelihood it is enough to consider the case with

$$
\mathscr{C}=a_{\mathrm{S}}=b_{\mathrm{s}}=c_{\mathrm{s}}=r_{\mathrm{s}}=1, \quad \ell=-\frac{1}{2}, \quad d=2 p_{2}-1
$$


In this case, we expect that, with a properly chosen representation of the algebra (3.42) and with a properly understood scaling limit, the operator $\mathcal{T}^{(N)}(\mu)$ can be identified with the chiral transfer-matrix $\tau(\lambda)$ defined in eq. (2.33) with $j=\frac{1}{2}$. The discretized operator should be restricted to the sector with

$$
\mathrm{Z}=q^{1-2\left(p_{2}+\mathrm{i} p_{1}\right)},
$$

where, perhaps, some constraints need to be imposed on $\left(p_{1}, p_{2}\right)$. Recall that the pair $\left(p_{1}, p_{2}\right)$ label the Fock space $\mathcal{F}_{p_{1}, p_{2}}$ in which $\tau(\lambda)$ acts.

We came to the above conjecture through the analysis of the case of integer $n \equiv \frac{2 \pi}{\hbar}$. At $n=2,3, \ldots$, the formal algebra (3.42) admits an $n^{N}$ dimensional representation where the operators associated with each site are given by the $n \times n$ matrices

$$
\mathrm{X}_{\beta}^{\alpha}=\delta_{\alpha+1, \beta}, \quad \mathrm{U}_{\beta}^{\alpha}=\omega^{\alpha} \delta_{\alpha, \beta}, \quad \omega=\mathrm{e}^{-\frac{2 \pi \mathrm{i}}{n}} .
$$

Here $\alpha, \beta=0, \ldots, n-1$ and

$$
\delta_{\alpha \beta}=\left\{\begin{array}{lll}
1, & \alpha=\beta & (\bmod n) \\
0, & \alpha \neq \beta & (\bmod n)
\end{array} .\right.
$$

Now $\mathcal{T}^{(N)}(\mu)$ is an $n^{N} \times n^{N}$ matrix. We plan to discuss the diagonalization of the operator (3.39), with the parameters $\left\{a_{\mathrm{s}}, b_{\mathrm{s}}, c_{\mathrm{s}}, r_{\mathrm{s}}, \ell_{\mathrm{s}}\right\}$ not depending on "s", in a separate publication. Here we only note that such an operator but without the diagonal matrix $\left(\begin{array}{cc}q^{-d} \mathrm{Z}^{-1} & 0 \\ 0 & q^{d}\end{array}\right)$, was studied in the works $[54,55]$ in the context of the chiral Potts model. The extra diagonal matrix does not significantly change the diagonalization procedure.

In the case 3.43 , the diagonalization problem simplifies dramatically and can be solved within the standard Bethe ansatz framework (see next section for some details). This allows a thorough investigation of the scaling limit. An important point is that the scaling procedure requires a choice of some reference state ("vacuum") and only states whose "energy" measured from the vacuum energy remains finite as $N \rightarrow \infty$, should be taken into account. Let $\mathcal{H}_{M}^{(N)}$ be the subspace in the tensor product $\mathcal{H}^{(N)} \equiv\left(\mathbb{C}^{n}\right)^{\otimes N}$ with $\mathrm{Z}=\omega^{M}$. The operator $\mathcal{T}^{(N)}(\mu)$, with parameters as in eq. (3.43), restricted to the $\mathcal{H}_{\mathbf{j}-\frac{\mathrm{m}}{2}}^{(N)}$ subspace, with $\mathfrak{j}$ and $\mathfrak{m}$ satisfying the conditions (3.14), commutes with the Hamiltonian of the Fateev-Zamolodchikov $\mathbb{Z}_{n}$ spin chain

$$
\mathbb{H}^{(N)}=-\left.\frac{1}{n} \sum_{\mathrm{s}=1}^{N} \sum_{l=1}^{n-1} \frac{\left(\mathrm{X}_{\mathrm{s}}\right)^{l}+\left(\mathrm{U}_{\mathrm{s}} \mathrm{U}_{\mathrm{s}+1}^{\dagger}\right)^{l}}{\sin \left(\frac{\pi l}{n}\right)}\right|_{\mathrm{Z}=\omega^{\mathrm{j}-\frac{\mathrm{m}}{2}}}
$$

with twisted boundary conditions

$$
\mathrm{U}_{N+1}=\omega^{\mathfrak{j}+\frac{\mathfrak{m}}{2}} \mathrm{U}_{1}
$$

It also commutes with the lattice shift operator

$$
\mathbb{P}^{(N)}=\left.\delta_{\beta_{2}}^{\alpha_{1}} \delta_{\beta_{3}}^{\alpha_{2}} \ldots \delta_{\beta_{1}+\mathfrak{j}+\frac{\mathfrak{m}}{2}}^{\alpha_{N}}\right|_{\mathrm{z}=\omega^{\mathfrak{j}-\frac{\mathfrak{m}}{2}}} .
$$


Our numerical work for the vacuum state of the Hamiltonian $\mathbb{H}^{(N)}$ in the sector $\mathcal{H}_{j-\frac{\mathfrak{m}}{2}}^{(N)}$ for different admissible values of $n, \mathfrak{j}$ and $\mathfrak{m}$ gives strong support to the following relations (see appendix A)

$$
\tau(\lambda)=\operatorname{sim}_{N \rightarrow \infty} F^{(N)}(\lambda) \mathcal{T}^{(N)}\left(\left(\frac{\pi}{N}\right)^{\frac{1}{n}} \lambda\right),
$$

where

$$
F^{(N)}(\lambda)= \begin{cases}\exp \left(\sum_{l=1}^{\left[\frac{n}{2}\right]} \frac{\pi^{\frac{2 l}{n}}}{l \cos \left(\frac{\pi l}{n}\right)} N^{1-\frac{2 l}{n}} \lambda^{2 l}\right) & (n \neq 2,4, \ldots) \\ \left(\frac{N \mathrm{e}}{\pi}\right)^{\frac{4}{n} \lambda^{n}} \exp \left(\sum_{l=1}^{\left[\frac{n}{2}\right]-1} \frac{\pi^{\frac{2 l}{n}}}{l \cos \left(\frac{\pi l}{n}\right)} N^{1-\frac{2 l}{n}} \lambda^{2 l}\right) & (n=2,4, \ldots)\end{cases}
$$

and the symbol "slim" in (3.50) stands for the scaling limit which assumes that only the low-energy states are taken into account. The operator $\tau(\lambda)$ in (3.50) should be understood as the chiral parafermionic transfer-matrix (3.13) acting in the space $\mathcal{V}_{j}^{(\mathfrak{m})}$ discussed in the previous subsection.

Similarly to (3.50), one can consider the scaling limit

$$
\left.\operatorname{sim}_{N \rightarrow \infty} F^{(N)}\left(\lambda^{-1}\right)(-1)^{N} \mu^{-2 N} \mathcal{T}^{(N)}(\mu)\right|_{\mu=(\pi / N)^{\frac{1}{n}} \lambda^{-1}} .
$$

This can be identified with the anti-chiral parafermionic transfer-matrix $\bar{\tau}\left(\lambda^{-1}\right)$ acting in the space $\overline{\mathcal{V}}_{j}^{(2 j)}$. Therefore, in the scaling limit, (at least some of) the low energy states of $\mathcal{H}_{\mathfrak{j}-\frac{\mathfrak{m}}{2}}^{(N)}$ organize into the sector $\mathcal{V}_{\mathfrak{j}}^{(\mathfrak{m})} \otimes \overline{\mathcal{V}}_{\mathbf{j}}^{(2 \mathrm{j})}$ of the $\mathbb{Z}_{n}$ CFT Hilbert space. In this sector the low energy spectrum of the Hamiltonian (3.47) and the corresponding eigenvalues of the lattice shift operator have the form

$$
\begin{aligned}
& E^{(N)}=e_{0} N+\frac{2 \pi}{N}\left(\Delta_{\mathfrak{j}, \mathfrak{m}}+\Delta_{\mathfrak{j}, 2 \mathfrak{j}}-\frac{c_{n}}{12}+L+\bar{L}\right)+o\left(N^{-1}\right) \\
& P^{(N)}=\exp \left(\frac{2 \pi \mathrm{i}}{N}\left(\Delta_{\mathfrak{j}, \mathfrak{m}}-\Delta_{\mathfrak{j}, 2 \mathfrak{j}}+L-\bar{L}\right)\right),
\end{aligned}
$$

where $e_{0}$ is some constant and $L$ and $\bar{L}$ are integers. The central charge and conformal dimensions are given by eqs. (3.3) and (3.9), respectively.

Returning to the formal operator $\mathcal{T}^{(N)}(\mu)$ (3.39)-(3.44) for arbitrary values of $n$, we note that the case of real $\left(p_{1}, p_{2}\right)$ is of prime interest to the cigar NLSM. Perhaps the most promising approach to the construction of a suitable representation of the algebra (3.42) and the diagonalization of $\mathcal{T}^{(N)}(\mu)$ is based on the method of separation of variables [56].

\section{Spectrum of the chiral transfer-matrix}

In the previous sections the construction of the chiral transfer-matrices has been discussed. We are now ready to tackle the calculation of their spectrum. A powerful approach to this problem is the ODE/IQFT correspondence and here, we'll illustrate the method by calculating the vacuum eigenvalues of $\tau(\lambda)$. 


\subsection{Operators $\zeta_{ \pm}(\theta)$}

As it was already mentioned, a comprehensive discussion of the diagonalization procedure of the discretized chiral transfer-matrix $\mathcal{T}^{(N)}(\mu)(3.39)-(3.44)$ will be dealt with in a separate publication. However, it would be useful here to make a short summary of the important integrable structures which play a crucial rôle in the procedure. Namely, it is possible to explicitly construct two matrices $\mathcal{Z}_{ \pm}(\mu)$ satisfying the following set of conditions: ${ }^{7}$

(i) Commutativity

$$
\begin{array}{r}
{\left[\mathcal{Z}_{ \pm}(\mu), \mathcal{Z}_{ \pm}\left(\mu^{\prime}\right)\right]=\left[\mathcal{Z}_{+}(\mu), \mathcal{Z}_{-}\left(\mu^{\prime}\right)\right]=0} \\
{\left[\mathcal{Z}_{ \pm}(\mu), \mathcal{T}^{(N)}\left(\mu^{\prime}\right)\right]=\left[\mathcal{Z}_{ \pm}(\mu), \mathbb{H}^{(N)}\right]=\left[\mathcal{Z}_{ \pm}(\mu), \mathbb{P}^{(N)}\right]=0}
\end{array}
$$

(ii) "Quantum Wronskian" type relations

Odd $n$ :

$$
(1+\mu)^{2 N} \mathcal{Z}_{+}\left(q^{-\frac{1}{2}} \mu\right) \mathcal{Z}_{+}\left(q^{+\frac{1}{2}} \mu\right)-(1-\mu)^{2 N} \mathcal{Z}_{-}\left(q^{-\frac{1}{2}} \mu\right) \mathcal{Z}_{-}\left(q^{+\frac{1}{2}} \mu\right)=W(\mu) \mathbb{P}^{(N)}
$$

Even $n$ :

$$
\mathcal{Z}_{+}\left(q^{-\frac{1}{2}} \mu\right) \mathcal{Z}_{+}\left(q^{+\frac{1}{2}} \mu\right)-\left(1-\mu^{2}\right)^{2 N} \mathcal{Z}_{-}\left(q^{-\frac{1}{2}} \mu\right) \mathcal{Z}_{-}\left(q^{+\frac{1}{2}} \mu\right)=W(\mu) \mathbb{P}^{(N)}
$$

with

$$
W(\mu)=\left(1+\mu^{n}\right)^{2 N}-\left(1-\mu^{n}\right)^{2 N}
$$

(iii) " $T-Q$ " type relations

Odd $n$ :

$$
\mathcal{T}^{(N)}(\mu) \mathcal{Z}_{ \pm}(\mu)=\left(1 \mp q^{-\frac{1}{2}} \mu\right)^{2 N} \quad \mathcal{Z}_{\mp}\left(q^{-1} \mu\right)+\left(1 \mp q^{+\frac{1}{2}} \mu\right)^{2 N} \mathcal{Z}_{\mp}\left(q^{+1} \mu\right)
$$

Even $n$ :

$$
\begin{aligned}
& \mathcal{T}^{(N)}(\mu) \mathcal{Z}_{-}(\mu)=\mathcal{Z}_{+}\left(q^{-1} \mu\right)+\mathcal{Z}_{+}\left(q^{+1} \mu\right) \\
& \mathcal{T}^{(N)}(\mu) \mathcal{Z}_{+}(\mu)=\left(1-q^{-1} \mu^{2}\right)^{2 N} \mathcal{Z}_{-}\left(q^{-1} \mu\right)+\left(1-q^{+1} \mu^{2}\right)^{2 N} \mathcal{Z}_{-}\left(q^{+1} \mu\right)
\end{aligned}
$$

(iv) Analytical conditions

Odd $n$ :

$$
\mathcal{Z}_{ \pm}(\mu)=\mu^{\mathfrak{m}} \times(\text { polynomial in } \mu \text { of degree }(n-1) N-2 \mathfrak{j}-\mathfrak{m})
$$

Even $n$ :

$$
\begin{aligned}
& \mathcal{Z}_{+}(\mu)=\mu^{\mathfrak{m}} \times\left(\text { polynomial in } \mu^{2} \text { of degree } \frac{1}{2} n N-\mathfrak{j}-\frac{1}{2} \mathfrak{m}\right) \\
& \mathcal{Z}_{-}(\mu)=\mu^{\mathfrak{m}} \times\left(\text { polynomial in } \mu^{2} \text { of degree } \frac{1}{2}(n-2) N-\mathfrak{j}-\frac{1}{2} \mathfrak{m}\right)
\end{aligned}
$$

\footnotetext{
${ }^{7}$ All matrices appearing below are understood as operators acting invariantly in $\mathcal{H}_{\mathrm{j}-\frac{\mathfrak{m}}{2}}^{(N)}$ - the eigenspace
} of $\mathrm{Z}$ in $\left(\mathbb{C}^{n}\right)^{\otimes N}$ corresponding to the eigenvalue $\omega^{\mathbf{j}-\frac{\mathfrak{m}}{2}}$, where $\mathfrak{j}$ and $\mathfrak{m}$ are restricted as in (3.14). 
(v) $\boldsymbol{\mu} \rightarrow-\boldsymbol{\mu}$ symmetry

$$
\begin{array}{ll}
\text { Odd } n: & \mathcal{Z}_{ \pm}(-\mu)=(-1)^{\mathfrak{m}} \mathcal{Z}_{\mp}(\mu) \\
\text { Even } n: & \mathcal{Z}_{ \pm}(-\mu)=(-1)^{\mathfrak{m}} \mathcal{Z}_{ \pm}(\mu)
\end{array}
$$

The scaling limit of the operators $\mathcal{Z}_{ \pm}(\mu)$ is of special interest. In appendix B we present evidence that the following scaling limits, similar to (3.50), do exist:

$$
\begin{gathered}
\text { Odd } n: \zeta_{ \pm}=\lambda^{\mp 2 \lambda^{n}} \operatorname{sim}_{N \rightarrow \infty} G^{(N)}( \pm \lambda) \mathcal{Z}_{ \pm}\left(\left(\frac{\pi}{N}\right)^{\frac{1}{n}} \lambda\right) \\
\text { Even } n: \zeta_{+}=\quad \operatorname{sim}_{N \rightarrow \infty} G_{+}^{(N)}(\lambda) \mathcal{Z}_{+}\left(\left(\frac{\pi}{N}\right)^{\frac{1}{n}} \lambda\right) \\
\zeta_{-}=\lambda^{4 \lambda^{n}} \operatorname{sim}_{N \rightarrow \infty} G_{-}^{(N)}(\lambda) \mathcal{Z}_{-}\left(\left(\frac{\pi}{N}\right)^{\frac{1}{n}} \lambda\right)
\end{gathered}
$$

with

$$
\begin{aligned}
& G^{(N)}(\lambda)=\left(\frac{\mathrm{e} N}{\pi}\right)^{-\frac{2(n-1)}{n} \lambda^{n}} \exp \left(\sum_{l=1}^{n-1}(-1)^{l+1} \frac{\pi^{\frac{l}{n}}}{l \cos \left(\frac{\pi l}{2 n}\right)} N^{1-\frac{l}{n}} \lambda^{l}\right) \\
& G_{+}^{(N)}(\lambda)=\left(\frac{\mathrm{e} N}{\pi}\right)^{-2 \lambda^{n}} \\
& G_{-}^{(N)}(\lambda)=\left(\frac{\mathrm{e} N}{\pi}\right)^{\frac{2(n-2)}{n} \lambda^{n}} \exp \left(-\sum_{l=1}^{\frac{n}{2}-1} \frac{\pi^{\frac{2 l}{n}}}{l \cos \left(\frac{\pi l}{n}\right)} N^{1-\frac{2 l}{n}} \lambda^{2 l}\right) .
\end{aligned}
$$

Notice that for odd $n$, we include the "strange" extra factor $\lambda^{\mp 2 \lambda^{n}}$ in the formula for $\zeta_{ \pm}$. A similar factor $\lambda^{4 \lambda^{n}}$ appears for $\zeta_{-}$with even $n$. At first glance they look artificial and, furthermore, make $\zeta_{ \pm}$multivalued functions of $\lambda$. However, these "strange" factors allow one to write the scaling version of the quantum Wronskian type relations (ii) in a form which is applicable for both odd and even $n$ :

$$
\zeta_{+}\left(\theta+\frac{\mathrm{i} \pi}{2}\right) \zeta_{+}\left(\theta-\frac{\mathrm{i} \pi}{2}\right)-\zeta_{-}\left(\theta+\frac{\mathrm{i} \pi}{2}\right) \zeta_{-}\left(\theta-\frac{\mathrm{i} \pi}{2}\right)=2 \sinh \left(2 \pi \mathrm{e}^{\theta}\right)
$$

Here we have introduced $\theta$,

$$
\lambda=\mathrm{e}^{\frac{\theta}{n}}
$$

and later we'll argue that $\zeta_{ \pm}$are single valued functions of this variable. The $T-Q$ type relations (iii) in the scaling limit also have the same form for odd and even $n$,

$$
\tau(\lambda) \zeta_{ \pm}(\theta)=\zeta_{\mp}(\theta-\mathrm{i} \pi)+\zeta_{\mp}(\theta+\mathrm{i} \pi) .
$$

Since the operator $\tau(\lambda)$ acts in the parafermionic space $\mathcal{V}_{j}^{(\mathfrak{m})}$ it is natural to expect that the same holds true for $\zeta_{ \pm}(\theta)$. 
The relations (4.4) and (4.6) work for $n=2,3,4 \ldots$, but is it possible to extend them to non-integer $n$ ? We conjecture, that for any $n>0$ and general values of $\left(p_{1}, p_{2}\right)$, there exists a pair of operators which act invariantly in the Fock level subspaces

$$
\zeta_{ \pm}(\theta): \quad \mathcal{F}_{p_{1}, p_{2}}^{(L)} \mapsto \mathcal{F}_{p_{1}, p_{2}}^{(L)}
$$

such that

$$
\left[\zeta_{ \pm}(\theta), \zeta_{ \pm}\left(\theta^{\prime}\right)\right]=\left[\zeta_{+}(\theta), \zeta_{-}\left(\theta^{\prime}\right)\right]=\left[\zeta_{ \pm}(\theta), \tau\left(\lambda^{\prime}\right)\right]=0,
$$

satisfying the relations (4.4) and (4.6).

Unfortunately, at this moment we don't know how to explicitly construct the operators (4.7). Nevertheless, there are strong physical arguments that they do exist. In the works [42] and [43], $\zeta_{+}$and $\zeta_{-}$were introduced and studied for real $\left(p_{1}, p_{2}\right)$, as the boundary state operators in the paperclip model with topological angle equal to 0 and $\pi$, respectively. ${ }^{8}$ Among the results of those works is the large- $\theta$ behaviour. It was proposed that the operators $\zeta_{ \pm}$possess the following asymptotic at $\theta \rightarrow+\infty$ :

$$
\begin{aligned}
& \zeta_{+}(\theta) \asymp \exp \left(-\left(2 \theta+\pi \cot \left(\frac{\pi n}{2}\right)-C\right) \mathrm{e}^{\theta}-\sum_{j=1}^{\infty} \mathfrak{i}_{2 j-1} \mathrm{e}^{-\theta(2 j-1)}\right) \\
& \zeta_{-}(\theta) \asymp \tilde{\tau}\left(\mathrm{e}^{-\frac{\theta}{n+2}}\right) \quad \exp \left(\left(2 \theta-\pi \cot \left(\frac{\pi n}{2}\right)-C\right) \mathrm{e}^{\theta}+\sum_{j=1}^{\infty} \mathfrak{i}_{2 j-1} \mathrm{e}^{-\theta(2 j-1)}\right) .
\end{aligned}
$$

Here $\tilde{\tau}(\tilde{\lambda})$ is the formal series (2.44) generating the set of dual nonlocal IM while $\left\{\mathfrak{i}_{2 j-1}\right\}_{j=1}^{\infty}$ is the infinite set of local IM (2.72)-(2.76). The real constant $C$ is non-universal and can be chosen at will. In ref. [42], it was set to $\pi \cot \left(\frac{\pi n}{2}\right)$. For our purposes it is convenient to set it to zero,

$$
C=0 \text {. }
$$

With this choice it turns out that for odd $n$ and $\left(p_{1}, p_{2}\right)$ restricted by the conditions (3.10), (3.14), $\zeta_{ \pm}$are the same functions that appear in the scaling limit (4.1). However, one can see from eq. (4.9) that the operators $\zeta_{ \pm}$become singular for even $n$. They can be analytically regularized similar to as in eq. (2.50),

$$
\left.\zeta_{ \pm}^{(\mathrm{reg})}(\theta)\right|_{n=2 l}=\left.\lim _{\epsilon \rightarrow 0} \exp \left(\frac{2}{\epsilon} \mathrm{e}^{\theta}\right) \zeta_{ \pm}(\theta)\right|_{n=2 l+\epsilon} \quad(l=1,2,3 \ldots)
$$

When the regularized operators are restricted to the parafermionic space $\mathcal{V}_{j}^{(\mathfrak{m})}$, they are the same as the ones on the left hand side of eq. (4.2). ${ }^{9}$

It is expected that the operators $\zeta_{ \pm}$are entire functions of $\theta$ (in the sense that all their matrix elements and eigenvalues are entire functions of this variable) satisfying, for real $\left(p_{1}, p_{2}\right)$, the Hermiticity condition

$$
\left[\zeta_{ \pm}(\theta)\right]^{\dagger}=\zeta_{ \pm}\left(\theta^{*}\right)
$$

\footnotetext{
${ }^{8}$ In the notations of ref. [42]: $\mathbb{B}(\kappa)=2^{-\frac{1}{2}} \exp \left(2 \theta \mathrm{e}^{\theta}\right) \zeta_{+}(\theta)$, provided $\kappa=\mathrm{e}^{\theta}$.

${ }^{9}$ Here, we have abused notation because the $\zeta_{ \pm}$in $(4.2)$ denote the continuous operators obtained by means of the lattice regularization procedure.
} 
As it was pointed out, the asymptotic formulae (4.9) are written for large positive $\theta$, however in all likelihood, they hold true for complex values, at least in the strip $|\Im m(\theta)|<\pi$ with $\Re e(\theta) \rightarrow+\infty$.

Also, it deserves mentioning that the chiral transfer-matrices $\tau_{j}(\lambda)$ with $j=0, \frac{1}{2}, 1, \ldots$, can be expressed in terms of the operators $\zeta_{ \pm}(\theta)$. Namely, for $j=\frac{1}{2}, \frac{3}{2}, \ldots$

$$
\begin{aligned}
& \tau_{j}(\lambda)=\frac{(-1)^{j+\frac{1}{2}}}{2 \sinh \left(2 \pi \mathrm{ie}^{\theta}\right)} \\
& \times\left[\zeta_{+}\left(\theta-\mathrm{i} \pi\left(j+\frac{1}{2}\right)\right) \zeta_{-}\left(\theta+\mathrm{i} \pi\left(j+\frac{1}{2}\right)\right)-\zeta_{-}\left(\theta-\mathrm{i} \pi\left(j+\frac{1}{2}\right)\right) \zeta_{+}\left(\theta+\mathrm{i} \pi\left(j+\frac{1}{2}\right)\right)\right]
\end{aligned}
$$

whereas for $j=0,1, \ldots$

$$
\begin{aligned}
& \tau_{j}(\lambda)=\frac{(-1)^{j}}{2 \sinh \left(2 \pi \mathrm{e}^{\theta}\right)} \\
& \times\left[\zeta_{+}\left(\theta-\mathrm{i} \pi\left(j+\frac{1}{2}\right)\right) \zeta_{+}\left(\theta+\mathrm{i} \pi\left(j+\frac{1}{2}\right)\right)-\zeta_{-}\left(\theta-\mathrm{i} \pi\left(j+\frac{1}{2}\right)\right) \zeta_{-}\left(\theta+\mathrm{i} \pi\left(j+\frac{1}{2}\right)\right)\right] .
\end{aligned}
$$

where $\lambda$ and $\theta$ are related as in eq. (4.5). With these formulae the fusion relation (2.41), as well as the $T-Q$ type relations (4.6), are satisfied identically.

Finally, let us consider the " $\mu \rightarrow-\mu$ symmetry" relations (v). It is easy to see that in the scaling limit they become

$$
\zeta_{ \pm}(\theta+\mathrm{i} \pi n)=(-1)^{\mathfrak{m}} \mathrm{e}^{ \pm 2 \pi \mathrm{ie}^{\theta}} \zeta_{\mp}(\theta) \quad(n-\text { odd })
$$

and

$$
\begin{array}{ll}
\zeta_{+}(\theta+\mathrm{i} \pi n)=(-1)^{\mathfrak{m}} \zeta_{+}(\theta) & (n-\text { even }) \\
\zeta_{-}(\theta+\mathrm{i} \pi n) & =(-1)^{\mathfrak{m}} \mathrm{e}^{4 \pi \mathrm{ie}^{\theta}} \zeta_{-}(\theta) .
\end{array}
$$

As we will see below, these equations are not satisfied for non-integer $n$ and only hold for the admissible values of $\mathfrak{j}$ and $\mathfrak{m}$ (3.14). In these particular cases, they can be used to truncate the so called $Y$-system - the chain of relations for $Y_{j}(\lambda) \equiv \tau_{j-\frac{1}{2}}(\lambda) \tau_{j+\frac{1}{2}}(\lambda)$ (see, e.g., refs. $[11,19])$. In turn, the truncated $Y$-system allows one to derive the set of TBA equations from which the eigenvalues of the chiral transfer-matrices can be computed. For the vacuum eigenvalues with $\mathfrak{j}=\mathfrak{m}=0$, the TBA system can be found in ref. [43].

\subsection{ODE/IQFT correspondence for the vacuum eigenvalues}

Let us first consider the vacuum eigenvalues $\zeta_{ \pm}^{(\mathrm{vac})}(\theta)$ of the operators $\zeta_{ \pm}(\theta)$ corresponding to the real pair $\left(p_{1}, p_{2}\right)$. The main ingredient in the ODE/IQFT correspondence for this case, is the ordinary differential equation

$$
\left[-\frac{\mathrm{d}^{2}}{\mathrm{~d} x^{2}}-p_{1}^{2} \frac{\mathrm{e}^{x}}{1+\mathrm{e}^{x}}+\left(\frac{1}{4}-p_{2}^{2}\right) \frac{\mathrm{e}^{x}}{\left(1+\mathrm{e}^{x}\right)^{2}}+\mathrm{e}^{2 \theta}\left(1+\mathrm{e}^{x}\right)^{n}\right] \Psi(x)=0 .
$$

We assume for the moment that $\theta$ is real. Eq. (4.17) has the form of a stationary zero energy Schrödinger equation with the potential $V(x)$ given by the last three terms in (4.17). The 
potential $V(x)$ is positive and grows fast at large positive $x$ so that (4.17) has a solution $\Xi(x)$ decaying at $x \rightarrow+\infty$; this condition specifies $\Xi(x)$ uniquely up to normalization. To fix the normalization, we assume that

$$
\Xi(x) \rightarrow \mathrm{e}^{-\frac{\theta}{2}} \exp \left(-\left(\frac{n}{4}+\mathrm{e}^{\theta}\right) x-\mathrm{e}^{\theta} \int_{0}^{\mathrm{e}^{x}} \frac{\mathrm{d} u}{u}\left((1+u)^{\frac{n}{2}}-1\right)\right)
$$

as $x \rightarrow+\infty$. On the other hand, $V(x)$ approaches the positive constant $\mathrm{e}^{2 \theta}$ at large negative $x$. Hence eq. (4.17) has a solution which decays for large negative $x$; we denote this solution $\Psi_{+}(x)$. The condition,

$$
\Psi_{+}(x) \rightarrow \sqrt{\pi} \frac{\exp \left(\mathrm{e}^{\theta} x\right)}{\Gamma\left(1+2 \mathrm{e}^{\theta}\right)} \quad \text { as } \quad x \rightarrow-\infty,
$$

specifies the solution $\Psi_{+}(x)$ uniquely, including its normalization. Then

$$
\zeta_{+}^{(\mathrm{vac})}(\theta)=\exp \left(C_{+} \mathrm{e}^{\theta}\right) W\left[\Xi, \Psi_{+}\right]
$$

where

$$
C_{+}=-\pi \cot \left(\frac{\pi n}{2}\right)+2 \log (2)-2
$$

and $W[f, g]$ denotes the Wronskian $f(x) g^{\prime}(x)-g(x) f^{\prime}(x)$. Eq. (4.20) was proposed in the work [42].

In the paper [43] this was extended to the vacuum eigenvalue $\zeta_{-}^{(\mathrm{vac})}(\theta)$. The starting point was the same differential equation (4.17), but instead of $\Psi_{+}$in (4.20), another solution which grows as $\exp \left(-\mathrm{e}^{\theta} x\right)$ at large negative $x$ was taken. Of course, this condition alone does not define the solution uniquely since, besides the overall normalization, one can always add any amount of $\Psi_{+}(x)$. It is usually difficult to define a growing solution unambiguously, but in our case the following property of (4.17) helps. Let us consider $x$ as a complex variable. The potential $V(x)$ is an analytic function of $x$ with branch-point singularities at all points where $\mathrm{e}^{x}$ turns to -1 . Let us make branch cuts starting at each of the points $x=\mathrm{i} \pi(2 N+1), N=0, \pm 1, \pm 2, \ldots$ and going to $+\infty$ parallel to the real axis, and choose the branch of $V(x)$ for which $\left(1+\mathrm{e}^{x}\right)^{n}$ is real and positive on the real axis of the $x$-plane. Restricting attention to the domain $\Re e(x)<0$, one finds that the potential $V(x)$ has the periodicity property

$$
V(x+2 \pi \mathrm{i})=V(x) \quad(\Re e(x)<0) .
$$

Consequently, equation (4.17) has two Bloch-wave solutions $\left(2 \mathrm{e}^{\theta} \notin \mathbb{Z}\right)$ :

$$
\Psi_{ \pm}(x+2 \pi \mathrm{i})=\mathrm{e}^{ \pm 2 \pi \mathrm{i} \mathrm{e}^{\theta}} \Psi_{ \pm}(x) \quad(\Re e(x)<0)
$$

where the Bloch factors can be found by taking the limit $\Re e(x) \rightarrow-\infty$. At this point we assume that $2 \mathrm{e}^{\theta}$ is not an integer, so that the conditions (4.23) specify two independent solutions $\Psi_{ \pm}(x)$ uniquely, up to their normalizations. Of course, the solution $\Psi_{+}(x)$ defined this way decays as $\exp \left(\mathrm{e}^{\theta} x\right)$ at $\Re e(x) \rightarrow-\infty$, and the asymptotic condition (4.19) also fixes 
its normalization. The solution $\Psi_{-}(x)$ grows at large negative $\Re e(x)$, and its normalization can be fixed by specifying the leading asymptotic in this domain. Thus we define $\Psi_{-}(x)$ by the conditions

$$
\begin{aligned}
& \Psi_{-}(x+2 \pi \mathrm{i})=\mathrm{e}^{-2 \pi \mathrm{e}^{\theta}} \Psi_{-}(x) \\
& (\Re e(x)<0) \\
& \Psi_{-}(x) \rightarrow \sqrt{\pi} \frac{\exp \left(-\mathrm{e}^{\theta} x\right)}{\Gamma\left(1-2 \mathrm{e}^{\theta}\right)} \quad \text { as } \quad \Re e(x) \rightarrow-\infty .
\end{aligned}
$$

It is possible to show that both $\Psi_{+}(x)$ and $\Psi_{-}(x)$ defined by (4.19) and (4.24) are entire functions of $\mathrm{e}^{\theta}$, and

$$
\Psi_{-}\left(x \mid \mathrm{e}^{\theta}\right)=\Psi_{+}\left(x \mid-\mathrm{e}^{\theta}\right),
$$

where we temporarily exhibited the dependence of $\Psi_{ \pm}$on the parameter $\mathrm{e}^{\theta}$. From the definitions (4.19) and (4.24) we have

$$
W\left[\Psi_{-}, \Psi_{+}\right]=\sin \left(2 \pi \mathrm{e}^{\theta}\right) .
$$

The proposal in [43] was that

$$
\zeta_{-}^{(\mathrm{vac})}(\theta)=\exp \left(C_{-} \mathrm{e}^{\theta}\right) W\left[\Xi, \Psi_{-}\right]
$$

where

$$
C_{-}=-\pi \cot \left(\frac{\pi n}{2}\right)-2 \log (2)+2-2 \gamma_{E}-2 \psi\left(1+\frac{n}{2}\right)
$$

with $\psi(z)=\partial_{z} \log \Gamma(z)$ and $\gamma_{E}$ stands for the Euler constant.

There is much evidence to support the remarkable relations (4.20) and (4.27). Some of them are based on WKB analysis of the differential equation (4.17). Using the method of semiclassical expansion, one can systematically study the Wronskians in (4.20), (4.27) at $\theta \rightarrow+\infty$. This yields asymptotic expansions whose structures turn out to be identical to the one for $\zeta_{ \pm}^{(\mathrm{vac})}$ following from eq. (4.9). Furthermore the WKB calculations give nontrivial predictions for the vacuum eigenvalues of the local and dual nonlocal IM. On the other hand, these vacuum eigenvalues can be directly calculated from their definitions. For example, the vacuum eigenvalue of the first dual nonlocal IM is given by eq. (2.49), while it is a simple exercise to find the vacuum eigenvalue of the first local IM $\mathfrak{i}_{1}$ :

$$
i_{1}\left(p_{1}, p_{2}\right)=-\frac{1}{12}+\frac{p_{1}^{2}}{n}+\frac{p_{2}^{2}}{n+2} .
$$

It turns out that the results of the WKB analysis are in full agreement with these direct calculations.

Let us discuss now the $\theta \rightarrow-\infty$ form of the Wronskians in (4.20) and (4.27). The perturbative evaluation of the solutions $\Xi(x)$ and $\Psi_{ \pm}(x)$ leads to the expansions $[42,43]$

$$
\mathrm{e}^{ \pm \pi \kappa \cot \left(\frac{\pi n}{2}\right)} \zeta_{ \pm}^{(\mathrm{vac})}(\theta)=B_{p_{1}, p_{2}} \mathrm{e}^{\frac{2 \mathrm{i} p_{1} \theta}{n}} F_{ \pm}\left(\theta \mid p_{1}, p_{2}\right)+B_{-p_{1}, p_{2}} \mathrm{e}^{-\frac{2 \mathrm{i} p_{1} \theta}{n}} F_{ \pm}\left(\theta \mid-p_{1}, p_{2}\right)
$$

where

$$
B_{p_{1}, p_{2}}=\sqrt{n} n^{-\frac{2 \mathrm{i} p_{1}}{n}} \frac{\Gamma\left(-2 \mathrm{i} p_{1}\right) \Gamma\left(1-\frac{2 \mathrm{i} p_{1}}{n}\right)}{\Gamma\left(\frac{1}{2}-p_{2}-\mathrm{i} p_{1}\right) \Gamma\left(\frac{1}{2}+p_{2}-\mathrm{i} p_{1}\right)}
$$


and

$$
F_{ \pm}\left(\theta \mid p_{1}, p_{2}\right)=\sum_{i, j=0}^{\infty} f_{i, j}\left(p_{1}, p_{2}\right)( \pm 1)^{i} \mathrm{e}^{\left(i+\frac{2 j}{n}\right) \theta}
$$

with

$$
\begin{aligned}
& f_{0,0}=1 \\
& f_{0,1}=-\frac{\Gamma\left(\frac{1}{2}+\frac{1}{n}\right) \Gamma\left(1-\frac{1}{n}\right)}{2 \sqrt{\pi}}\left(\frac{2}{n}\right)^{\frac{2}{n}-1}\left(\frac{n+2}{n-2}+\frac{4 p_{2}^{2}}{1+4 p_{1}^{2}}\right) \frac{\Gamma\left(1-\frac{1}{n}-\frac{2 \mathrm{i} p_{1}}{n}\right)}{\Gamma\left(\frac{1-2 \mathrm{i} p_{1}}{n}\right)} \\
& f_{1,0}=-\psi\left(\frac{1}{2}-p_{2}-\mathrm{i} p_{1}\right)-\psi\left(\frac{1}{2}+p_{2}-\mathrm{i} p_{1}\right)+\psi\left(-\frac{n}{2}\right)+\gamma_{E}+2 \log (2)-2 .
\end{aligned}
$$

Note that the integer powers of $\mathrm{e}^{\theta}$ in (4.32) come from the perturbative expansion of the solution $\Psi_{ \pm}(x)$. In view of $(4.25)$, these powers in $F_{+}$are related to the corresponding powers in $F_{-}$by a change of sign, $\mathrm{e}^{\theta} \rightarrow-\mathrm{e}^{\theta}$. At the same time, the powers of $\mathrm{e}^{\frac{2 \theta}{n}}$ are the result of the expansion of $\Xi(x)$, and hence they are the same in $F_{-}$and $F_{+}$.

One of the important properties of the Wronskians on the right hand side of eqs. (4.20), (4.27), is that for a given $\theta$, they are entire functions in both complex variables $p_{1}^{2}$ and $p_{2}^{2}$. Thus it is perfectly fine to consider them for pure imaginary values of $p_{1}$. Let's set $p_{1}=\frac{i}{2} \mathfrak{m}, p_{2}=\mathfrak{j}+\frac{1}{2}$ and assume that $\mathfrak{m} \geq 0$. In this notation, the coefficient $B_{p_{1}, p_{2}}$ (4.31) contains the factor $\Gamma\left(-\mathfrak{j}+\frac{\mathfrak{m}}{2}\right)$ in the denominator, and therefore vanishes when $\mathfrak{j}-\frac{\mathfrak{m}}{2}$ is a non-negative integer. At the same time, the coefficient $B_{-p_{1}, p_{2}}$ takes the form $B_{-p_{1}, p_{2}}=B_{s}(\mathfrak{m})$ with

$$
B_{s}(\mathfrak{m})=\sqrt{n} n^{-\frac{\mathfrak{m}}{n}} \Gamma\left(1-\frac{\mathfrak{m}}{n}\right) \frac{(-1)^{s}}{s !} \frac{\Gamma(1+\mathfrak{m}+s)}{\Gamma(1+\mathfrak{m})} \quad\left(s=\mathfrak{j}-\frac{1}{2} \mathfrak{m}=0,1,2, \ldots\right)
$$

which remains finite for the discrete set of $\mathfrak{j}$ and $\mathfrak{m}$ (3.14) corresponding to the bosonization of the parafermionic spaces $\mathcal{V}_{j}^{(\mathfrak{m})}$. The vanishing of the coefficient $B_{p_{1}, p_{2}}$ does not actually mean that we can neglect the first term in the sum (4.30) - the expansion coefficients in $F_{ \pm}\left(\theta \mid p_{1}, p_{2}\right)$ may become singular for $\left(p_{1}, p_{2}\right)$ given by eqs. (3.10), (3.14). Nevertheless, analysis shows that such "resonances" occur only for the terms $\propto \mathrm{e}^{\left(-\frac{\mathfrak{m}}{n}+i+\frac{2 j}{n}\right) \theta}$ with $i \geq 0$ and $j \geq \mathfrak{m}$, i.e., having the same form as monomials in the double sum $\mathrm{e}^{\frac{\mathfrak{m}}{n}} \theta F_{ \pm}\left(\theta \mid-p_{1}, p_{2}\right)$. Notice that for integer $n$, the double summations in $F_{ \pm}$can be replaced by a single one. This yields the formulae for the regularized (see eq. (4.11)) vacuum eigenvalues in the parafermionic spaces $\mathcal{V}_{j}^{(\mathfrak{m})}$ :

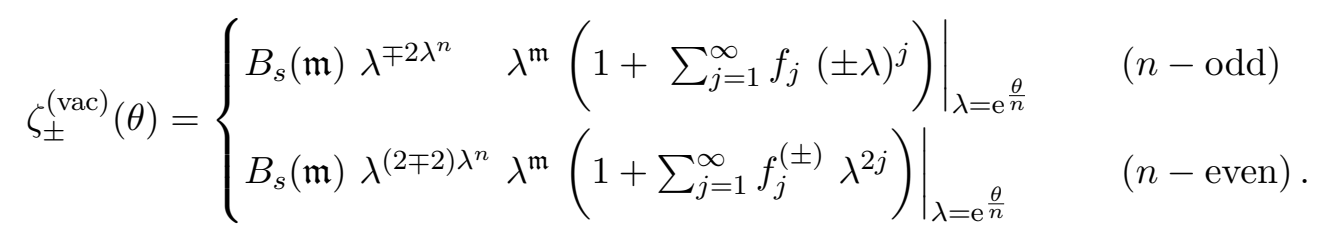

The appearance of the "strange" factors, $\lambda^{\mp 2 \lambda^{n}}, \lambda^{(2 \mp 2) \lambda^{n}}$ here can be understood as follows. The Wronskians in the l.h.s. of eqs. (4.20) and (4.27) are, of course, non-singular functions 
of $\left(p_{1}, p_{2}\right)$ and $n>0 .{ }^{10}$ However, the individual coefficients $f_{i, j}$ in (4.32) become singular for some values of the parameters, as it can be seen from the explicit formulae (4.33). When the values of the parameters are restricted to the parafermionic case, the singularities from the different coefficients must cancel each other giving rise to terms $\propto \theta^{m} \mathrm{e}^{l \theta}$ which sum up to yield the "strange" factors in (4.35). Notice also that some of the coefficients in these series expansions are known explicitly. In appendix B their values are compared with the corresponding results obtained from the vacuum eigenvalues of the finite matrices $\mathcal{Z}_{ \pm}(\mu)$.

We now return to the differential equation and consider the solution $\Xi$ in more detail. For complex $\theta$, the asymptotic condition (4.18) unambiguously defines $\Xi(x \mid \theta)$ in the strip $|\Im m(\theta)| \leq \frac{\pi}{2}$ including its boundary. The two functions $\Xi\left(x \mid \theta+\frac{\mathrm{i} \pi}{2}\right)$ and $\Xi\left(x \mid \theta-\frac{\mathrm{i} \pi}{2}\right)$, with real $\theta$, form a linear basis in the space of solutions of (4.17) with $\mathrm{e}^{2 \theta}$ substituted by $\left(-\mathrm{e}^{2 \theta}\right)$, since as it follows from (4.18),

$$
W\left[\Xi\left(x \mid \theta+\frac{\mathrm{i} \pi}{2}\right), \Xi\left(x \mid \theta-\frac{\mathrm{i} \pi}{2}\right)\right]=2 \mathrm{i} .
$$

On the other hand, formulae (4.20), (4.27) and (4.26) imply

$$
\Xi(x \mid \theta)=\frac{\exp \left(\xi \mathrm{e}^{\theta}\right)}{\sin \left(2 \pi \mathrm{e}^{\theta}\right)}\left(\zeta_{+}^{(\mathrm{vac})}(\theta) \mathrm{e}^{-c \mathrm{e}^{\theta}} \Psi_{+}\left(x \mid-\mathrm{e}^{\theta}\right)-\zeta_{-}^{(\mathrm{vac})}(\theta) \mathrm{e}^{+c \mathrm{e}^{\theta}} \Psi_{+}\left(x \mid+\mathrm{e}^{\theta}\right)\right)
$$

which can be used to express $\Xi\left(x \mid \theta \pm \frac{\mathrm{i} \pi}{2}\right)$ in terms of $\Psi_{+}\left(x \mid \pm \mathrm{ie}^{\theta}\right)$ and then calculate the l.h.s. of (4.36). This yields the quantum Wronskian type relation (4.4) specified at the vacuum eigenvalues of $\zeta_{ \pm}(\theta)$. Notice that in eq. (4.37) we use the constants $\xi=-\frac{1}{2}\left(C_{+}+C_{-}\right)$ and $c=\frac{1}{2}\left(C_{+}-C_{-}\right)$. Of course, the factors $\exp \left( \pm c \mathrm{e}^{\theta}\right)$ which appear in this formula can be included in the definition of the solutions $\Psi_{ \pm}$. With the value of $c$ determined by eqs. (4.21), (4.28), the constant $C$, appearing in the asymptotic expansions (4.9) for $\zeta_{ \pm}(\theta)$, vanishes. The constant

$$
\xi=-\frac{1}{2}\left(C_{+}+C_{-}\right)=\pi \cot \left(\frac{\pi n}{2}\right)+\gamma_{E}+\psi\left(1+\frac{n}{2}\right)
$$

can also be absorbed into the definition of the solution $\Xi(x)$.

Let us assume that the solution $\Xi(x \mid \theta)$ can be unambiguously continued to the whole complex plane from the strip $|\Im m(\theta)| \leq \frac{\pi}{2} .{ }^{11}$ Then the function $\Xi\left(x \mid \theta+\mathrm{i} \pi\left(2 j+\frac{1}{2}\right)\right)$ with $j=0, \frac{1}{2}, 1, \ldots$ solves the differential equation (4.17) with $\mathrm{e}^{2 \theta} \mapsto\left(-\mathrm{e}^{2 \theta}\right)$ and can be linearly expressed in terms of the two basic solutions $\Xi\left(x \mid \theta \pm \frac{\mathrm{i} \pi}{2}\right)$

$$
\Xi\left(x \mid \theta+\mathrm{i} \pi\left(2 j+\frac{1}{2}\right)\right)=a_{j}(\theta) \Xi\left(x \mid \theta-\frac{\mathrm{i} \pi}{2}\right)+b_{j}(\theta) \Xi\left(x \mid \theta+\frac{\mathrm{i} \pi}{2}\right) .
$$

With manipulations similar to those which lead to the quantum Wronskian type relations, it is straightforward to show that

$$
a_{j}(\theta)=-b_{j-\frac{1}{2}}(\theta+\mathrm{i} \pi)
$$

\footnotetext{
${ }^{10}$ The only singularities at $n=2,4, \ldots$ are produced by the cotangent which shows up in the expression for the constants $C_{ \pm}(4.21),(4.28)$. The reason that this term was included in the definition of $\zeta_{ \pm}$is that we would like the $T-Q$ type relations to have the simple canonical form (4.6).

${ }^{11}$ It is expected that $\Xi(x \mid \theta)$ is an entire function of $\theta$. However at this moment, we don't have a rigorous proof of this statement.
} 
and the $b_{j}(\theta-\mathrm{i} \pi j)$ are given by the r.h.s. of (4.13), (4.14) where the operators $\zeta_{ \pm}$are substituted by their vacuum eigenvalues. Hence we conclude that

$$
\tau_{j}^{(\mathrm{vac})}(\theta)=b_{j}(\theta-\mathrm{i} \pi j) .
$$

\subsection{ODE/IQFT correspondence for the full spectrum}

In the previous subsection, our analysis was restricted to the vacuum eigenvalues. As a matter of fact, along the line of ref. [25], it can be extended to the whole spectrum of the commuting families of operators. This was already done for a more general case in the work [58]. Here we give a sketch of the construction.

Let $z$ be the complex coordinate on $\mathbb{C P}^{1} \backslash\left\{z_{1}, z_{2}, z_{3}\right\}$, the Riemann sphere with three punctures. Consider the second order Fuchsian differential operator $-\partial_{z}^{2}+T_{0}(z)$, with $T_{0}(z)$ given by

$$
T_{0}(z)=-\sum_{i=1}^{3}\left(\frac{\delta_{i}}{\left(z-z_{i}\right)^{2}}+\frac{c_{i}}{z-z_{i}}\right)
$$

and the $\delta_{i}$ are regarded as independent parameters, whereas the $c_{i}$ are unambiguously defined by the constraints

$$
\sum_{i=1}^{3} c_{i}=0, \quad \sum_{i=1}^{3}\left(z_{i} c_{i}+\delta_{i}\right)=0, \quad \sum_{i=1}^{3}\left(z_{i}^{2} c_{i}+2 z_{i} \delta_{i}\right)=0 .
$$

The equation

$$
\left(-\partial_{z}^{2}+T_{0}(z)\right) \psi=0
$$

is a second-order differential equation with three regular singular points. For a generic choice of the parameters $\delta_{i}$, it can be brought to the standard hypergeometric form by a change of variables. In the papers [57], a deformation of (4.44) was introduced, of the form

$$
\mathcal{D}_{0}(\theta) \psi=0, \quad \mathcal{D}_{0}(\theta)=-\partial_{z}^{2}+T_{0}(z)+\mathrm{e}^{2 \theta} \mathcal{P}(z),
$$

where $\theta$ stands for an arbitrary complex parameter and

$$
\mathcal{P}(z)=\frac{\left(z_{3}-z_{2}\right)^{a_{1}}\left(z_{3}-z_{1}\right)^{a_{2}}\left(z_{2}-z_{1}\right)^{a_{3}}}{\left(z-z_{1}\right)^{2-a_{1}}\left(z-z_{2}\right)^{2-a_{2}}\left(z-z_{3}\right)^{2-a_{3}}}
$$

with

$$
a_{1}+a_{2}+a_{3}=0 .
$$

Notice that, because of the last relation, $\mathcal{P}(z)(\mathrm{d} z)^{2}$ transforms as a quadratic differential under the $\mathbb{P S L}(2, \mathbb{C})$ group, so that the punctures $z_{1}, z_{2}, z_{3}$ on the Riemann sphere can be sent to any desirable positions.

The immediate object of our interest is a particular case of the differential equation (4.45) with

$$
a_{1}=-n, \quad a_{2}=n+2, \quad a_{3}=0
$$

and

$$
\delta_{1}=\frac{1}{4}+p_{1}^{2}, \quad \delta_{2}=\frac{1}{4}-p_{2}^{2}, \quad \delta_{3}=\frac{1}{4} .
$$


Indeed, the change of variables

$$
\mathrm{e}^{x}=\frac{z-z_{3}}{z-z_{1}} \frac{z_{2}-z_{1}}{z_{3}-z_{2}}, \quad \Psi(x)(\mathrm{d} x)^{-\frac{1}{2}}=\psi(z)(\mathrm{d} z)^{-\frac{1}{2}},
$$

brings equation (4.17) to the form (4.45) specialized to this set of parameters.

As it was explained in [58], the description of the spectrum of the commuting family of transfer-matrices in the Fock level subspaces $\mathcal{F}_{p_{1}, p_{2}}^{(L)}$ is based on a differential equation of the form similar to (4.45), where the differential operator $\mathcal{D}_{0}(\theta)$ is substituted by

$$
\mathcal{D}(\theta)=-\partial_{z}^{2}+T_{L}(z)+\mathrm{e}^{2 \theta} \mathcal{P}(z),
$$

which has $3+L$ singular points at $z=z_{1}, z_{2}, z_{3}$ and also $z=x_{1}, \ldots, x_{L}$. The form of $T_{L}(z)$, including the positions of the extra singularities, $z=x_{1}, \ldots, x_{L}$, is determined by the requirement that the monodromy properties of the solutions to the equation $\mathcal{D}(\theta) \psi(z)=0$ are identical to those for $\mathcal{D}_{0}(\theta) \psi(z)=0$. It turns out that this requirement can be fulfilled if the set of complex numbers $\left\{x_{i}\right\}_{i=1}^{L}$ obey a system of $L$ algebraic equations similar to that from $[25,59,60]$.

As we saw in the previous subsection, the vacuum eigenvalues of the operators $\zeta_{ \pm}(\theta)$ and $\tau(\theta)$ can be identified with certain connection coefficients for the differential equation (4.17), or equivalently for (4.45)-(4.49). ${ }^{12}$ Of course, similar connection coefficients can be associated to the more general differential operator (4.51). Since the singularities of the potential $T_{L}(z)$ at $z=x_{1}, \ldots, x_{L}$ do not affect the monodromy properties of the solutions of $\mathcal{D}(\theta) \psi(z)=0$, all the relations between the connection coefficients remain unchanged. This allows one to identify them with specializations of the operator relations like (4.4), (4.6), (4.13), (4.14) and (4.9), to the eigenvalues of the commuting families of operators.

\subsection{Operators $\beta_{ \pm}(\theta)$ and $\alpha_{ \pm}(\theta)$}

With the philosophy of the ODE/IQFT correspondence in mind, let us return to the differential equation (4.17). It has three singular points at $\mathrm{e}^{x}=0, \infty,-1$, and we have already discussed the canonical bases in the space of solutions in the neighbourhood of two of them, $\mathrm{e}^{x}=0$ and $\infty$. We now consider the basis which is canonically defined in the vicinity of $\mathrm{e}^{x}=-1$. For this purpose it is convenient to perform a change of variables

$$
\mathrm{e}^{-x}=-1-\mathrm{e}^{-y}, \quad \Psi(x)=\left(1+\mathrm{e}^{y}\right)^{-\frac{1}{2}} \tilde{\Psi}(y),
$$

which brings (4.17) to the form

$$
\left[-\frac{\mathrm{d}^{2}}{\mathrm{~d} y^{2}}+p_{2}^{2} \frac{\mathrm{e}^{y}}{1+\mathrm{e}^{y}}+\left(\frac{1}{4}+p_{1}^{2}\right) \frac{\mathrm{e}^{y}}{\left(1+\mathrm{e}^{y}\right)^{2}}+\mathrm{e}^{2 \theta}\left(1+\mathrm{e}^{y}\right)^{-n-2}\right] \tilde{\Psi}(y)=0 .
$$

For $p_{2}>0$ the differential equation (4.53) admits a unique solution such that

$$
\tilde{\Theta}_{+}(y) \rightarrow \sqrt{\frac{\pi}{n+2}}(n+2)^{-\frac{2 p_{2}}{n+2}} \frac{\mathrm{e}^{-p_{2} y}}{\Gamma\left(1+\frac{2 p_{2}}{n+2}\right)} \quad \text { as } y \rightarrow+\infty .
$$

\footnotetext{
${ }^{12}$ By "connection coefficients" we understand the $\theta$-dependent functions which allow one to relate different bases in the linear space of solutions of the ordinary differential equation, see eqs. (4.37) and (4.39).
} 
For complex $p_{2}$, one can show that the solution is a meromorphic function, analytic in the half plane $\Re e\left(p_{2}\right) \geq 0$. Since the equation (4.53) is invariant w.r.t. the substitution $p_{2} \mapsto-p_{2}$, for generic values of $p_{2}$ one can define the second linear independent solution uniquely by analytic continuation $\mathscr{A}_{p_{2} \mapsto \mathrm{e}^{ \pm \mathrm{i} \pi} p_{2}}$ to the half plane $\Re e\left(p_{2}\right)<0$ :

$$
\tilde{\Theta}_{-}\left(y \mid \mathrm{e}^{2 \theta}, p_{2}\right)=\mathscr{A}_{p_{2} \mapsto \mathrm{e}^{ \pm \mathrm{i} \pi} p_{2}}\left[\tilde{\Theta}_{+}\left(y \mid \mathrm{e}^{2 \theta}, p_{2}\right)\right]
$$

and it is easy to see that

$$
W\left[\tilde{\Theta}_{+}, \tilde{\Theta}_{-}\right]=\sin \left(\frac{2 \pi p_{2}}{n+2}\right) .
$$

Notice that both these solutions are entire functions of the variable $\mathrm{e}^{2 \theta}$ whose dependence is emphasized in the formula (4.55).

As $y \rightarrow-\infty$, eq. (4.53) admits the two linearly independent solutions

$$
\tilde{\Psi}_{ \pm}(y) \rightarrow \sqrt{\pi} \frac{\exp \left( \pm \mathrm{e}^{\theta} y\right)}{\Gamma\left(1 \pm 2 \mathrm{e}^{\theta}\right)} \quad \text { as } \quad y \rightarrow-\infty .
$$

Of course, $\tilde{\Psi}_{ \pm}(y)$ are obtained from $\Psi_{ \pm}(x)(4.19),(4.24)$ by means of the coordinate transformation (4.52):

$$
\Psi_{ \pm}(x)(\mathrm{d} x)^{-\frac{1}{2}}=\exp \left(\mp \mathrm{i} \pi \mathrm{e}^{\theta}\right) \quad \tilde{\Psi}_{ \pm}(y)(\mathrm{d} y)^{-\frac{1}{2}}
$$

Here the extra phase factor appears because of slightly different normalizations: $\Psi_{ \pm}(x)$ were defined in such a way that they stay real for real $x$ and real values of the parameters, whereas $\tilde{\Psi}_{ \pm}(y)(4.57)$ are real for real $y$ which is related to $x$ as $\mathrm{e}^{-y}=-1-\mathrm{e}^{-x}$. It is important to keep in mind the presence of such phase factors, because they affect the reality conditions for the connection coefficients.

Following the philosophy of the ODE/IQFT correspondence, we consider the connection coefficients for the bases $\left\{\tilde{\Psi}_{ \pm}(y)\right\}$ and $\left\{\tilde{\Theta}_{ \pm}(y)\right\}$ and interpret them as vacuum eigenvalues of certain operators $\beta_{ \pm}(\theta)$ :

$$
\mathrm{e}^{+c \mathrm{e}^{\theta}} \tilde{\Psi}_{+}\left(y \mid \mathrm{e}^{\theta}\right)=\frac{1}{2 \sin \left(\frac{2 \pi p_{2}}{n+2}\right)}\left(\beta_{+}^{(\mathrm{vac})}(\theta) \tilde{\Theta}_{-}\left(y \mid \mathrm{e}^{2 \theta}\right)-\beta_{-}^{(\mathrm{vac})}(\theta) \tilde{\Theta}_{+}\left(y \mid \mathrm{e}^{2 \theta}\right)\right)
$$

The relation for $\mathrm{e}^{-c \mathrm{e}^{\theta}} \tilde{\Psi}_{-}$is similar with $\beta_{ \pm}^{(\mathrm{vac})}(\theta)$ substituted by $\beta_{ \pm}^{(\mathrm{vac})}(\theta+\mathrm{i} \pi)$. Notice that it is expected that

$$
\beta_{ \pm}(\theta+\mathrm{i} \pi)=\beta_{ \pm}(\theta-\mathrm{i} \pi) .
$$

Further, the operators $\beta_{ \pm}$are entire functions of the variable $\mathrm{e}^{\theta}$ and can be written in the form of a convergent series

$$
\beta_{ \pm}(\theta)=b_{ \pm}\left(1+\sum_{m=1}^{\infty} \mathfrak{b}_{m}^{( \pm)} \mathrm{e}^{m \theta}\right) .
$$

The reality condition for the connection coefficients $\beta_{ \pm}^{(\mathrm{vac})}(\theta)$ suggests the Hermiticity

$$
\left[\beta_{ \pm}(\theta)\right]^{\dagger}=\beta_{ \pm}\left(\theta^{*}\right)
$$


Using the definition (4.59), one can express the Wronskian $W\left[\tilde{\Psi}_{-}, \tilde{\Psi}_{+}\right]$in terms of the connection coefficients $\beta_{ \pm}^{\text {(vac) }}$. On the other hand, as follows from (4.57), it is equal to $\sin \left(2 \pi \mathrm{e}^{\theta}\right)$. This yields the quantum Wronskian relation

$$
\beta_{+}\left(\theta+\frac{\mathrm{i} \pi}{2}\right) \beta_{-}\left(\theta-\frac{\mathrm{i} \pi}{2}\right)-\beta_{-}\left(\theta+\frac{\mathrm{i} \pi}{2}\right) \beta_{+}\left(\theta-\frac{\mathrm{i} \pi}{2}\right)=-4 \mathrm{i} \sinh \left(2 \pi \mathrm{e}^{\theta}\right) \sin \left(\frac{2 \pi p_{2}}{n+2}\right),
$$

where the superscript "(vac)" is omitted, since we expect that it holds true for all eigenvalues of the operators $\beta_{ \pm}$.

It turns out that the operators (4.61), acting in the Fock space $\mathcal{F}_{p_{1}, p_{2}}$, and satisfying the commutativity conditions

$$
\left[\beta_{\sigma}(\theta), \beta_{\sigma^{\prime}}\left(\theta^{\prime}\right)\right]=\left[\beta_{\sigma}(\theta), \tau\left(\theta^{\prime}\right)\right]=0 \quad\left(\sigma, \sigma^{\prime}= \pm\right),
$$

can be defined explicitly. Their construction lies beyond the scope of this work. Here we just mention that it is similar to the construction of the $U_{q}\left(\hat{\mathfrak{s l}}_{2}\right) Q$-operators from refs. [18, 19].

The last set of connection coefficients relates the basis in the space of solutions canonically defined at the singular point $\mathrm{e}^{x}=\infty$ with that defined at $\mathrm{e}^{x}=-1$, or, equivalently $\mathrm{e}^{y}=-1$ with $\mathrm{e}^{y}=0$. Let $\left.\tilde{\Xi}(y) \equiv\left(1+\mathrm{e}^{y}\right)^{\frac{1}{2}} \Xi(x)\right|_{x(y)}$. Then it can be written in the form similar to (4.37) and (4.59):

$$
\tilde{\Xi}(y \mid \theta)=\frac{\exp \left(\xi \mathrm{e}^{\theta}\right)}{\sin \left(\frac{2 \pi p_{2}}{n+2}\right)}\left(a_{+}(\theta) \tilde{\Theta}_{-}\left(y \mid \mathrm{e}^{2 \theta}\right)-a_{-}(\theta) \tilde{\Theta}_{+}\left(y \mid \mathrm{e}^{2 \theta}\right)\right) .
$$

Since for real $y$, the solution $\tilde{\Xi}(y)$ is complex, the reality condition looks simpler if we introduce $\alpha_{ \pm}^{(\mathrm{vac})}(\theta)$, such that

$$
a_{ \pm}(\theta)=\mathrm{i} \mathrm{e}^{\mp \mathrm{i} \pi p_{2}} \alpha_{ \pm}^{(\mathrm{vac})}\left(\theta-\frac{\mathrm{i} \pi n}{2}\right)
$$

The latter turn out to be real functions for real $\theta$ and $\left(p_{1}, p_{2}\right)$. Again, we interpret them as the vacuum eigenvalues of the Hermitian operators $\alpha_{ \pm}(\theta)$,

$$
\left[\alpha_{ \pm}(\theta)\right]^{\dagger}=\alpha_{ \pm}\left(\theta^{*}\right)
$$

which are also expected to be entire functions of $\theta$. Similar to the operators $\zeta_{ \pm}(\theta)$, one can obtain the quantum Wronskian relation

$$
\alpha_{+}\left(\theta-\frac{\mathrm{i} \pi}{2}\right) \alpha_{-}\left(\theta+\frac{\mathrm{i} \pi}{2}\right)-\alpha_{-}\left(\theta-\frac{\mathrm{i} \pi}{2}\right) \alpha_{+}\left(\theta+\frac{\mathrm{i} \pi}{2}\right)=2 \mathrm{i} \sin \left(\frac{2 \pi p_{2}}{n+2}\right)
$$

and the $T-Q$ relations, which now have the canonical form

$$
\tau(\mathrm{i} \lambda) \alpha_{ \pm}(\theta)=\alpha_{ \pm}(\theta+\mathrm{i} \pi)+\alpha_{ \pm}(\theta-\mathrm{i} \pi)
$$

(recall that $\tau(\mathrm{i} \lambda)=\tau(-\mathrm{i} \lambda)$ and $\lambda=\mathrm{e}^{\frac{\theta}{n}}$ ). 
Using the WKB approximation, one can explore the asymptotic behaviour of the connection coefficients for $\theta \rightarrow+\infty$. This leads to the asymptotic expansions similar to formulae (4.9) for $\zeta_{ \pm}(\theta)$. In particular, it is expected that for $\Re e(\theta) \rightarrow+\infty$ and $|\Im m(\theta)|<\frac{\pi}{2}(n+2)$,

$$
\alpha_{ \pm}(\theta) \asymp \mathrm{e}^{\mp \mathrm{i} \pi p_{2}} \tilde{\alpha}_{ \pm}\left(\mathrm{e}^{\frac{\mathrm{i} \pi}{2}} \mathrm{e}^{-\frac{\theta}{n+2}}\right) \quad \exp \left(-\frac{\pi}{\sin \left(\frac{\pi n}{2}\right)} \mathrm{e}^{\theta}\right)
$$

where $\tilde{\alpha}_{ \pm}(\tilde{\lambda})$ stand for the formal power series of the form

$$
\tilde{\alpha}_{ \pm}(\tilde{\lambda})=(\tilde{\lambda})^{ \pm 2 p_{2}} \exp \left(-\sum_{m=1}^{\infty} \tilde{\mathfrak{s}}_{m}^{( \pm)} \tilde{\lambda}^{2 m}\right)
$$

The coefficients $\left\{\tilde{\mathfrak{s}}_{m}^{( \pm)}\right\}_{m=1}^{\infty}$ are dual nonlocal IM, which are algebraically expressed through the set $\left\{\tilde{\mathfrak{t}}_{m}^{( \pm)}\right\}_{m=1}^{\infty}$ by means of the formal $T-Q$ relation (to be compared with eq. (4.69)):

$$
\tilde{\tau}(\tilde{\lambda}) \tilde{\alpha}_{ \pm}(\tilde{\lambda})=\tilde{\alpha}_{ \pm}(\tilde{q} \tilde{\lambda})+\tilde{\alpha}_{ \pm}\left(\tilde{q}^{-1} \tilde{\lambda}\right)
$$

where $\tilde{q}=\mathrm{e}^{-\frac{\mathrm{i} \pi}{n+2}}$. Substituting the formal power series (2.44) and (4.71) in (4.72), one can easily derive the relations between these two sets of dual nonlocal IM. For example

$$
\tilde{\mathfrak{s}}_{1}^{( \pm)}=\frac{\tilde{\mathfrak{t}}_{1}}{[1]\left[1 \pm 2 p_{2}\right]}, \quad \tilde{\mathfrak{s}}_{2}^{( \pm)}=\frac{\tilde{\mathfrak{t}}_{2}}{[2]\left[2 \pm 2 p_{2}\right]}-\frac{\tilde{\mathfrak{t}}_{1}^{2}}{[1][2]\left[1 \pm 2 p_{2}\right]\left[2 \pm 2 p_{2}\right]}+\frac{\tilde{\mathfrak{t}}_{1}^{2}}{2[1]^{2}\left[1 \pm 2 p_{2}\right]^{2}}
$$

where we use the shortcut notation $[x]=2 \sin \left(\frac{\pi x}{n+2}\right)$.

Unlike for $\alpha_{ \pm}$, the large- $\theta$ asymptotics of the operators $\beta_{ \pm}$include a contribution from the local IM; for $\Re e(\theta) \rightarrow+\infty$ and $|\Im m(\theta)|<\pi$, they read as

$$
\beta_{ \pm}(\theta) \asymp \tilde{\alpha}_{ \pm}\left(\mathrm{e}^{-\frac{\theta}{n+2}}\right) \exp \left(-2 \theta \mathrm{e}^{\theta}-\sum_{j=1}^{\infty} \mathfrak{i}_{2 j-1} \mathrm{e}^{-\theta(2 j-1)}\right) .
$$

Finally, using the formulae $(4.37),(4.58),(4.59),(4.65),(4.66)$, it is straightforward to show that the three sets of connection coefficients are not functionally independent, they satisfy the relations

$$
\begin{aligned}
& \zeta_{+}(\theta)=\frac{\mathrm{i} \mathrm{e}^{-\mathrm{i} \pi \mathrm{e}^{\theta}}}{2 \sin \left(\frac{2 \pi p_{2}}{n+2}\right)}\left[\mathrm{e}^{-\mathrm{i} \pi p_{2}} \beta_{-}(\theta) \alpha_{+}\left(\theta-\frac{\mathrm{i} \pi n}{2}\right)-\mathrm{e}^{+\mathrm{i} \pi p_{2}} \beta_{+}(\theta) \alpha_{-}\left(\theta-\frac{\mathrm{i} \pi n}{2}\right)\right] \\
& \zeta_{-}(\theta)=\frac{\mathrm{i} \mathrm{e}^{+\mathrm{i} \pi \mathrm{e}^{\theta}}}{2 \sin \left(\frac{2 \pi p_{2}}{n+2}\right)}\left[\mathrm{e}^{-\mathrm{i} \pi p_{2}} \beta_{-}(\theta+\mathrm{i} \pi) \alpha_{+}\left(\theta-\frac{\mathrm{i} \pi n}{2}\right)-\mathrm{e}^{+\mathrm{i} \pi p_{2}} \beta_{+}(\theta+\mathrm{i} \pi) \alpha_{-}\left(\theta-\frac{\mathrm{i} \pi n}{2}\right)\right] .
\end{aligned}
$$

It turns out these formulae and the quantum Wronskian relations, supplemented by the Hermiticity (4.12), (4.62), (4.67) and the analyticity of the operators $\alpha_{ \pm}(\theta), \beta_{ \pm}(\theta), \zeta_{ \pm}(\theta)$ constitute a very restrictive set of conditions. In particular, it leads to the important relation (see appendix $\mathrm{C}$ )

$$
\begin{aligned}
& \alpha_{ \pm}\left(\theta-\frac{\mathrm{i} \pi(n+1)}{2}\right) \alpha_{ \pm}\left(\theta+\frac{\mathrm{i} \pi(n+1)}{2}\right)-\alpha_{ \pm}\left(\theta-\frac{\mathrm{i} \pi(n-1)}{2}\right) \alpha_{ \pm}\left(\theta+\frac{\mathrm{i} \pi(n-1)}{2}\right) \\
& \quad=\beta_{ \pm}\left(\theta-\frac{\mathrm{i} \pi}{2}\right) \beta_{ \pm}\left(\theta+\frac{\mathrm{i} \pi}{2}\right)
\end{aligned}
$$




\subsection{NLIE for the vacuum eigenvalues}

The ODE/IQFT correspondence allows one, in principle, to find the spectrum of the commuting family of operators by numerically solving differential equations. Such a numerical procedure is especially convenient for the calculation of the eigenvalues of the operators $\beta_{ \pm}(\theta)$. However, it is a highly non-trivial task to, say, extract the eigenvalues of the chiral transfer-matrix $\tau(\lambda)$ directly from the differential equations. Here we demonstrate how the functional relations and analytic conditions for the connection coefficients can be used to derive a system of Non-Linear Integral Equations (NLIE) which prove to be highly efficient in numerical work [61-63]. We will mostly focus on the vacuum eigenvalues.

For our purposes it is useful to rewrite eq. (4.76) using the notation

$$
\mathrm{e}^{\mathrm{i} \varepsilon(\theta)}=\exp \left(2 \pi \mathrm{i}\left(\mathrm{e}^{\theta}-p_{2}\right)\right) \frac{\alpha_{+}\left(\theta-\frac{\mathrm{i} \pi n}{2}\right)}{\alpha_{+}\left(\theta+\frac{\mathrm{i} \pi n}{2}\right)} .
$$

Focusing on the case where the subscript in (4.76) is "+", one has

$$
1-\mathrm{e}^{4 \pi \mathrm{e}^{\theta}+\mathrm{i} \varepsilon\left(\theta+\frac{\mathrm{i} \pi}{2}\right)-\mathrm{i} \varepsilon\left(\theta-\frac{\mathrm{i} \pi}{2}\right)}=\frac{\beta_{+}\left(\theta-\frac{\mathrm{i} \pi}{2}\right) \beta_{+}\left(\theta+\frac{\mathrm{i} \pi}{2}\right)}{\alpha_{+}\left(\theta-\frac{\mathrm{i} \pi(n+1)}{2}\right) \alpha_{+}\left(\theta+\frac{\mathrm{i} \pi(n+1)}{2}\right)} .
$$

In order to define the operator $\varepsilon(\theta)$ itself, one should specify the branch of the logarithm $\frac{1}{\mathrm{i}} \log \left(\mathrm{e}^{\mathrm{i} \varepsilon(\theta)}\right)$. This can be done by supplementing (4.77) with the leading asymptotic behaviour

$$
\varepsilon(\theta) \rightarrow 4 \pi \mathrm{e}^{\theta}-\frac{4 \pi p_{2}}{n+2}+o(1) \quad \text { as } \quad \theta \rightarrow+\infty,
$$

which is chosen to be consistent with the asymptotic formula (4.70). As follows from eq. (4.67), the operator $\varepsilon(\theta)$, thus defined and acting in the Fork space $\mathcal{F}_{p_{1}, p_{2}}$, satisfies the Hermiticity condition

$$
[\varepsilon(\theta)]^{\dagger}=\varepsilon\left(\theta^{*}\right)
$$

Let us emphasize that (4.78)-(4.80) are operator relations for the commuting family, and therefore the same relations hold true for the eigenvalues corresponding to any common eigenvector $|\psi\rangle$ in the Fock space. Another important general property (which justifies the "strange" first factor in the definition (4.77)) concerns the zeroes of the eigenvalue $\beta_{+}^{(\psi)}(\theta)$. Namely it is easy to show that (see appendix C)

$$
\text { if } \theta_{j}: \beta_{+}^{(\psi)}\left(\theta_{j}\right)=0, \text { then } \exp \left(\mathrm{i} \varepsilon^{(\psi)}\left(\theta_{j}-\mathrm{i} \pi\right)\right)=-1 \text {. }
$$

Notice that since $\beta_{+}^{(\psi)}(\theta)$ is a real analytic and periodic function in $\theta$ with period $2 \pi \mathrm{i}$, it is sufficient to consider its zeroes in the strip $0 \leq \Im m(\theta) \leq \pi$, only.

In the case of the vacuum eigenvalues, our numerical work suggests that for $\frac{2 p_{2}}{n+2}>-\frac{1}{2}$ all the zeroes of $\beta_{+}^{(\mathrm{vac})}(\theta)$ in the strip $|\Im m(\theta)| \leq \pi$ are simple, located on the boundary $\Im m(\theta)=\pi$, accumulate toward $\Re e(\theta) \rightarrow+\infty$ and satisfy the condition

$$
\varepsilon^{(\mathrm{vac})}\left(\theta_{j}-\mathrm{i} \pi\right)=\pi(2 j-1) \quad(j=1,2, \ldots) .
$$


This "quantization condition" supplemented by the asymptotic formula (4.70), leads to an equation determining the vacuum roots of $\beta_{+}^{(\mathrm{vac})}(\theta)$ which is asymptotically exact as $j \rightarrow+\infty$ :

$$
\exp \left(\theta_{j}^{(\mathrm{vac})}\right) \equiv-\rho_{j}: \quad \rho_{j} \asymp \frac{1}{2}\left(j-\frac{1}{2}+\frac{2 p_{2}}{n+2}\right)-\frac{1}{2 \pi} \sum_{m=1}^{\infty} \tilde{s}_{m}\left(p_{1}, p_{2}\right) \sin \left(\frac{2 \pi m}{n+2}\right)\left(\rho_{j}\right)^{-\frac{2 m}{n+2}}
$$

where $\tilde{s}_{m}\left(p_{1}, p_{2}\right)$ stands for the vacuum eigenvalues of the dual nonlocal IM $\tilde{\mathfrak{s}}_{m}^{+}$ in (4.71), (4.73). In particular

$$
\tilde{s}_{1}\left(p_{1}, p_{2}\right)=-\left(\frac{n+2}{2}\right)^{\frac{2}{n+2}} \frac{\Gamma\left(\frac{1}{n+2}\right) \Gamma\left(\frac{1}{2}-\frac{1}{n+2}\right)}{4 \sqrt{\pi}}\left[\frac{n}{n+4}-\frac{4 p_{1}^{2}}{1-4 p_{2}^{2}}\right] \frac{\Gamma\left(1+\frac{2 p_{2}+1}{n+2}\right)}{\Gamma\left(\frac{2 p_{2}-1}{n+2}\right)} .
$$

Additional analytical input required for the derivation of the NLIE, is the behaviour at $\Re e(\theta) \rightarrow-\infty$. It can be studied along the following line: using the formulae (4.75) and the quantum Wronskian relations one can express $\alpha_{+}^{(\mathrm{vac})}$ in terms of $\zeta_{ \pm}^{(\mathrm{vac})}$ and $\beta_{+}^{(\mathrm{vac})}$. The asymptotics of $\zeta_{ \pm}^{(\mathrm{vac})}(\theta)$ at $\theta \rightarrow-\infty$ are given by eqs. (4.30). The general structure of the $\theta \rightarrow-\infty$ behaviour of $\beta_{+}^{(\mathrm{vac})}$ is dictated by the operator valued series expansion (4.61). Using the differential equation (4.53), it is not difficult to find the following explicit expressions for the vacuum eigenvalues of the first expansion coefficients:

$$
b_{ \pm}^{(\mathrm{vac})}\left(p_{1}, p_{2}\right)=(n+2)^{-\frac{1}{2} \mp \frac{2 p_{2}}{n+2}} \frac{2 \pi \Gamma\left(1 \pm 2 p_{2}\right)}{\Gamma\left(\frac{1}{2}-\mathrm{i} p_{1} \pm p_{2}\right) \Gamma\left(\frac{1}{2}+\mathrm{i} p_{1} \pm p_{2}\right) \Gamma\left(1 \pm \frac{2 p_{2}}{n+2}\right)}
$$

and (to be compared with the coefficient $f_{1,0}$ in eq. (4.33))

$$
b_{1}^{( \pm, \mathrm{vac})}\left(p_{1}, p_{2}\right)=-\psi\left(\frac{1}{2}-\mathrm{i} p_{1} \pm p_{2}\right)-\psi\left(\frac{1}{2}+\mathrm{i} p_{1} \pm p_{2}\right)+\psi\left(1+\frac{n}{2}\right)+\gamma_{E}+2 \log 2-2 .
$$

Now it is straightforward to derive the leading asymptotic behaviour of the vacuum eigenvalue of the operator $\varepsilon(\theta)$ (4.77), (4.79). Below we list explicit formulae for $\varepsilon^{(\mathrm{vac})}(\theta)$ and for the vacuum eigenvalues of the operator

$$
\omega(\theta) \equiv \log \left(\mathrm{e}^{\mathrm{i} \varepsilon\left(\theta-\frac{\mathrm{i} \pi}{2}\right)-\mathrm{i} \varepsilon\left(\theta+\frac{\mathrm{i} \pi}{2}\right)-4 \pi \mathrm{e}^{\theta}}-1\right), \quad \omega(\theta) \rightarrow 4 \pi \mathrm{e}^{\theta}+o(1) \quad \text { as } \quad \theta \rightarrow+\infty .
$$

(a) For real $p_{1} \neq 0$ and $p_{2}>-\frac{1}{2}$ :

$$
\begin{aligned}
\varepsilon^{(\mathrm{vac})}(\theta) & =-2 \pi p_{2}+\frac{1}{\mathrm{i}} \log \left[\frac{\sin \left(\frac{2 p_{1}}{n}\left(\theta_{0}-\theta+\frac{\mathrm{i} \pi n}{2}\right)\right)}{\sin \left(\frac{2 p_{1}}{n}\left(\theta_{0}-\theta-\frac{\mathrm{i} \pi n}{2}\right)\right)}\right]+o\left(\theta^{-\infty}\right) \\
\exp \left(-\omega^{(\mathrm{vac})}(\theta)\right) & =\frac{\sinh ^{2}\left(\frac{\pi(n-1) p_{1}}{n}\right)}{\sinh \left(2 \pi p_{1}\right) \sinh \left(\frac{2 \pi p_{1}}{n}\right)}+\frac{\sin ^{2}\left(\frac{2 p_{1}}{n}\left(\theta_{0}-\theta\right)\right)}{\sinh \left(2 \pi p_{1}\right) \sinh \left(\frac{2 \pi p_{1}}{n}\right)}+o\left(\theta^{-\infty}\right)
\end{aligned}
$$

where

$$
\theta_{0}=\log (n)+\frac{n}{4 \mathrm{i} p_{1}} \log \left[\frac{\Gamma\left(1+2 \mathrm{i} p_{1}\right) \Gamma\left(1+\frac{2 \mathrm{i} p_{1}}{n}\right)}{\Gamma\left(1-2 \mathrm{i} p_{1}\right) \Gamma\left(1-\frac{2 \mathrm{i} p_{1}}{n}\right)} \frac{\Gamma^{2}\left(\frac{1}{2}+p_{2}-\mathrm{i} p_{1}\right)}{\Gamma^{2}\left(\frac{1}{2}+p_{2}+\mathrm{i} p_{1}\right)}\right] .
$$


(b) For $p_{1}=0$ and $p_{2}>-\frac{1}{2}$ :

$$
\begin{aligned}
\varepsilon^{(\mathrm{vac})}(\theta) & =-2 \pi p_{2}+\frac{1}{\mathrm{i}} \log \left[\frac{\theta_{0}-\theta+\frac{\mathrm{i} \pi n}{2}}{\theta_{0}-\theta-\frac{\mathrm{i} \pi n}{2}}\right]+o\left(\theta^{-\infty}\right) \\
\exp \left(-\omega^{(\mathrm{vac})}(\theta)\right) & =\frac{\left(\theta_{0}-\theta\right)^{2}}{n \pi^{2}}+\frac{1}{4 n}(n-1)^{2}+o\left(\theta^{-\infty}\right),
\end{aligned}
$$

where

$$
\theta_{0}=\log (n)-(1+n) \gamma_{E}-n \psi\left(\frac{1}{2}+p_{2}\right)
$$

(c) For pure imaginary $p_{1} \equiv \frac{\mathrm{i}}{2} \mathfrak{m}$ with $0<\mathfrak{m}<\frac{n}{2}$ and real $p_{2} \equiv \mathfrak{j}+\frac{1}{2}$ such that $\mathfrak{j}-\frac{1}{2} \mathfrak{m}>-1$ :

$$
\begin{aligned}
& \varepsilon^{(\operatorname{vac})}(\theta)=-\pi(2 \mathfrak{j}+1-\mathfrak{m})+\frac{2 \pi \mathfrak{m} n^{-\frac{2 \mathfrak{m}}{n}}}{\Gamma^{2}(1+\mathfrak{m})} \frac{\Gamma\left(1-\frac{\mathfrak{m}}{n}\right)}{\Gamma\left(1+\frac{\mathfrak{m}}{n}\right)} \frac{\Gamma^{2}\left(1+\mathfrak{j}+\frac{\mathfrak{m}}{2}\right)}{\Gamma^{2}\left(1+\mathfrak{j}-\frac{\mathfrak{m}}{2}\right)} \mathrm{e}^{\frac{2 \mathfrak{m} \theta}{n}}+o\left(\mathrm{e}^{\frac{2 \mathfrak{m} \theta}{n}}\right) \\
& \omega^{(\operatorname{vac})}(\theta)=\frac{2 \mathfrak{m}}{n} \theta-2 \log \left[n^{\frac{1}{2}+\frac{\mathfrak{m}}{n}} \frac{\Gamma\left(1+\mathfrak{j}-\frac{\mathfrak{m}}{2}\right) \Gamma(\mathfrak{m}) \Gamma\left(1+\frac{\mathfrak{m}}{n}\right)}{2 \pi \Gamma\left(1+\mathfrak{j}+\frac{\mathfrak{m}}{2}\right)}\right]+O\left(\mathrm{e}^{\frac{2 \theta}{n}}, \mathrm{e}^{\theta}\right)
\end{aligned}
$$

Notice that eqs. (4.89) with $p_{2}=\mathfrak{j}+\frac{1}{2}$ and

$$
\mathfrak{j}=\frac{1}{2}, 1, \ldots, \frac{1}{2}\left[\frac{n}{2}\right] \quad(n=2,3,4, \ldots)
$$

can be applied to the parafermion case with $\mathfrak{m}=0$. For positive integer $\mathfrak{m}$, restricted to

$$
1 \leq \mathfrak{m} \leq\left[\frac{n-1}{2}\right], \quad \mathfrak{j}-\frac{1}{2} \mathfrak{m} \geq 0,
$$

eqs. (4.90) should be used.

It is expected that for the cases (b) and (c), the function $\alpha^{(\mathrm{vac})}(\theta)$ does not have any zeroes in the strip $|\Im m(\theta)|<\frac{\pi}{2}(n+2)$. However, as it follows from the asymptotic behaviour (4.88) and formula (4.77), for $p_{1} \neq 0$ and $p_{2}>-\frac{1}{2}$ it has a sequence of zeroes, $\left\{\theta_{m}^{(\alpha)}\right\}_{m=1}^{\infty}$, extending towards $-\infty$ along the real axis such that

$$
\theta_{m}^{(\alpha)}=\theta_{0}-\frac{\pi n}{2 p_{1}} m+o\left(\left(m / p_{1}\right)^{-\infty}\right) \quad(m=1,2, \ldots) .
$$

Again, we expect that there are no other zeroes apart from $\left\{\theta_{m}^{(\alpha)}\right\}_{m=1}^{\infty}$ in the strip $|\Im m(\theta)|<$ $\frac{\pi}{2}(n+2)$. In principle, these properties should be rigorously derived from the differential equation (4.53). Unfortunately, we don't have a proof at this moment and we formulate the statements as a conjecture. Notice that the domain of applicability of the large- $\theta$ asymptotic expansion (4.70) is also restricted to the same strip.

The outlined analytic properties fully determine all of the vacuum eigenvalues of the commuting operators acting in the Fock space $\mathcal{F}_{p_{1}, p_{2}}$. Practically, they can be used to derive a closed system of integral equations which involve the vacuum eigenvalues of $\varepsilon^{(\mathrm{vac})}(\theta)$ and $\omega^{(\mathrm{vac})}(\theta)$. A few useful formulae appearing in the intermediate steps of the derivation are 
given in appendices $\mathrm{C}$ and $\mathrm{D}$. With the parameters $p_{1}$ and $p_{2}$ as in (b) and ((c)) above, the final result reads as follows:

$$
\begin{aligned}
\varepsilon(\theta-\mathrm{i} \gamma)= & 4 \pi \mathrm{e}^{\theta-\mathrm{i} \gamma}-2 \pi k+\int_{-\infty}^{\infty} \frac{\mathrm{d} \theta^{\prime}}{2 \pi \mathrm{i}}\left[G\left(\theta-\theta^{\prime}-2 \mathrm{i} \gamma\right)\left(L\left(\theta^{\prime}-\mathrm{i} \gamma\right)\right)^{*}\right. \\
& \left.-G\left(\theta-\theta^{\prime}\right) L\left(\theta^{\prime}-\mathrm{i} \gamma\right)\right]+\int_{-\infty}^{\infty} \frac{\mathrm{d} \theta^{\prime}}{2 \pi} G_{1}\left(\theta-\theta^{\prime}-\mathrm{i} \gamma\right) \log \left(1+\mathrm{e}^{-\omega\left(\theta^{\prime}\right)}\right) \\
\omega(\theta)= & 4 \pi \mathrm{e}^{\theta}+\Im m\left[\int_{-\infty}^{\infty} \frac{\mathrm{d} \theta^{\prime}}{\pi} G_{1}\left(\theta-\theta^{\prime}+\mathrm{i} \gamma\right) L\left(\theta^{\prime}-\mathrm{i} \gamma\right)\right] \\
& -\int_{-\infty}^{\infty} \frac{\mathrm{d} \theta^{\prime}}{\pi} G_{2}\left(\theta-\theta^{\prime}\right) \log \left(1+\mathrm{e}^{-\omega\left(\theta^{\prime}\right)}\right) \\
L(\theta)= & \log \left(1+\mathrm{e}^{-\mathrm{i} \varepsilon(\theta)}\right) .
\end{aligned}
$$

Here we drop the superscript "(vac)" in the notation for the vacuum eigenvalues, and the star "*" as usual, stands for complex conjugation. The constant $\gamma$ is an arbitrary number belonging to the open segment $0<\gamma<\frac{\pi}{2}$. We also swap $p_{2}$ for

$$
k=\frac{2 p_{2}}{n+2},
$$

and the kernels read explicitly as

$$
\begin{aligned}
G(\theta) & =\frac{\sin \left(\frac{2 \pi}{n+2}\right)}{(n+2) \sinh \left(\frac{\theta+\mathrm{i} \pi}{n+2}\right) \sinh \left(\frac{\theta-\mathrm{i} \pi}{n+2}\right)} \\
G_{1}(\theta) & =\frac{\sin \left(\frac{2 \pi}{n+2}\right) \sin \left(\frac{\pi}{n+2}\right) \sinh \left(\frac{2 \theta}{n+2}\right)}{(n+2) \sinh \left(\frac{\theta+\frac{\mathrm{i} \pi}{2}}{n+2}\right) \sinh \left(\frac{\theta-\frac{\mathrm{i} \pi}{2}}{n+2}\right) \sinh \left(\frac{\theta+\frac{3 \mathrm{i} \pi}{2}}{n+2}\right) \sinh \left(\frac{\theta-\frac{3 \mathrm{i} \pi}{2}}{n+2}\right)} \\
G_{2}(\theta) & =G(\theta)-\frac{\sin \left(\frac{4 \pi}{n+2}\right)}{2(n+2) \sinh \left(\frac{\theta+2 \mathrm{i} \pi}{n+2}\right) \sinh \left(\frac{\theta-2 \mathrm{i} \pi}{n+2}\right)} .
\end{aligned}
$$

It is important to keep in mind that the integral equations should be supplemented by the asymptotics for the vacuum eigenvalues given by eqs. (4.89)-(4.90). For real $p_{1} \neq 0$ and $p_{2}>-\frac{1}{2}$ (as in case (a) above) the integral equations must be modified by adding extra source terms to the r.h.s. The corresponding NLIE is given by formulae (D.1), (D.2) in appendix D along with some explanations.

Once the system of NLIE is solved, the numerical data can be used to reconstruct the vacuum eigenvalues of $\alpha^{(\mathrm{vac})}(\theta), \beta^{(\mathrm{vac})}(\theta)$ and $\tau^{(\mathrm{vac})}(\mathrm{i} \lambda)$. The corresponding formulae are given by (C.6)-(C.7) from appendix C. Expressions for the vacuum eigenvalues of the local and dual nonlocal integrals of motion are present there as well. In figure 6 numerical results for $\tau^{\text {(vac) }}$ in the parafermionic vacuum with $n=3$ and $2 \mathfrak{j}=\mathfrak{m}=1\left(p_{1}=\frac{i}{2}, p_{2}=1\right)$ are shown alongside the results from the Bethe ansatz. Also, for numerous cases, we compared the numerical results for the connection coefficients computed from the NLIE with those obtained by direct integration of the ordinary differential equations (4.53). The agreement we found in all cases justifies the assumptions made within the derivation of the NLIE system. 


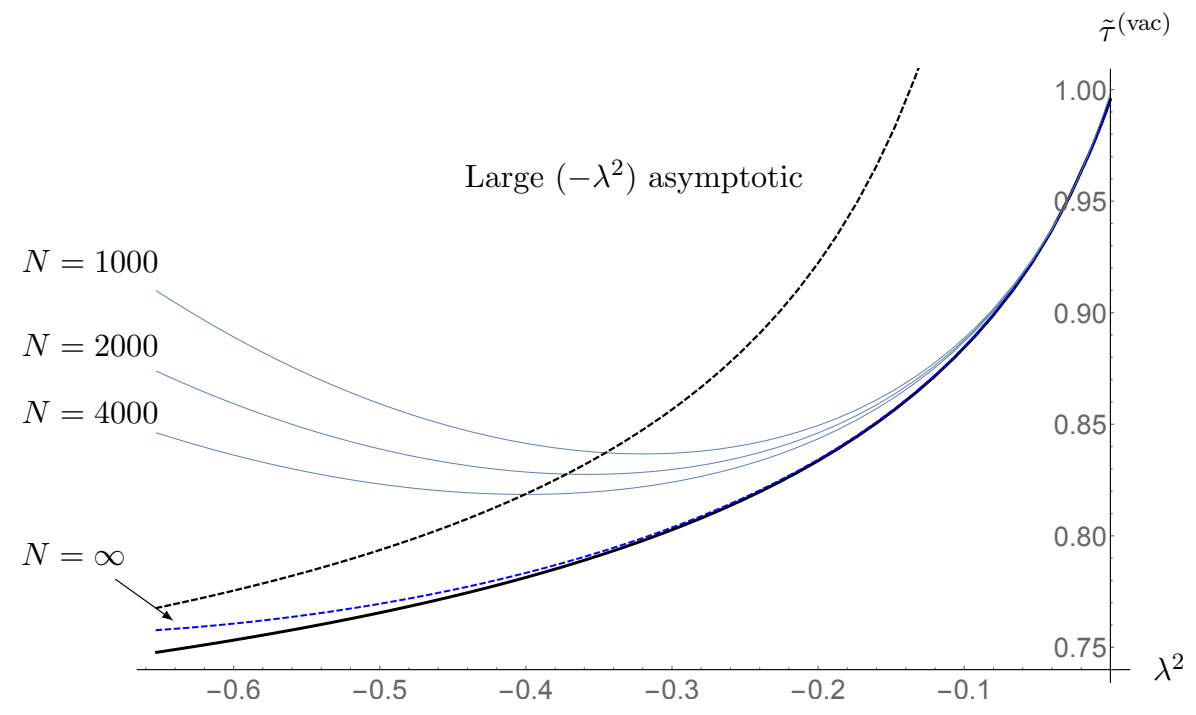

Figure 6. A plot of the scaling function $\tilde{\tau}^{(\text {vac })}=\tau^{(\mathrm{vac})} \times \exp \left(2 \pi\left(-\lambda^{2}\right)^{\frac{3}{2}}\right)$ for the parafermion vacuum with $n=3$, and $2 \mathfrak{j}=\mathfrak{m}=1$. The thick black line comes from the numerical solution of the NLIE system (4.94). The blue curves are the same as in the plot appearing in the right panel of figure 9 from appendix A. They were obtained from the numerical solution of the Bethe ansatz equations for finite $N$ and subsequently interpolating the data to $N=\infty$. The large $\left(-\lambda^{2}\right)$ asymptotic is given by eqs. (2.43), (2.44).

\section{Integrable structures in the sausage}

We now turn to the main subject of interest - the sausage model. First of all, we recall some basic facts concerning this quantum field theory. For more details, see, e.g., [44].

\subsection{Basic facts about the quantum sausage}

At the beginning of section 2, we briefly touched on the one loop renormalization in the sausage model. Let us introduce the renormalized coupling $\kappa_{r}$ which substitutes the bare coupling $\kappa(2.2)$ :

$$
\frac{1-\kappa_{r}}{1+\kappa_{r}}=\left(E_{*} / E\right)^{\frac{2}{n}} .
$$

Here $E$ stands for a typical energy scale, which, in the case under consideration, can be identified with the inverse of the circumference of the space-time cylinder, $R^{-1}$. Recall also that $n \equiv \frac{2 \pi}{\hbar}$ and $E_{*}$ is a RG invariant energy scale appearing in the theory through the mechanism of dimensional transmutation. Within the one-loop approximation the bare coupling is replaced by $\kappa_{r}$, so that the renormalized sausage metric is given by

$$
G_{a b}^{(\mathrm{ren})} \mathrm{d} X^{a} \mathrm{~d} X^{b}=\frac{n}{2 \pi} \frac{(\mathrm{d} \phi)^{2}+(\mathrm{d} \alpha)^{2}}{\frac{1}{2}\left(\kappa_{r}^{-1}+\kappa_{r}\right)+\frac{1}{2}\left(\kappa_{r}^{-1}-\kappa_{r}\right) \cosh (2 \phi)},
$$

where we use the pair of real coordinates $X^{a}=(\phi, \alpha)(1.17)$. Consider the ultraviolet regime where $E \gg E^{*}$, i.e., $1-\kappa_{r} \ll 1$. In this case, the central region of the sausage 
depicted in figure 2 looks like a long cylinder equipped with the flat metric. If one formally sets $\kappa_{r}=1$ in (5.2), ignoring the presence of the two infinitely separated tips, then

$$
G_{a b}^{(\mathrm{ren})} \mathrm{d} X^{a} \mathrm{~d} X^{b} \approx \frac{n}{2 \pi}\left((\mathrm{d} \phi)^{2}+(\mathrm{d} \alpha)^{2}\right) .
$$

The NLSM action corresponding to this metric has the form of the massless Gaussian $\operatorname{model}^{13}$

$$
\mathcal{A}_{0}=\frac{n}{4 \pi} \int \mathrm{d}^{2} x\left(\left(\partial_{\mu} \phi\right)^{2}+\left(\partial_{\mu} \alpha\right)^{2}\right)
$$

We now apply the $T$-duality transformation to the field $\alpha$, i.e., we replace $\alpha$ by the $T$ dual field $\vartheta$ such that $\partial_{\mu} \alpha=\frac{1}{\sqrt{n+2}} \epsilon_{\mu \nu} \partial_{\nu} \vartheta$ (the difference between $n$ and $n+2$ is ignored here, since $n$ is assumed to be large). The substitution of $(\phi, \alpha)$ by the pair $(\varphi, \vartheta)$, with $\phi=\frac{1}{\sqrt{n}} \varphi$, brings the action to the form

$$
\tilde{\mathcal{A}}_{0}=\frac{1}{4 \pi} \int \mathrm{d}^{2} x\left(\left(\partial_{\mu} \varphi\right)^{2}+\left(\partial_{\mu} \vartheta\right)^{2}\right)
$$

The advantage of $\tilde{\mathcal{A}}_{0}$ compared to the action (5.4), is that it allows one to easily incorporate the effects of the sausage tips. The central region with the left tip of the sausage form the cigar, and the corresponding NLSM, as it was mentioned in section 2.3, admits the dual description in terms of the sine-Liouville action

$$
\tilde{\mathcal{A}}^{(\text {left })}=\tilde{\mathcal{A}}_{0}+2 \mathcal{M} \int \mathrm{d}^{2} x \mathrm{e}^{-\sqrt{n} \varphi} \cos (\sqrt{n+2} \vartheta) .
$$

Clearly, the cigar NLSM whose target space is glued from the right tip and the central region, is governed by the action which is related to (5.6) by the flip $\varphi \mapsto-\varphi$, i.e.,

$$
\tilde{\mathcal{A}}^{\text {(right) }}=\tilde{\mathcal{A}}_{0}+2 \mathcal{M} \int \mathrm{d}^{2} x \mathrm{e}^{+\sqrt{n} \varphi} \cos (\sqrt{n+2} \vartheta) .
$$

At this point one can guess that the sausage NLSM admits the dual description by means of the renormalized action

$$
\tilde{\mathcal{A}}^{\text {(saus) }}=\int \mathrm{d}^{2} x\left(\frac{1}{4 \pi}\left(\left(\partial_{\mu} \varphi\right)^{2}+\left(\partial_{\mu} \vartheta\right)^{2}\right)+4 \mathcal{M} \cosh (\sqrt{n} \varphi) \cos (\sqrt{n+2} \vartheta)\right) .
$$

Remarkably, the naive guess turns out to be correct! The dual form of the sausage model was originally proposed by Aleosha Zamolodchikov and there are many arguments to support its validity, a few of which will be mentioned later in the text. Contrary to the sine-Liouville model where the dimensionless $\mathcal{M}$ is a somewhat fake parameter, the coupling $\mathcal{M}$ in (5.8) is an important, dimensionful characteristic of the theory. Notice that the field $\cosh (\sqrt{n} \varphi) \cos (\sqrt{n+2} \vartheta)$ has the scale dimensions equal to one w.r.t. the conventional energy momentum tensor of the "unperturbed" free theory (5.5). Therefore $\mathcal{M}$ has dimensions of energy, i.e., $\mathcal{M} \propto E_{*}$ up to some dimensionless constant.

Consider now the general structure of the Hilbert space of the sausage NLSM in finite volume equipped with the boundary conditions of the form (2.69). The quantum number

\footnotetext{
${ }^{13}$ In what follows the Planck constant $\hbar=\frac{2 \pi}{n}$ will be always included in the action.
} 
$m$ in that formula must take integer values only, it is a conserved charge associated with the $\mathrm{U}(1)$-isometry of the sausage metric. In what follows we will focus on the neutral sector of the theory with $m=0$, and therefore

$$
\varphi(t, x+R)=\varphi(t, x), \quad \vartheta(t, x+R)=\vartheta(t, x) .
$$

Since the action (5.8) and the boundary conditions are both invariant under the transformation $\vartheta \mapsto \vartheta+\frac{2 \pi}{\sqrt{n+2}}$, the space of the neutral states of the sausage model is somewhat similar to the Hilbert space of a quantum particle in a periodic potential: it is split on the orthogonal subspaces $\mathcal{H}_{k}^{(K)}$ characterized by the quasimomentum restricted to the first Brillouin zone, $-\frac{1}{2}<k<\frac{1}{2}$, and a positive integer $K$ - the band number. Our considerations below will be mostly restricted to the $k$-vacuum, $|v a c\rangle_{k} \in \mathcal{H}_{k}^{(1)}$ - the lowest energy neutral state in the first band.

In section 2.3 it was mentioned that the Hilbert space of the cigar/sine-Liouville theory is classified w.r.t. the action of a certain $\mathfrak{W} \otimes \overline{\mathfrak{W}}$-algebra. The $W$-algebras related by the reflection $\varphi \rightarrow-\varphi$ are algebraically isomorphic (for details see, e.g., [42, 44]). This property allows one to identify the spaces of states for the "left" and "right" sine-Liouville models (5.6) and (5.7) which can be then interpreted as an extended Hilbert space of the sausage NLSM. However, contrary to the case of the cigar NLSM, instead of the continuous summation as in (2.60), the zero-mode momentum $p_{1}$ in the sausage takes a certain discrete set of admissible values which depends on $R$. As $\mathcal{M} R \rightarrow 0$ the mechanism of the quantization of $p_{1}$ is similar to that in the sinh-Gordon model considered in ref. [64]. The discussions from this work can be easily adopted to our problem (see, e.g., [42]).

When $\mathcal{M} R \ll 1$, the quantization condition which determines the value of $p_{1}=p_{1}(R)$ for the $k$-vacuum, $|v a c\rangle_{k} \in \mathcal{H}_{k}^{(1)}$, reads as follows

$$
-\frac{8 p_{1}}{n} \log \left(\frac{\mathcal{M} R}{2}\right)+2 \delta\left(p_{1}, p_{2}\right)=2 \pi .
$$

Here $p_{2} \equiv \frac{1}{2}(n+2) k, \delta\left(p_{1}, p_{2}\right)=-\mathrm{i} \log S_{0}(\mathbf{p})$ and $S_{0}(\mathbf{p})$ is the overall scalar factor for the $S$-matrix (2.66). This factor was first derived by A. and Al. Zamolodchikov [47] and, using the notation of $B_{p_{1}, p_{2}}$ from (4.31), it is given by $S_{0}\left(p_{1}, p_{2}\right)=-B_{-p_{1}, p_{2}} / B_{p_{1}, p_{2}}$. Thus,

$$
\delta\left(p_{1}, p_{2}\right)=\frac{4 p_{1}}{n} \log (n)-\mathrm{i} \log \left[\frac{\Gamma\left(\frac{1}{2}-p_{2}-\mathrm{i} p_{1}\right) \Gamma\left(\frac{1}{2}+p_{2}-\mathrm{i} p_{1}\right)}{\Gamma\left(\frac{1}{2}-p_{2}+\mathrm{i} p_{1}\right) \Gamma\left(\frac{1}{2}+p_{2}+\mathrm{i} p_{1}\right)} \frac{\Gamma\left(1+2 \mathrm{i} p_{1}\right)}{\Gamma\left(1-2 \mathrm{i} p_{1}\right)} \frac{\Gamma\left(1+\frac{2 \mathrm{i} p_{1}}{n}\right)}{\Gamma\left(1-\frac{2 \mathrm{i} p_{1}}{n}\right)}\right]
$$

where the branch of the logarithm is chosen so that $\delta\left(0, p_{2}\right)=0$. The vacuum energy in the sector $\mathcal{H}_{k}^{(1)}$ is approximately the corresponding vacuum energy of the free theory (5.5), with the zero mode momentum for the field $\varphi$ determined by the quantization condition (5.10):

$$
E_{k}^{(\mathrm{vac})} \approx \frac{\pi}{R}\left(-\frac{1}{3}+\frac{4}{n}\left(p_{1}(R)\right)^{2}+(n+2) k^{2}\right) .
$$

This result can be obtained both from the theory described by the action (5.8) and directly from the sausage NLSM within the so-called minisuperspace approximation, which, in fact, is a strong argument for the validity of the dual description of the quantum sausage. 
A few comments to the formula (5.12) are in order. First of all a brief inspection of the scattering phase $\delta\left(p_{1}, p_{2}\right)$ (5.11), shows that the quantization condition can be applied literally only for $\left|p_{2}\right|<\frac{1}{2}$, or, equivalently, for $|k|<\frac{1}{n+2}$. For $p_{2}=\frac{1}{2}$, notice that eq. (5.10) has an $R$-independent solution $p_{1}=0$, whereas for $\frac{1}{n+2}<|k|<\frac{1}{2}$, the precise form of the quantization condition, to the best of our knowledge, is currently not known. The next comment deals with corrections to the approximate formula (5.12). A superficial analysis shows that for $n>2$ the main correction is of order $R^{\frac{4}{n}-1}$, while for $0<n<2$, a term $\propto R \log (R)$ dominates. The latter can be understood as the one-instanton contribution and has the following explicit form

$$
\delta E^{(1-\mathrm{inst})}=-\pi \mathcal{M}^{2} R\left(4 \log \left(R \Lambda^{(\mathrm{inst})}\right)+e(k)+O\left(p_{1}^{2}(R)\right)\right) .
$$

Here $\Lambda^{\text {(inst) }}$ is a cut-off energy scale which regularizes the contribution of the small-size instantons $[14,65-67]$ and

$$
e(k)=-2-2 \psi\left(\frac{1}{2}-\frac{(n+2) k}{2}\right)-2 \psi\left(\frac{1}{2}+\frac{(n+2) k}{2}\right) .
$$

Now, let us turn to the large- $R$ limit. In this limit, $E_{k}^{(\mathrm{vac})}$ contains an extensive part which is proportional to the spatial size of the system and does not depend on $k$. As follows from the results of ref. [14], the specific bulk energy $\mathcal{E} \equiv \lim _{R \rightarrow \infty} E_{k}^{(\mathrm{vac})} / R$ is given by

$$
\mathcal{E}=\pi \mathcal{M}^{2}\left(4 \log \left(\mathcal{M} / \Lambda^{(\mathrm{inst})}\right)+\pi \cot \left(\frac{\pi n}{2}\right)+2 \gamma_{E}+2 \psi\left(1+\frac{n}{2}\right)\right) .
$$

The large- $R$ behaviour of the difference $E_{k}^{(\mathrm{vac})}-R \mathcal{E}$ is dictated by the factorized scattering theory associated with the model. In the original work [14], it was proposed that the spectrum of the sausage model in infinite volume consists of a triplet of particles of the same mass $m$. The mass scale is simply related to the dimensionful coupling in the renormalized action (5.8):

$$
m=4 \pi \mathcal{M} .
$$

Two of the particles $A_{+}$and $A_{-}$carry the U(1) charge +1 and -1 , respectively, whereas the third particle $A_{0}$ is neutral. The factorized scattering is completely determined by the two-particle $S$-matrix which can be interpreted as a structure constant in the formal Zamolodchikov-Faddeev associative algebra:

$$
A_{a}\left(\theta_{1}\right) A_{b}\left(\theta_{2}\right)=S_{a b}^{c d}\left(\theta_{1}-\theta_{2}\right) A_{d}\left(\theta_{2}\right) A_{c}\left(\theta_{1}\right) .
$$

For convenience, we collect in appendix E explicit expressions for the scattering amplitude $S_{a b}^{c d}$ proposed in [14]. Taking a closer look at these amplitudes, one can observe that they are trivialized for $n=0$. This is consistent with the fact that the dual theory (5.8) can be understood, by use of the Coleman-Mandelstam bosonization, as an interacting theory of a massive scalar and Dirac fermion. At $n=0$, the interaction disappears and we end up with a theory of three non-interacting particles. In fact, this was the starting point of Al. Zamolodchikov's proposal for the dual description of the sausage. He performed 
perturbative calculations for small $n$ and found that the perturbative amplitudes match the small- $n$ expansion of the exact two-particle $S$-matrix.

As usual for a massive quantum field theory, the large- $R$ expansion of $E_{k}^{(\mathrm{vac})}-R \mathcal{E}$ can be represented in the form

$$
E_{k}^{(\mathrm{vac})}-R \mathcal{E}=\Delta E^{1-\text { particle }}+\Delta E^{2-\text { particle }}+\ldots,
$$

where the individual terms correspond to the virtual contributions of $N$-particle states. The one-particle contribution does not depend on the details of the interaction - it is the same as for the non-interacting theory and hence is given by

$$
\Delta E^{1-\text { particle }}=-\operatorname{Tr}_{1}\left(\boldsymbol{K}^{(1)}\right) \int_{-\infty}^{\infty} \frac{\mathrm{d} \theta}{2 \pi} m \cosh (\theta) \mathrm{e}^{-m R \cosh (\theta)} .
$$

Here the trace is taken over the isotopic component of the one-particle sector of the theory in the infinite volume and $\boldsymbol{K}^{(1)}$ is a $3 \times 3$ diagonal matrix of the (complex) fugacities:

$$
\boldsymbol{K}^{(1)}=\left(\begin{array}{ccc}
\mathrm{e}^{+2 \pi \mathrm{i} k} & 0 & 0 \\
0 & 1 & 0 \\
0 & 0 & \mathrm{e}^{-2 \pi \mathrm{i} k}
\end{array}\right)
$$

Following Lüscher [68, 69] (see also ref. [70]), one can derive the explicit formula for the two-particle contribution in eq. (5.18):

$$
\begin{aligned}
\Delta E^{2-\text { particle }}= & m \int_{-\infty}^{\infty} \frac{\mathrm{d} \theta \mathrm{d} \theta^{\prime}}{(2 \pi)^{2}} \cosh (\theta) \mathrm{e}^{-m R \cosh (\theta)-m R \cosh \left(\theta^{\prime}\right)} \\
& \times \operatorname{Tr}_{2}\left[\boldsymbol{K}^{(2)}\left(\pi \boldsymbol{I}^{(2)} \delta\left(\theta-\theta^{\prime}\right)+\mathrm{i} \partial_{\theta} \log \boldsymbol{S}^{(2 \mapsto 2)}\left(\theta-\theta^{\prime}\right)\right)\right],
\end{aligned}
$$

where $\boldsymbol{S}^{(2 \mapsto 2)}$ is the $9 \times 9$ matrix acting in the isotopic component of the two-particle sector, $\boldsymbol{K}^{(2)}=\boldsymbol{K}^{(1)} \otimes \boldsymbol{K}^{(1)}$ and $\boldsymbol{I}^{(2)}$ is the identity matrix.

For future reference let us make a short summary of the properties of the $k$-vacuum energy discussed above. For this purpose it is convenient to introduce the scaling variable

$$
r=m R
$$

and dimensionless scaling function

$$
\mathfrak{F}(r, k)=\frac{R}{\pi}\left(E_{k}^{(\mathrm{vac})}-R \mathcal{E}\right) .
$$

Notice that, $c_{\text {eff }} \equiv-6 \mathfrak{F}(r, k)$ is sometimes interpreted as the effective central charge for the off-critical theory. As $r \rightarrow 0$ our discussion suggests that for $|k|<\frac{1}{n+2}$ and fixed $n$

$$
\mathfrak{F}(r, k)= \begin{cases}-\frac{1}{3}+\frac{4}{n} p^{2}(r)+(n+2) k^{2}+O\left(r^{\frac{4}{n}}\right) & \text { for } \quad n>2 \\ -\frac{1}{3}+\frac{4}{n} p^{2}(r)+(n+2) k^{2}+\delta \mathfrak{F}^{(1-\text { inst })}+O\left(r^{2} p^{2}(r)\right) & \text { for } \quad 0<n<2\end{cases}
$$


Here $p(r)$ is defined as the solution of the quantization condition

$$
-\frac{8 p}{n} \log \left(\frac{r}{8 \pi}\right)+2 \delta\left(p, p_{2}\right)=2 \pi \quad\left(p_{2}<\frac{1}{2}\right)
$$

where $\delta\left(p_{1}, p_{2}\right)$ is given by $(5.11)$, and

$$
\delta \mathfrak{F}^{(1-\mathrm{inst})}=-\frac{r^{2}}{16 \pi^{2}}\left(4 \log \left(\frac{r}{4 \pi}\right)+\pi \cot \left(\frac{\pi n}{2}\right)+2 \gamma_{E}+2 \psi\left(1+\frac{n}{2}\right)+e_{1}(k)\right)
$$

with $e_{1}(k)$ defined in (5.14).

The large $r$-behaviour of the scaling function $\mathfrak{F}(r, k)$ is determined by eqs. (5.18)(5.21). It can be equivalently described by the following formula which is convenient for numerical calculations:

$$
\begin{aligned}
\mathfrak{F}(r, k)= & -\frac{r}{\pi^{2}}(2 c(2 k)+1) K_{1}(r)+\frac{r}{2 \pi^{2}}(2 c(2 k)+1)^{2} K_{1}(2 r)-\frac{2 r}{\pi^{3}}(1+c(2 k)) \\
& \times \int_{-\infty}^{\infty} \mathrm{d} \nu \frac{K_{1-\mathrm{i} \nu}(r) K_{\mathrm{i} \nu}(r)}{\sinh \left(\frac{\pi(n+2) \nu}{2}\right)}\left[2 c(2 k) \sinh \left(\frac{\pi n \nu}{2}\right)-\sinh \left(\frac{\pi(n-2) \nu}{2}\right)\right]+O\left(\mathrm{e}^{-3 r}\right)
\end{aligned}
$$

where $c(x) \equiv \cos (\pi x), K_{s}(z)$ denotes the modified Bessel function of the second order,

$$
K_{s}(z)=\frac{1}{2} \int_{-\infty}^{\infty} \mathrm{d} \theta \mathrm{e}^{s \theta-z \cosh (\theta)}
$$

and the symbol $O\left(\mathrm{e}^{-3 r}\right)$ stands for terms which decay faster than $\mathrm{e}^{-(3-\epsilon) r}$ as $r \rightarrow+\infty$, for any small $\epsilon>0$.

Finally, for $n=0$, the scaling function $\mathfrak{F}(r, k)$ is given explicitly by:

$$
\mathfrak{F}(r, k)=-\frac{r}{2 \pi^{2}} \int_{-\infty}^{\infty} \mathrm{d} \theta \mathrm{e}^{ \pm \theta} \log \left(\frac{\left(1+\mathrm{e}^{2 \pi \mathrm{i} k-r \cosh (\theta)}\right)\left(1+\mathrm{e}^{-2 \pi \mathrm{i} k-r \cosh (\theta)}\right)}{1-\mathrm{e}^{-r \cosh (\theta)}}\right)
$$

Notice that the small $r$-asymptotic (5.24) can not be applied to this exact formula because of the noncommutativity of the limits $r \rightarrow 0$ and $n \rightarrow 0$.

\subsection{NLIE for the $k$-vacuum eigenvalues in the sausage model}

With some experience in working with nonlinear integral equations in integrable QFT, one expects that the generalization of the massless equations to the massive ones requires little effort. For this reason, before exploring the general integrable structures, we make a simple-minded shortcut and guess the NLIE describing the $k$-vacuum eigenvalues in the sausage model. Of course, this route requires careful consistency checks which will be the main subject of our discussion here.

As usual, the major modification required to get the massive NLIE is related to the source terms. In the case under consideration it is not difficult to guess that the sys- 
tem (4.94) should be modified to the following

$$
\begin{aligned}
\varepsilon(\theta-\mathrm{i} \gamma)= & r \sinh (\theta-\mathrm{i} \gamma)-2 \pi k+\int_{-\infty}^{\infty} \frac{\mathrm{d} \theta^{\prime}}{2 \pi \mathrm{i}}\left[G\left(\theta-\theta^{\prime}-2 \mathrm{i} \gamma\right)\left(L\left(\theta^{\prime}-\mathrm{i} \gamma\right)\right)^{*}\right. \\
& \left.-G\left(\theta-\theta^{\prime}\right) L\left(\theta^{\prime}-\mathrm{i} \gamma\right)\right]+\int_{-\infty}^{\infty} \frac{\mathrm{d} \theta^{\prime}}{2 \pi} G_{1}\left(\theta-\theta^{\prime}-\mathrm{i} \gamma\right) \log \left(1+\mathrm{e}^{-\omega\left(\theta^{\prime}\right)}\right) \\
\omega(\theta)= & r \cosh (\theta)+\Im m\left[\int_{-\infty}^{\infty} \frac{\mathrm{d} \theta^{\prime}}{\pi} G_{1}\left(\theta-\theta^{\prime}+\mathrm{i} \gamma\right) L\left(\theta^{\prime}-\mathrm{i} \gamma\right)\right] \\
& -\int_{-\infty}^{\infty} \frac{\mathrm{d} \theta^{\prime}}{\pi} G_{2}\left(\theta-\theta^{\prime}\right) \log \left(1+\mathrm{e}^{-\omega\left(\theta^{\prime}\right)}\right) \\
L(\theta)= & \log \left(1+\mathrm{e}^{-\mathrm{i} \varepsilon(\theta)}\right) .
\end{aligned}
$$

Unlike the massless case, there is no need to supplement these equations by the asymptotic conditions at $\theta \rightarrow-\infty$ - the source terms in (5.30) control the solution's behaviour both at $\theta \rightarrow \pm \infty$. In this subsection we will discuss the $k$-vacuum energy only. Having at hand the formula (C.9) for the vacuum eigenvalue of the conformal local IM $\mathfrak{i}_{1}$, one expects that for the massive case,

$$
\mathfrak{F}(r, k)=\frac{r}{2 \pi^{2}} \int_{-\infty}^{\infty} \mathrm{d} \theta\left( \pm 2 \Im m\left[\mathrm{e}^{ \pm(\theta-\mathrm{i} \gamma)} L(\theta-\mathrm{i} \gamma)\right]-\mathrm{e}^{ \pm \theta} \log \left(1+\mathrm{e}^{-\omega(\theta)}\right)\right)
$$

and this should be valid for both choices of the sign \pm .

Some superficial observations can been made at this point. First we note that the kernels in (5.30) which are given by eqs. (4.96), can be expressed through the two-particle scattering amplitudes for the sausage model. Indeed, using the explicit formulae from appendix E, it is straightforward to check that

$$
\begin{aligned}
G(\theta) & =-\mathrm{i} \partial_{\theta} \log S(\theta), & G_{1}(\theta)=\partial_{\theta} \log t\left(\theta+\frac{\mathrm{i} \pi}{2}\right) \\
G_{2}(\theta) & =-\frac{\mathrm{i}}{8} \partial_{\theta} \log \operatorname{det}\left(S^{(2 \mapsto 2)}(\theta)\right) . &
\end{aligned}
$$

The next observation is that the system (5.30) admits a simple solution for $n=0$. In this case the kernels $G(\theta)$ and $G_{1}(\theta)$ vanish, whereas $G_{2}(\theta)$ turns to be $\pi \delta(\theta)$. This brings the NLIE to the form

$$
\varepsilon(\theta)=r \sinh (\theta)-2 \pi k, \quad \omega(\theta)=r \cosh (\theta)-\log \left(1+\mathrm{e}^{-\omega(\theta)}\right),
$$

and using eq. (5.31), one arrives at (5.29). Furthermore, one can perturbatively solve the NLIE for small $n$, and compare the results to those from the weak coupling expansion based on the dual action (5.8) for the sausage model. We found complete agreement to the first non-trivial order in the expansion.

Much more effort is needed to derive directly from eqs. (5.30), (5.31) the asymptotic formulae (5.24)-(5.27) describing the behaviour of the $k$-vacuum energy at $r \rightarrow 0$ and 

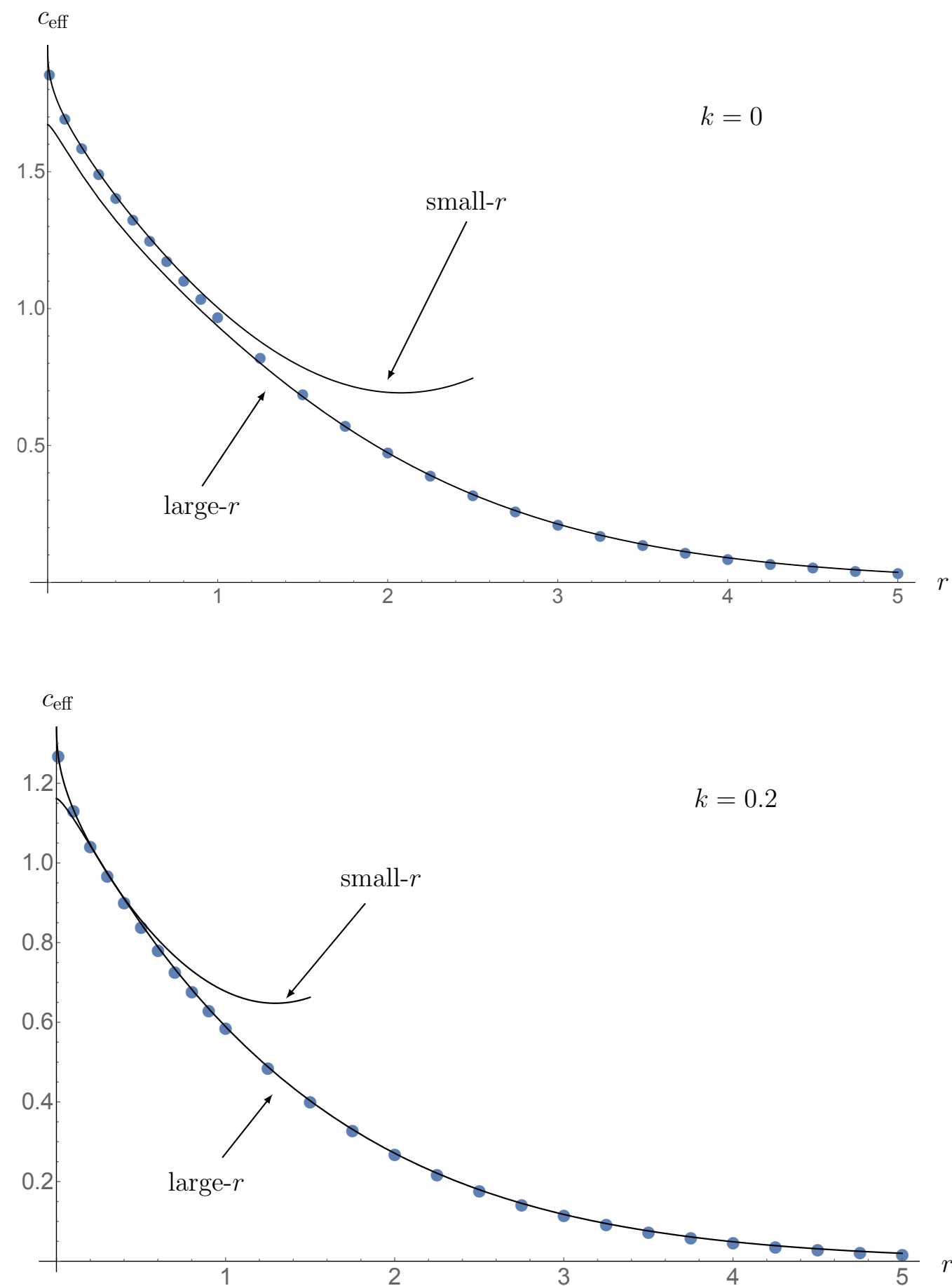

Figure 7. $c_{\text {eff }} \equiv-6 \mathfrak{F}(r, k)(5.23)$ plotted as a function of $r=m R$ for $n=0.5$ with $k=0$ and $k=0.2$. The dots were obtained from the numerical solution of the NLIE (5.30), (5.31). The small$r$ asymptotic comes from (5.24) while "large- $r$ " represents (5.27). For the corresponding numerical data see table 1. 


\begin{tabular}{|c|c|c|c|}
\hline \multicolumn{5}{|c|}{$k=0$} \\
\hline$r=m R$ & $c_{\mathrm{eff}}$ & small- $r$ & large- $r$ \\
\hline $10^{-5}$ & 1.963104810570 & 1.963104810585 & $* * *$ \\
\hline $10^{-4}$ & 1.947406984923 & 1.947406984949 & $* * *$ \\
\hline $10^{-3}$ & 1.919080208689 & 1.919080211060 & $* * *$ \\
\hline 0.01 & 1.859823363074 & 1.859823782193 & $* * *$ \\
\hline 0.10 & 1.698224051017 & 1.698316219415 & $* * *$ \\
\hline 0.20 & 1.589016773023 & 1.589515588521 & $* * *$ \\
\hline 0.40 & 1.409038525289 & 1.411851755076 & $* * *$ \\
\hline 0.60 & 1.250428169768 & 1.258361320834 & 1.1804081 \\
\hline 0.80 & 1.105667568636 & 1.122485892386 & 1.0531828 \\
\hline 1.00 & 0.973032006280 & 1.003537933054 & 0.9358090 \\
\hline 1.50 & 0.691697724451 & 0.785383466436 & 0.6782200 \\
\hline 2.00 & 0.477804027757 & $* * *$ & 0.4735652 \\
\hline 2.50 & 0.322319977959 & $* * *$ & 0.3210983 \\
\hline 3.00 & 0.213430553126 & $* * *$ & 0.2130984 \\
\hline 3.50 & 0.139339032762 & $* * *$ & 0.1392523 \\
\hline 4.00 & 0.089999431197 & $* * *$ & 0.0899774 \\
\hline 4.50 & 0.057660716365 & $* * *$ & 0.0576552 \\
\hline 5.00 & 0.036712137455 & $* * *$ & 0.0367108 \\
\hline \multicolumn{4}{|c|}{$k=0.2$} \\
\hline $10^{-5}$ & 1.364830335405 & 1.364830335417 & $* * *$ \\
\hline $10^{-4}$ & 1.350312382232 & 1.350312382295 & $* * *$ \\
\hline $10^{-3}$ & 1.324531940186 & 1.324531948818 & $* * *$ \\
\hline 0.01 & 1.271882443291 & 1.271883920213 & $* * *$ \\
\hline 0.10 & 1.134046123560 & 1.134350188655 & 1.11141992 \\
\hline 0.20 & 1.044536780739 & 1.046137755580 & 1.04399668 \\
\hline 0.40 & 0.902842376206 & 0.911610323133 & 0.90993729 \\
\hline 0.60 & 0.783720297274 & 0.807969967819 & 0.78900239 \\
\hline 0.80 & 0.679456178786 & 0.729950921532 & 0.68245438 \\
\hline 1.00 & 0.587464397842 & $* * *$ & 0.58894471 \\
\hline 1.50 & 0.402767285740 & $* * *$ & 0.40287319 \\
\hline 2.00 & 0.271053581853 & $* * *$ & 0.27100091 \\
\hline 2.50 & 0.179589431194 & $* * *$ & 0.17955720 \\
\hline 3.00 & 0.117506056343 & $* * *$ & 0.11749384 \\
\hline 3.50 & 0.076124137247 & $* * *$ & 0.07612023 \\
\hline 4.00 & 0.048928716276 & $* * *$ & 0.04892758 \\
\hline 4.50 & 0.031251806913 & $* * *$ & 0.03125149 \\
\hline 5.00 & 0.019860097547 & $* * *$ & 0.01986001 \\
\hline & & & \\
\hline
\end{tabular}

Table 1. The numerical data for $c_{\mathrm{eff}} \equiv-6 \mathfrak{F}(r, k)$ with $n=0.5, k=0$ and 0.2 . The small- $r$ asymptotic was obtained by (5.24) whereas the large- $r$ asymptotic comes from (5.27). 
$r \rightarrow+\infty$. It is, in fact, possible to do this analytically, but here we only present some evidence obtained through the numerical solution of the NLIE system (5.30) (see figure 7 and table 1).

The remarkable feature of the formulae (5.30), (5.31) is that they do not depend explicitly on $n$. Hence, they can be applied to the case with $n$ formally set to infinity, i.e., to the $O(3)$ sigma model. Nothing particularly special happens to the kernels (4.96); as $n \rightarrow \infty$ they just become the rational functions

$$
\begin{aligned}
G(\theta) & =\frac{2 \pi}{(\theta+\mathrm{i} \pi)(\theta-\mathrm{i} \pi)} \\
G_{1}(\theta) & =\frac{4 \pi^{2} \theta}{\left(\theta+\frac{\mathrm{i} \pi}{2}\right)\left(\theta-\frac{\mathrm{i} \pi}{2}\right)\left(\theta+\frac{3 \mathrm{i} \pi}{2}\right)\left(\theta-\frac{3 \mathrm{i} \pi}{2}\right)} \\
G_{2}(\theta) & =G(\theta)-\frac{2 \pi}{(\theta+2 \mathrm{i} \pi)(\theta-2 \mathrm{i} \pi)} .
\end{aligned}
$$

Also the asymptotic formula (5.27) describing the large- $r$ behaviour is, in the $O(3)$ limit,

$$
\begin{aligned}
\mathfrak{F}(r, k)= & -\frac{r}{\pi^{2}}(2 c(2 k)+1) K_{1}(r)+\frac{r}{2 \pi^{2}}(2 c(2 k)+1)^{2} K_{1}(2 r) \\
& -\frac{2 r}{\pi^{3}}(1+c(2 k)) \int_{-\infty}^{\infty} \mathrm{d} \nu K_{1-\mathrm{i} \nu}(r) K_{\mathrm{i} \nu}(r)\left(2 c(2 k) \mathrm{e}^{-\pi|\nu|}-\mathrm{e}^{-2 \pi|\nu|}\right)+O\left(\mathrm{e}^{-3 r}\right) .
\end{aligned}
$$

The situation is much more subtle for the small- $r$ asymptotic. Let us recall that for finite $n$ the asymptotic formula (5.24) can be applied only for $|k|<\frac{1}{n+2}$. This implies that in the limit $n \rightarrow \infty$ the applicability of this formula is restricted to the case $k=0$, and the only information it provides is that $\lim _{r \rightarrow 0} \mathfrak{F}(r, 0)=-\frac{1}{3}$.

As it follows from general perturbative arguments, $\mathfrak{F}(r, 0)$ should admit the power series expansion in terms of the running coupling constant for the $O(3)$ NLSM. It is convenient to choose the RG scheme in which the running coupling $g=g(r)$ satisfies the RG flow equation $[71,72]$

$$
r \frac{\mathrm{d} g}{\mathrm{~d} r}=\frac{g^{2}}{1-g}=g^{2}+g^{3}+\ldots
$$

The solution to this equation which we will use is

$$
g^{-1} \mathrm{e}^{-\frac{1}{g}}=\frac{1}{32 \pi} \mathrm{e}^{\gamma_{E}+1} r .
$$

The funny constant $\frac{1}{32 \pi} \mathrm{e}^{\gamma_{E}+1}=0.048 \ldots$ is chosen following the convention from the works [73-75]. With this choice the gap between the vacuum and the first excited state energies in the $k=0$ sector, $\Delta E_{0}$, admits the perturbative expansion where the term $\propto g^{2}$ is absent: $R \Delta E_{0} /(2 \pi)=g+g^{3}+1.19 g^{4}+O\left(g^{5}\right)$. The small- $r$ behaviour of $\mathfrak{F}(r, 0)$ should admit the asymptotic expansion of the form

$$
\mathfrak{F}(r, 0) \asymp-\frac{1}{3}+a_{1} g(r)+a_{2} g^{2}(r)+a_{3} g^{3}(r)+a_{4} g^{4}(r)+\ldots
$$

The first coefficient in this series is known $a_{1}=\frac{1}{2}$ [14]. All others can, in principle, be calculated within the renormalized perturbation theory for the $O(3)$ NLSM. Instead of 
doing so, we estimated their value by fitting the data obtained from the numerical solution of the NLIE. The fitting suggests that, in all likelihood, $a_{2}=\frac{1}{4}$ and $a_{3} \approx 1$. Also, our numerical results for $k=0$ are in a full agreement with the numerical data quoted in ref. [76]. To the best of our knowledge, the vacuum energies with $0<|k| \leq \frac{1}{2}$ have not been discussed in the literature. ${ }^{14}$ One can expect that for non-zero $k$

$$
\mathfrak{F}(r, k) \asymp a_{0}(k)+a_{1}(k) g(r)+a_{2}(k) g^{2}(r)+a_{3}(k) g^{3}(r)+\ldots
$$

with

$$
a_{0}(k)=-\frac{1}{3}+2|k|(1-|k|) \quad \text { for } \quad|k| \leq \frac{1}{2} .
$$

The last formula can be understood as follows. In the ultraviolet limit the effect of the target space curvature becomes negligible and $\left(-\frac{1}{3}\right)$ here represents the contribution of two massless Goldstones. However, with non-zero $k$, the quasiperiodic boundary condition (1.18) implies the presence of conical singularities at the north and south poles of the 2-sphere. The result of the work [78] for a string propagation on a cone yields eq. (5.40). Our numerical data seems to be in agreement with this prediction. Some of the obtained results are depicted in figure 8 . Note that as $k$ approaches to $\frac{1}{2}$, the calculations for small $r$ require a considerable amount of computational resources.

To complete this subsection, let us return to the case of finite $n$. As has been already mentioned, the quantization condition (5.10) admits the $R$-independent solution $p_{1}=0$ for $p_{2}=\frac{1}{2}$. The latter corresponds to $k=\frac{1}{n+2}$. For this case, as follows from (5.12), the value of the effective central charge at $r=0$ is given by $\frac{2(n-1)}{n+2}$. For integer $n \geq 2$ this coincides with the central charge $c_{n}$ (3.3) of the $\mathbb{Z}_{n}$ parafermions CFT. Based on the results of the work [79], one can expect that the $k$-vacuum energy with $k=\frac{1}{n+2}$ and $n=2,3, \ldots$ coincides with the ground state energy of the non-critical model referred to as $H_{n}^{(0)}$ in [79]. The model can be described by means of the Euclidean action

$$
\mathcal{A}_{H_{n}^{(0)}}=\mathcal{A}_{\mathbb{Z}_{n}}-\lambda \int \mathrm{d}^{2} x\left(\psi^{+} \bar{\psi}^{+}+\psi^{-} \bar{\psi}^{-}\right)
$$

which is the critical action of the $\mathbb{Z}_{n}$ parafermions CFT perturbed by the relevant operator of the scale dimension $d=2-\frac{2}{n}$. According to the work [79], the small- $r$ expansion for the scaling function $\mathfrak{F}$ in this case reads as follows

$$
\mathfrak{F}\left(r, \frac{1}{n+2}\right)=-\frac{1}{6} c_{n}+2 \sum_{j=2}^{\infty} F_{j} r^{\frac{2 j}{n}}+2 F_{(\log )}\left(\frac{r}{2 \pi}\right)^{2} \log (r),
$$

with

$$
F_{(\log )}=\left\{\begin{array}{llll}
-\frac{n-1}{2 n} & \text { for } & n & \text { odd } \\
-\frac{1}{2} & \text { for } & n & \text { even }
\end{array} .\right.
$$

For $n=2$ the model $H_{n}^{(0)}$ coincides with the free theory of a massive Majorana fermion, and therefore

$$
\mathfrak{F}\left(r, \frac{1}{4}\right)=-\frac{r}{2 \pi^{2}} \int_{-\infty}^{\infty} \mathrm{d} \theta \cosh (\theta) \log \left(1+\mathrm{e}^{-r \cosh (\theta)}\right)
$$

This was checked from the numerical solution of the NLIE (5.30).

\footnotetext{
${ }^{14}$ The case $k=\frac{1}{2}$ is of special interest for the application of resurgence theory to the problem of instanton summation in the $\mathbb{C P}^{N-1}$ NLSM [77].
} 


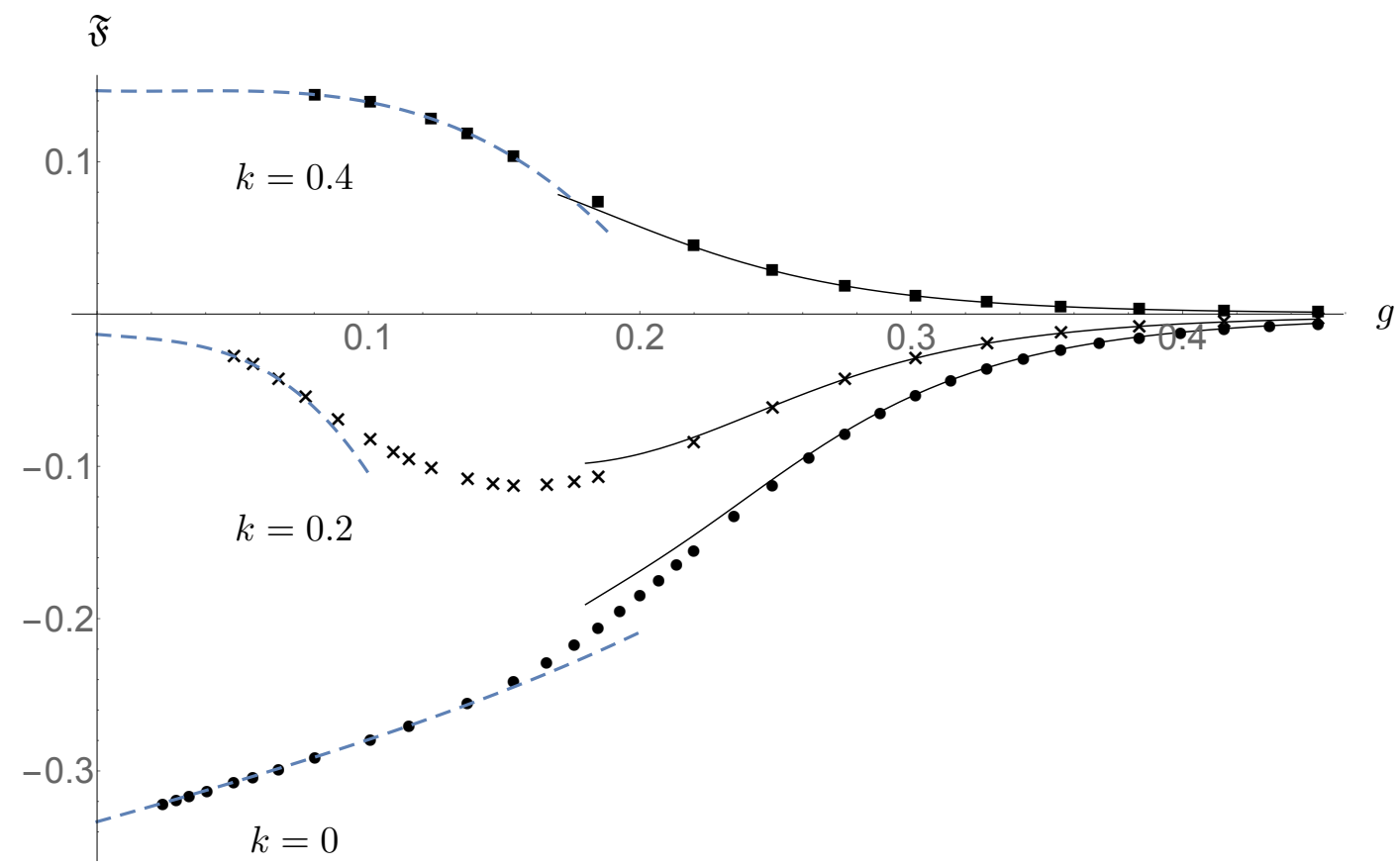

Figure 8. A plot of $\mathfrak{F}(r, k)$ with $k=0,0.2$ and 0.4 as a function of the running coupling constant $g(r)(5.37)$ for the $O(3)$ sigma model. The large- $r$ asymptotics, depicted by the black curves, follow from eq. (5.35). For small $r$ and $k=0.2,0.4$, the numerical data was fitted by a cubic polynomial of the form (5.39) with $a_{0}$ given by (5.40). The result of the fit is represented by the dashed line. For $k=0$ a quartic fit was used (5.38) and the coefficients were found to be $\left(a_{1}, a_{2}, a_{3}\right)=(0.5,0.25,1.0)$. Note that the smallest value of the running coupling that we reached is $g=0.0242 \ldots$ (for $k=0$ ), whereas the largest value is $g=0.449 \ldots$ These correspond to $r=10^{-15}$ and $r=5$, respectively.

\section{$5.3 \quad \mathbb{A}, \mathbb{B}$ and $\mathbb{T}$}

We are now ready to discuss the general integrable structures in the quantum sausage model. In fact, they are almost identical to those from the cigar/sine-Liouville CFT. We will place a special emphasis on the aspects of the integrable structures which are related to the presence of the finite correlation length in the theory.

Recall that the sine-Liouville model possesses an infinite set of involutive local IM. At the end of section 2 we mentioned only the integrals of the left chirality. Of course, there are also the "right" local IM, so that the full commuting set is $\left\{\mathfrak{i}_{2 j-1}, \overline{\mathfrak{i}}_{2 j-1}\right\}_{j=1}^{\infty}$. Remarkably (see, e.g., [44] for details), all the local IM are invariant under the reflection $\varphi \mapsto-\varphi$, and therefore they can be interpreted as the local IM for both theories (5.6) and (5.7). This observation suggests that the quantum sausage NLSM possesses the infinite set of local IM $\left\{\mathbb{I}_{2 j-1}, \overline{\mathbb{I}}_{2 j-1}\right\}_{j=1}^{\infty}$ which can be thought of, in a certain sense, as a deformation of the conformal one [80]. In particular

$$
\mathbb{I}_{2 j-1}=\int_{0}^{R} \frac{\mathrm{d} x}{2 \pi}\left(\sum_{l+m=j} C_{l m}^{(j)}\left(\partial_{+} \varphi\right)^{2 l}\left(\partial_{+} \vartheta\right)^{2 m}+\ldots\right)
$$

and similar for $\overline{\mathbb{I}}_{2 j-1}$ with $\partial_{+}$replaced by $\partial_{-}$. Here we use the light cone variables $x^{ \pm}=$ 
$x^{0} \pm x^{1}$, the constants $C_{l m}^{(j)}$ are the same as in eqs. (2.74)-(2.76), and the dots stand for monomials which include higher derivatives of $\varphi$ and $\vartheta$, as well as terms proportional to powers of $\mathcal{M}$. It should be emphasized that the $\varphi$ and $\vartheta$ in (5.45) are local fields whose dynamics are governed by the dual action (5.8) and, if considering the neutral sector of the model, the periodic boundary conditions (5.9). A special rôle belongs to the integrals

$$
\begin{aligned}
& \mathbb{I}_{1}=\int_{0}^{R} \frac{\mathrm{d} x}{2 \pi}\left(\left(\partial_{+} \varphi\right)^{2}+\left(\partial_{+} \vartheta\right)^{2}-4 \mathcal{M} \cosh (\sqrt{n} \varphi) \cos (\sqrt{n+2} \vartheta)\right) \\
& \overline{\mathbb{I}}_{1}=\left(\partial_{+} \mapsto \partial_{-}\right),
\end{aligned}
$$

whose sum, $\mathbb{H}_{R}=\mathbb{I}_{1}+\overline{\mathbb{I}}_{1}$, and difference, $\mathbb{P}_{R}=\mathbb{I}_{1}-\overline{\mathbb{I}}_{1}$, coincide with the Hamiltonian and the total momentum, respectively. It is expected that the common eigenvectors of $\left\{\mathbb{I}_{2 j-1}, \overline{\mathbb{I}}_{2 j-1}\right\}_{j=1}^{\infty}$ form a basis in each invariant subspace $\mathcal{H}_{k}^{(K)}$ of the Hilbert space of the sausage NLSM. Let's denote the corresponding $k$-vacuum eigenvalues by $\left\{\mathfrak{I}_{2 j-1}, \overline{\mathfrak{I}}_{2 j-1}\right\}_{j=1}^{\infty}$. For the $k$-vacuum the total momentum is zero, so

$$
\mathfrak{I}_{1}=\overline{\mathfrak{I}}_{1}=\frac{1}{2} \mathcal{E} R+\frac{\pi}{2 R} \mathfrak{F}(r, k) .
$$

This relation together with (5.31), allows one to express the vacuum eigenvalues of $\mathbb{I}_{1}$ and $\overline{\mathbb{I}}_{1}$ in terms of the solution to the NLIE (5.30). It is not difficult to find similar expressions for the other local IM (to be compared with formulae (C.9) from appendix C):

$$
\begin{aligned}
& \mathfrak{I}_{2 j-1}=\left(\frac{m}{4}\right)^{2 j-1} \int_{-\infty}^{\infty} \frac{\mathrm{d} \theta}{\pi}\left((-1)^{j} \mathrm{e}^{+(2 j-1) \theta} \log \left(1+\mathrm{e}^{-\omega(\theta)}\right)+2 \Im m\left[\mathrm{e}^{+(2 j-1)(\theta-\mathrm{i} \gamma)} L(\theta-\mathrm{i} \gamma)\right]\right) \\
& \overline{\mathfrak{I}}_{2 j-1}=\left(\frac{m}{4}\right)^{2 j-1} \int_{-\infty}^{\infty} \frac{\mathrm{d} \theta}{\pi}\left((-1)^{j} \mathrm{e}^{-(2 j-1) \theta} \log \left(1+\mathrm{e}^{-\omega(\theta)}\right)-2 \Im m\left[\mathrm{e}^{-(2 j-1)(\theta-\mathrm{i} \gamma)} L(\theta-\mathrm{i} \gamma)\right]\right)
\end{aligned}
$$

For $r \ll 1$, similar to formula (5.24) for $\mathfrak{F}(r, k)$, the vacuum eigenvalues of the higher spin local IM can be approximated by

$$
\mathfrak{I}_{2 j-1}=\overline{\mathfrak{I}}_{2 j-1} \approx\left(\frac{2 \pi}{R}\right)^{2 j-1} i_{2 j-1}\left(p(r), \frac{1}{2}(n+2) k\right) .
$$

Here, $i_{2 j-1}\left(p_{1}, p_{2}\right)$ are the vacuum eigenvalues of the chiral local IM $\mathfrak{i}_{2 j-1}$ and $p=p(r)$ is the solution of eq. (5.25). Table 2 demonstrates the quality of this approximation for the first few local IM.

Note that $\mathfrak{I}_{2 j-1}=\overline{\mathfrak{I}}_{2 j-1}$ for any $j=1,2, \ldots$ These relations can be easily understood since the model (5.8) is $\mathcal{P}$-invariant and that under the parity transformation

$$
\mathcal{P} \mathbb{I}_{2 j-1} \mathcal{P}=\overline{\mathbb{I}}_{2 j-1} .
$$

Another important global symmetry is $\mathcal{C}$-invariance. Acting on the local fields it flips the sign of $\vartheta$ while keeping $\varphi$ unchanged. All the local IM are $\mathcal{C}$-invariant operators, i.e.,

$$
\mathcal{C} \mathbb{I}_{2 j-1} \mathcal{C}=\mathbb{I}_{2 j-1}, \quad C \overline{\mathbb{I}}_{2 j-1} C=\overline{\mathbb{I}}_{2 j-1} .
$$




\begin{tabular}{|c|c|c|}
\hline$r=m R$ & $\left(\frac{R}{2 \pi}\right)^{3} \Im_{3}$ & $i_{3}\left(p(r), p_{2}\right)$ \\
\hline $10^{-1}$ & $3.45716396595 \times 10^{-4}$ & $3.45832599760 \times 10^{-4}$ \\
\hline $10^{-3}$ & $3.81476584833 \times 10^{-4}$ & $3.81476594313 \times 10^{-4}$ \\
\hline $10^{-5}$ & $3.92737566343 \times 10^{-4}$ & $3.92737566334 \times 10^{-4}$ \\
\hline$r=m R$ & $\left(\frac{R}{2 \pi}\right)^{5} \Im_{5}$ & $i_{5}\left(p(r), p_{2}\right)$ \\
\hline $10^{-1}$ & $-2.6148731 \times 10^{-5}$ & $-2.6151868 \times 10^{-5}$ \\
\hline $10^{-3}$ & $-2.8189874 \times 10^{-5}$ & $-2.8189869 \times 10^{-5}$ \\
\hline $10^{-5}$ & $-2.8833109 \times 10^{-5}$ & $-2.8833124 \times 10^{-5}$ \\
\hline
\end{tabular}

Table 2. The vacuum eigenvalues of the first two higher spin local IM in the sausage model for $n=1$ and $p_{2}=\frac{3}{2} k=\frac{5}{13}$. The numerical values were calculated from the solution of the NLIE and formula (5.48). The last column gives the vacuum eigenvalues of the chiral local IM, $i_{3}\left(p(r), p_{2}\right)$ and $i_{5}\left(p(r), p_{2}\right)$, where $p(r)$ is the solution to the quantization condition (5.25). The limiting values at $r=0$ are given by $i_{3}\left(0, p_{2}\right)=\frac{39031}{95964960}=4.067 \ldots \times 10^{-4}, i_{5}\left(0, p_{2}\right)=-\frac{137442779}{4638370376640}=$ $-2.963 \ldots \times 10^{-5}$. Explicit expressions for $i_{3}$ and $i_{5}$ can be found in ref. [42].

Since $C \mid$ vac $\rangle_{k}=|\operatorname{vac}\rangle_{-k}$, this explains the fact that the vacuum eigenvalues $\mathfrak{I}_{2 j-1}$ are even functions of $k$. The last discrete symmetry that we shall consider is the invariance of the dual action (5.8) w.r.t. the transformation

$$
\vartheta(r, t) \mapsto \mathbb{U} \vartheta(t, x) \mathbb{U}^{-1}=\vartheta(t, x)+\frac{2 \pi}{\sqrt{n+2}},
$$

where the unitary operator $\mathbb{U}$ is the Flouquet-Bloch operator which is just a constant phase factor when it acts on the subspace $\mathcal{H}_{k}^{(K)}$ :

$$
\mathbb{U} \mathcal{H}_{k}^{(K)}=\mathrm{e}^{2 \pi \mathrm{i} k} \mathcal{H}_{k}^{(K)} .
$$

Of course, $\left[\mathbb{U}, \mathbb{I}_{2 j-1}\right]=\left[\mathbb{U}, \overline{\mathbb{I}}_{2 j-1}\right]=0$.

Together with the local IM, the sausage model possesses the set of dual nonlocal IM $\left\{\mathbb{S}_{j}, \overline{\mathbb{S}}_{j}\right\}_{j=1}^{\infty}$, which again can be understood as a deformation of the corresponding conformal set. In contrast to the local IM, they are not $\mathcal{C}$-invariant operators. Instead they satisfy the relations

$$
C P \mathbb{S}_{j} C P=\overline{\mathbb{S}}_{j}
$$

This implies that the analog of eq. (5.49) for the set $\left\{\mathfrak{S}_{j}, \overline{\mathfrak{S}}_{j}\right\}_{j=1}^{\infty}$ of $k$-vacuum eigenvalues of the dual nonlocal IM reads as follows

$$
\mathfrak{S}_{j} \approx\left(\frac{2 \pi}{R}\right)^{\frac{2 j}{n+2}} \tilde{s}_{j}\left(p(r),+\frac{1}{2}(n+2) k\right), \quad \overline{\mathfrak{S}}_{j} \approx\left(\frac{2 \pi}{R}\right)^{\frac{2 j}{n+2}} \tilde{s}_{j}\left(p(r),-\frac{1}{2}(n+2) k\right) .
$$

Similarly as for the local IM, the vacuum eigenvalues of the dual nonlocal IM can be 


\begin{tabular}{|c|c|c|c|c|}
\hline$r=m R$ & $\left(\frac{R}{2 \pi}\right)^{\frac{2}{n+2}} \mathfrak{S}_{1}$ & $\tilde{s}_{1}\left(p(r), p_{2}\right)$ & $\left(\frac{R}{2 \pi}\right)^{\frac{2}{n+2}} \overline{\mathfrak{S}}_{1}$ & $\tilde{s}_{1}\left(p(r),-p_{2}\right)$ \\
\hline $10^{-1}$ & 0.01936579 & 0.01877110 & 0.13129934 & 0.12726735 \\
\hline $10^{-3}$ & 0.03374979 & 0.03374058 & 0.22882227 & 0.22875988 \\
\hline $10^{-5}$ & 0.04003149 & 0.04003134 & 0.27141198 & 0.27141097 \\
\hline
\end{tabular}

\begin{tabular}{|c|c|c|}
\hline$r=m R$ & $\left(\frac{r}{8 \pi}\right)^{2 k} \mathfrak{S}$ & $S\left(p_{2} \mid \mathrm{i} p(r)\right)$ \\
\hline $10^{-1}$ & 0.35644580 & 0.35731884 \\
\hline $10^{-3}$ & 0.24462688 & 0.24463961 \\
\hline $10^{-5}$ & 0.19673087 & 0.19673105 \\
\hline
\end{tabular}

Table 3. The vacuum eigenvalues of the dual nonlocal IM $\mathbb{S}_{1}, \overline{\mathbb{S}}_{1}$ and $\mathbb{S}$ for $n=\frac{9}{2}$ and $p_{2}=\frac{13}{4} k=$ $\frac{5}{13}$. Eq. (5.56) was used to find the numerical values of $\mathfrak{S}_{1}, \overline{\mathfrak{S}}_{1}$ from the solution to the NLIE, whereas the corresponding formula for $\mathfrak{S}$ is $(5.59)$. The vacuum eigenvalues $\tilde{s}_{1}\left(p_{1}, p_{2}\right)$ of the chiral dual nonlocal IM are given by (4.84) and the expression for $S\left(p_{2} \mid q\right)$ is found in (5.61). Finally, $p(r)$ is the solution of the quantization condition (5.25).

expressed through the solution of the NLIE:

$$
\begin{aligned}
& \mathfrak{S}_{j}=\frac{2}{n+2}\left(\frac{m}{4}\right)^{\frac{2 j}{n+2}} \int_{-\infty}^{\infty} \frac{\mathrm{d} \theta}{\pi}\left(\sin \left(\frac{\pi j}{n+2}\right) \mathrm{e}^{+\frac{2 j \theta}{n+2}} \log \left(1+\mathrm{e}^{-\omega(\theta)}\right)-\Im m\left[\mathrm{e}^{+\frac{2 j(\theta-\mathrm{i} \gamma)}{n+2}} L(\theta-\mathrm{i} \gamma)\right]\right) \\
& \overline{\mathfrak{S}}_{j}=\frac{2}{n+2}\left(\frac{m}{4}\right)^{\frac{2 j}{n+2}} \int_{-\infty}^{\infty} \frac{\mathrm{d} \theta}{\pi}\left(\sin \left(\frac{\pi j}{n+2}\right) \mathrm{e}^{-\frac{2 j \theta}{n+2}} \log \left(1+\mathrm{e}^{-\omega(\theta)}\right)+\Im m\left[\mathrm{e}^{-\frac{2 j(\theta-\mathrm{i} \gamma)}{n+2}} L(\theta-\mathrm{i} \gamma)\right]\right)
\end{aligned}
$$

In table 3 we present numerical data illustrating formulae (5.55) for $\mathfrak{S}_{1}$ and $\overline{\mathfrak{S}}_{1}$.

We are now able to synthesize our study of the quantum sausage model in the form of the following conjecture. It is expected that the theory possesses the operators $\mathbb{A}(\theta), \mathbb{B}(\theta)$ and $\mathbb{T}(\theta)$ satisfying the set of conditions listed on the following page.

(i) Commutativity: $\quad\left[\mathbb{A}(\theta), \mathbb{A}\left(\theta^{\prime}\right)\right]=\left[\mathbb{B}(\theta), \mathbb{B}\left(\theta^{\prime}\right)\right]=\left[\mathbb{A}(\theta), \mathbb{B}\left(\theta^{\prime}\right)\right]$

(ii) Analyticity: the operators $\mathbb{A}(\theta), \mathbb{B}(\theta)$ and $\mathbb{T}(\theta)$ are entire functions of $\theta$.

(iii) Global symmetries:

$$
\begin{aligned}
\mathcal{P} \mathbb{A}(\theta) \mathcal{C P} & =\mathbb{A}(-\theta), & C \mathcal{P}(\theta) \mathcal{C P}=\mathbb{B}(-\theta) \\
\mathcal{P} \mathbb{T}(\theta) \mathcal{P} & =\mathbb{T}(-\theta), & \mathcal{T}(\theta) \mathcal{C}=\mathbb{T}(\theta) \\
{[\mathbb{U}, \mathbb{A}(\theta)] } & =[\mathbb{U}, \mathbb{B}(\theta)]=[\mathbb{U}, \mathbb{T}(\theta)]=0 &
\end{aligned}
$$

(iv) (Quasi)periodicity: $\mathbb{B}(\theta+\mathrm{i} \pi)=\mathbb{U} \mathbb{B}(\theta-\mathrm{i} \pi), \quad \mathbb{T}(\theta+\mathrm{i} \pi n)=\mathbb{T}(\theta)$ 
(v) Hermiticity: $\quad \mathbb{A}^{\dagger}(\theta)=\mathbb{A}\left(\theta^{*}\right), \quad \mathbb{B}^{\dagger}(\theta)=\mathbb{B}\left(\theta^{*}\right), \quad \mathbb{T}^{\dagger}(\theta)=\mathbb{T}\left(\theta^{*}\right)$

(vi) Functional relation:

$$
\begin{aligned}
\mathbb{A}(\theta & \left.-\frac{\mathrm{i} \pi(n+1)}{2}\right) \mathbb{A}\left(\theta+\frac{\mathrm{i} \pi(n+1)}{2}\right)-\mathbb{A}\left(\theta-\frac{\mathrm{i} \pi(n-1)}{2}\right) \mathbb{A}\left(\theta+\frac{\mathrm{i} \pi(n-1)}{2}\right) \\
& =\mathbb{B}\left(\theta-\frac{\mathrm{i} \pi}{2}\right) \mathbb{B}\left(\theta+\frac{\mathrm{i} \pi}{2}\right)
\end{aligned}
$$

(vii) $\boldsymbol{T}-\boldsymbol{Q}$ relation: $\quad \mathbb{T}\left(\theta+\frac{\mathrm{i} \pi n}{2}\right) \mathbb{A}(\theta)=\mathbb{U}^{-\frac{1}{2}} \mathbb{A}(\theta+\mathrm{i} \pi)+\mathbb{U}^{+\frac{1}{2}} \mathbb{A}(\theta-\mathrm{i} \pi)$

(viii) Asymptotic behaviour of $\mathbb{A}(\theta)$ :

$$
\mathbb{A}(\theta) \asymp \mathbb{S}^{ \pm \frac{1}{2}} \exp \left(-\frac{r \cosh (\theta)}{4 \sin \left(\frac{\pi n}{2}\right)}\right) \exp \left(-a^{( \pm)}(\theta)\right) \quad \text { as } \quad \Re e(\theta) \rightarrow \pm \infty
$$

with $|\Im m(\theta)|<\frac{\pi}{2}(n+2)$, and

$$
a^{(+)}(\theta)=\sum_{j=1}^{\infty} \mathbb{S}_{j}\left(\frac{m}{4} \mathrm{e}^{+\theta}\right)^{-\frac{2 j}{n+2}}, \quad a^{(-)}(\theta)=\sum_{j=1}^{\infty} \overline{\mathbb{S}}_{j}\left(\frac{m}{4} \mathrm{e}^{-\theta}\right)^{-\frac{2 j}{n+2}}
$$

(ix) Asymptotic behaviour of $\mathbb{B}(\boldsymbol{\theta})$ :

$$
\mathbb{B}(\theta) \asymp \mathbb{S}^{ \pm \frac{1}{2}} \exp \left(-\frac{r \theta \sinh (\theta)}{2 \pi}\right) \exp \left(-b^{( \pm)}(\theta)\right) \quad \text { as } \quad \Re e(\theta) \rightarrow \pm \infty
$$

with $|\Im m(\theta)|<\pi$, and

$$
\begin{aligned}
& b^{(+)}(\theta)=\left(\mathbb{I}_{1}-\frac{1}{2} \mathcal{E} R\right) \frac{4}{m} \mathrm{e}^{-\theta}+\sum_{j=1}^{\infty}\left(\mathbb{I}_{2 j+1}\left(\frac{m}{4} \mathrm{e}^{+\theta}\right)^{-1-2 j}+\mathbb{S}_{j}\left(\frac{m}{4} \mathrm{e}^{+\theta}\right)^{-\frac{2 j}{n+2}}\right) \\
& b^{(-)}(\theta)=\left(\overline{\mathbb{I}}_{1}-\frac{1}{2} \mathcal{E} R\right) \frac{4}{m} \mathrm{e}^{+\theta}+\sum_{j=1}^{\infty}\left(\overline{\mathbb{I}}_{2 j+1}\left(\frac{m}{4} \mathrm{e}^{-\theta}\right)^{-1-2 j}+\overline{\mathbb{S}}_{j}\left(\frac{m}{4} \mathrm{e}^{-\theta}\right)^{-\frac{2 j}{n+2}}\right)
\end{aligned}
$$

(x) Zeroes: let $\mathfrak{A}^{(\psi)}(\theta), \mathfrak{B}^{(\psi)}(\theta), \mathrm{e}^{2 \pi \mathrm{i} k}$ be the eigenvalues of the operators $\mathbb{A}(\theta), \mathbb{B}(\theta), \mathbb{U}$, respectively, corresponding to a common eigenvector $|\psi\rangle$. If $\theta_{j}$ is a zero of $\mathfrak{B}^{(\psi)}(\theta)$, then

$$
\exp \left(-\frac{\mathrm{i}}{2} r \sinh \left(\theta_{j}\right)-2 \pi \mathrm{i} k\right) \frac{\mathfrak{A}^{(\psi)}\left(\theta_{j}-\mathrm{i} \pi-\frac{\mathrm{i} \pi n}{2}\right)}{\mathfrak{A}^{(\psi)}\left(\theta_{j}-\mathrm{i} \pi+\frac{\mathrm{i} \pi n}{2}\right)}=-1 .
$$

All zeroes of $\mathfrak{B}^{(\psi)}(\theta)$ are simple and accumulate towards infinity along the lines $\Im m(\theta)=\pi(\bmod 2 \pi)$.

Clearly, the conjectured properties of $\mathbb{A}(\theta), \mathbb{B}(\theta)$ and $\mathbb{T}(\theta)$ are inspired by those of their chiral counterparts $\alpha_{+}(\theta), \beta_{+}(\theta)$ and $\tau(\lambda)$ and the global symmetries of the model. Unlike the chiral case, the subscript was not included in the notation of operators $\mathbb{A}$ and $\mathbb{B}$. It can be restored by setting $\mathbb{A}_{+} \equiv \mathbb{A}$ and $\mathbb{B}_{+} \equiv \mathbb{B}$. The properties of the $\mathcal{C}$-conjugated operators $\mathbb{A}_{-} \equiv$ 
$\mathcal{C} \mathbb{C}, \mathbb{B}_{-} \equiv \mathcal{C} \mathbb{B} C$ can be easily deduced from (i)-(x). Perhaps only the $\theta$-independent operator $\mathbb{S}$, which appears in the large- $\theta$ asymptotic expansions (viii) and (ix), requires some elucidations. Before presenting them, let us first discuss the vacuum eigenvalues of $\mathbb{A}(\theta)$ and $\mathbb{B}(\theta)$. The obvious counterparts to the formulae (C.6), (C.7) from appendix $\mathrm{C}$ read as

$$
\begin{aligned}
\log \mathfrak{A}(\theta)= & -\frac{r \cosh (\theta)}{4 \sin \left(\frac{\pi n}{2}\right)}+\int_{-\infty}^{\infty} \frac{\mathrm{d} \theta^{\prime}}{2 \pi \mathrm{i}}\left[F_{1}\left(\theta-\theta^{\prime}+\mathrm{i} \gamma\right) L\left(\theta^{\prime}-\mathrm{i} \gamma\right)\right. \\
& \left.-F_{1}\left(\theta-\theta^{\prime}-\mathrm{i} \gamma\right)\left(L\left(\theta^{\prime}-\mathrm{i} \gamma\right)\right)^{*}\right]+\int_{-\infty}^{\infty} \frac{\mathrm{d} \theta^{\prime}}{\pi} F_{2}\left(\theta-\theta^{\prime}\right) \log \left(1+\mathrm{e}^{-\omega\left(\theta^{\prime}\right)}\right)
\end{aligned}
$$

where $|\Im m(\theta)|<\frac{\pi}{2}(n+2)-\gamma$, and

$$
\begin{aligned}
\log \mathfrak{B}(\theta)= & -\frac{r \theta \sinh (\theta)}{2 \pi}+\int_{-\infty}^{\infty} \frac{\mathrm{d} \theta^{\prime}}{2 \pi \mathrm{i}}\left[F_{3}\left(\theta-\theta^{\prime}+\mathrm{i} \gamma\right) L\left(\theta^{\prime}-\mathrm{i} \gamma\right)\right. \\
& \left.-F_{3}\left(\theta-\theta^{\prime}-\mathrm{i} \gamma\right)\left(L\left(\theta^{\prime}-\mathrm{i} \gamma\right)\right)^{*}\right]-\int_{-\infty}^{\infty} \frac{\mathrm{d} \theta^{\prime}}{\pi} F_{4}\left(\theta-\theta^{\prime}\right) \log \left(1+\mathrm{e}^{-\omega\left(\theta^{\prime}\right)}\right),
\end{aligned}
$$

with $|\Im m(\theta)|<\pi-\gamma$. Now $\varepsilon(\theta)$ and $\omega(\theta)$ solve the massive NLIE (5.30), (4.96) and the explicit form of the functions $F_{i}(\theta)$ are given in (C.8). It is easy to see that these formulae combined with the asymptotics (viii) and (ix), yield the expressions (5.48) and (5.56) for the vacuum eigenvalues of the local and dual nonlocal IM. Notice that the term $(-k \theta)$ is absent in (5.57), (5.58) compared with the analogous formulae (C.6), (C.7). This is consistent with the absence of the factor $\mathrm{e}^{-k \theta}$ in the asymptotics (viii) and (ix) compared with the corresponding eqs. (4.70), (4.71) and (4.74) for the chiral case. In connection with this, note that the operator $\hat{k}$ is ill defined and only its exponent $\mathbb{U}=\exp (2 \pi \mathrm{i} \hat{k})$ makes sense in the massive theory.

In the next subsection we will point out that the eigenvalues of the operator $\mathbb{S}$ play a special rôle in the ODE/IQFT correspondence. Eqs. (5.57), (5.58) predict that in the case of the $k$-vacuum states, its eigenvalue is given by

$$
\mathfrak{S}=\exp \left(\frac{2}{n+2} \int_{-\infty}^{\infty} \frac{\mathrm{d} \theta}{\pi} \Im m(L(\theta-\mathrm{i} \gamma))\right)
$$

The small- $r$ behaviour of $\mathfrak{S}$ is given by a formula similar to (5.49), (5.55) (see table 3):

$$
\mathfrak{S} \approx\left(\frac{8 \pi}{r}\right)^{2 k} S\left(\frac{1}{2}(n+2) k \mid \mathrm{i} p(r)\right)
$$

where

$$
S\left(p_{2} \mid q\right)=(n+2)^{\frac{4 p_{2}}{n+2}} \frac{\Gamma\left(\frac{1}{2}+p_{2}+q\right) \Gamma\left(\frac{1}{2}+p_{2}-q\right)}{\Gamma\left(\frac{1}{2}-p_{2}+q\right) \Gamma\left(\frac{1}{2}-p_{2}-q\right)} \frac{\Gamma\left(1-2 p_{2}\right)}{\Gamma\left(1+2 p_{2}\right)} \frac{\Gamma\left(1+\frac{2 p_{2}}{n+2}\right)}{\Gamma\left(1-\frac{2 p_{2}}{n+2}\right)} .
$$

Notice that $S\left(p_{2} \mid \mathrm{i} p_{1}\right)$ can be expressed in terms of the vacuum eigenvalues (4.85) of the operators $b_{ \pm}$defined in (4.61): $S\left(p_{2} \mid \mathrm{i} p_{1}\right)=b_{-}^{(\text {vac })}\left(p_{1}, p_{2}\right) / b_{+}^{(\text {vac })}\left(p_{1}, p_{2}\right)$.

The operator $\mathbb{T}(\theta)$ is the transfer-matrix in the sausage model - the quantum counterpart of the Wilson loop (1.1) which was the starting point of this paper. By means of the 
$T-Q$ equation (vii) it is expressed in terms of the operator $\mathbb{A}(\theta)$ and, of course, commutes with both $\mathbb{A}$ and $\mathbb{B}$ for any values of the spectral parameter $\theta$. We did not include the formula which described its large- $\theta$ asymptotic in the list (i) $-(\mathrm{x})$ since it is an immediate consequence of the $T-Q$ equation and the asymptotic (viii) for $\mathbb{A}(\theta)$. Notice that unlike for the Toda-type theory, the transfer-matrix in the sausage model does not generate the local IM through its asymptotic expansion.

Finally we can turn to the case of the $O(3)$ NLSM. There is no reason to expect that the $n \rightarrow \infty$ limit is problematic for the operator $\mathbb{B}(\theta)$. Introduce the notation

$$
\mathbb{B}_{\infty}(\theta)=\lim _{n \rightarrow \infty} \mathbb{B}(\theta) .
$$

Using eqs. (5.58), (C.8), one finds the relation which expresses its vacuum eigenvalue in terms of the solution to the NLIE (5.30) with the kernels (5.34):

$$
\begin{aligned}
\log \mathfrak{B}_{\infty}(\theta)= & -\frac{r \theta \sinh (\theta)}{2 \pi}+\int_{-\infty}^{\infty} \frac{\mathrm{d} \theta^{\prime}}{2 \pi \mathrm{i}}\left[f_{3}\left(\theta-\theta^{\prime}+\mathrm{i} \gamma\right) L\left(\theta^{\prime}-\mathrm{i} \gamma\right)\right. \\
& \left.-f_{3}\left(\theta-\theta^{\prime}-\mathrm{i} \gamma\right)\left(L\left(\theta^{\prime}-\mathrm{i} \gamma\right)\right)^{*}\right]-\int_{-\infty}^{\infty} \frac{\mathrm{d} \theta^{\prime}}{\pi} f_{4}\left(\theta-\theta^{\prime}\right) \log \left(1+\mathrm{e}^{-\omega\left(\theta^{\prime}\right)}\right)
\end{aligned}
$$

where $|\Im m(\theta)|<\pi-\gamma$, and

$$
f_{3}(\theta)=\frac{1}{\theta}-\frac{1}{\sinh (\theta)}, \quad f_{4}(\theta)=\frac{\pi}{2\left(\theta+\frac{\mathrm{i} \pi}{2}\right)\left(\theta-\frac{\mathrm{i} \pi}{2}\right)}-\frac{1}{2 \cosh (\theta)} .
$$

The situation with the operators $\mathbb{A}(\theta)$ and $\mathbb{T}(\theta)$ is slightly more delicate. In this case, one can expect that the following limits exist

$$
\begin{aligned}
& \mathbb{A}_{\infty}(\theta)=\lim _{n \rightarrow \infty} \mathbb{A}\left(\theta-\frac{\mathrm{i} \pi n}{2}\right) \exp \left(\frac{1}{4} r \cot \left(\frac{\pi n}{2}\right) \cosh (\theta)\right) \\
& \mathbb{T}_{\infty}(\theta)=\lim _{n \rightarrow \infty} \mathbb{T}(\theta) \exp \left(-\frac{1}{2} r \cot \left(\frac{\pi n}{2}\right) \cosh (\theta)\right)
\end{aligned}
$$

and the limiting operators satisfy the $T-Q$ equation in the form

$$
\mathbb{T}_{\infty}(\theta) \mathbb{A}_{\infty}(\theta)=\mathbb{U}^{-\frac{1}{2}} \mathbb{A}_{\infty}(\theta+\mathrm{i} \pi)+\mathbb{U}^{+\frac{1}{2}} \mathbb{A}_{\infty}(\theta-\mathrm{i} \pi)
$$

(recall that in the sector $\mathcal{H}_{k}^{(K)}$ the operator $\mathbb{U}^{+\frac{1}{2}}$ becomes just a phase factor $(-1)^{K-1} \mathrm{e}^{\mathrm{i} \pi k}$ ). With eqs. (5.57), (C.8), it is easy to see that

$$
\begin{aligned}
\log \mathfrak{A}_{\infty}(\theta)= & \frac{\mathrm{i}}{4} r \sinh (\theta)+\int_{-\infty}^{\infty} \frac{\mathrm{d} \theta^{\prime}}{2 \pi \mathrm{i}}\left[f_{1}\left(\theta-\theta^{\prime}+\mathrm{i} \gamma\right) L\left(\theta^{\prime}-\mathrm{i} \gamma\right)\right. \\
& \left.-f_{1}\left(\theta-\theta^{\prime}-\mathrm{i} \gamma\right)\left(L\left(\theta^{\prime}-\mathrm{i} \gamma\right)\right)^{*}\right]+\int_{-\infty}^{\infty} \frac{\mathrm{d} \theta^{\prime}}{\pi} f_{2}\left(\theta-\theta^{\prime}\right) \log \left(1+\mathrm{e}^{-\omega\left(\theta^{\prime}\right)}\right)
\end{aligned}
$$

with $\Im m(\theta)<\pi-\gamma$,

$$
f_{1}(\theta)=\frac{1}{\theta+\mathrm{i} \pi}, \quad f_{2}(\theta)=-\frac{\pi}{2\left(\theta+\frac{3 \mathrm{i} \pi}{2}\right)\left(\theta+\frac{\mathrm{i} \pi}{2}\right)},
$$

and $\varepsilon(\theta), \omega(\theta)$ are defined through the solution of the NLIE (5.30), (5.34). 


\subsection{ODE/IQFT for the sausage model}

In section 4.3 we briefly discussed the ODE/IM correspondence for the cigar NLSM. Recall that the correspondence relates the eigenvalues of the chiral transfer-matrices to the connection coefficients for the family of second order differential equations $\mathcal{D}(\theta) \psi=0$ with the operators $\mathcal{D}(\theta)$ of the form (4.51). The generalization of the construction to the sausage model is based on the ideas from the work [27] and goes along the following line.

As far as our attention was confined to the CFT, there was no need to separately consider the antiholomorphic operators, $\overline{\mathcal{D}}(\bar{\theta})=-\partial_{\bar{z}}^{2}+\bar{T}_{\bar{L}}(\bar{z})+\mathrm{e}^{2 \bar{\theta}} \overline{\mathcal{P}}(\bar{z})$, since there was only a nomenclature difference between the holomorphic and antiholomorphic cases. In the massive QFT, following [27], one should substitute the pair $\left(\mathcal{D}\left(\theta_{0}+\theta\right), \overline{\mathcal{D}}\left(\theta_{0}-\theta\right)\right)$ by a pair of $(2 \times 2)$-matrix valued differential operators

$$
\boldsymbol{D}(\theta)=\partial_{z}-\boldsymbol{A}_{z}, \quad \overline{\boldsymbol{D}}(\theta)=\partial_{\bar{z}}-\boldsymbol{A}_{\bar{z}}
$$

with

$$
\begin{aligned}
& \boldsymbol{A}_{z}=-\frac{1}{2} \partial_{z} \eta \sigma_{3}+\sigma_{+} \mathrm{e}^{+\eta}+\sigma_{-} \mathrm{e}^{2 \theta_{0}+2 \theta} \mathcal{P}(z) \mathrm{e}^{-\eta} \\
& \boldsymbol{A}_{\bar{z}}=+\frac{1}{2} \partial_{\bar{z}} \eta \sigma_{3}+\sigma_{-} \mathrm{e}^{+\eta}+\sigma_{+} \mathrm{e}^{2 \theta_{0}-2 \theta} \overline{\mathcal{P}}(\bar{z}) \mathrm{e}^{-\eta},
\end{aligned}
$$

where $\sigma_{3}, \sigma_{ \pm}=\left(\sigma_{1} \pm \mathrm{i} \sigma_{2}\right) / 2$ are the standard Pauli matrices and $\mathcal{P}(z)$ is given by (4.46). In fact, $\left(\boldsymbol{A}_{z}, \boldsymbol{A}_{\bar{z}}\right)$ form a $\mathfrak{s l}(2)$ connection whose flatness is a necessary condition for the existence of a solution to the linear problem

$$
\boldsymbol{D}(\theta) \boldsymbol{\Psi}=0, \quad \overline{\boldsymbol{D}}(\theta) \boldsymbol{\Psi}=0 .
$$

The zero-curvature relation leads to the Modified Sinh-Gordon (MShG) equation:

$$
\partial_{z} \partial_{\bar{z}} \eta-\mathrm{e}^{2 \eta}+\rho^{4}|\mathcal{P}(z)|^{2} \mathrm{e}^{-2 \eta}=0, \quad \rho=\mathrm{e}^{\theta_{0}} .
$$

In refs. [57, 58], a class of singular solutions to this partial differential equation distinguished by special monodromy properties of the associated linear problem (5.71) was introduced. Together with the singularities at $z=z_{1}, z_{2}, z_{3}$, the solutions are allowed to have the socalled apparent singularities, which do not affect the monodromy properties of the auxiliary linear problem (5.71). In the limit $\theta_{0} \rightarrow-\infty$ with $\theta_{+}=\theta_{0}+\theta$ kept fixed, the system (5.71) can be reduced to $\mathcal{D}\left(\theta_{+}\right) \psi=0, \partial_{\bar{z}} \psi=0$ and the apparent singularities manifest themselves as the monodromy free singularities for the operator $\mathcal{D}\left(\theta_{+}\right)$of the form (4.51). Parallel to this, the limit $\theta_{0} \rightarrow-\infty$ with $\theta_{-}=\theta_{0}-\theta$ kept fixed can be considered, which leads to the corresponding antiholomorphic equations $\overline{\mathcal{D}}\left(\theta_{-}\right) \psi=0$ and $\partial_{z} \psi=0$.

In the same works $[57,58]$, evidence was presented that the linear problem (5.71) built from the special singular solutions of the MShG equation makes up the ODE part for the ODE/IQFT correspondence where the IQFT counterpart is the so-called Fateev model [80]. The latter is governed by the Lagrangian

$$
\mathcal{L}=\frac{1}{16 \pi} \sum_{i=1}^{3}\left(\partial_{\mu} \varphi_{i}\right)^{2}+2 \mathcal{M}\left(\mathrm{e}^{\mathrm{i} \alpha_{3} \varphi_{3}} \cos \left(\alpha_{1} \varphi_{1}+\alpha_{2} \varphi_{2}\right)+\mathrm{e}^{-\mathrm{i} \alpha_{3} \varphi_{3}} \cos \left(\alpha_{1} \varphi_{1}-\alpha_{2} \varphi_{2}\right)\right)
$$


for the three scalar fields $\varphi_{i}=\varphi_{i}(t, x)$ which satisfy the periodic boundary conditions

$$
\varphi_{i}(t, x+R)=\varphi_{i}(t, x) .
$$

It is important that the dimensionless coupling constants $\alpha_{i}$ satisfy the linear constraint

$$
\alpha_{1}^{2}+\alpha_{2}^{2}+\alpha_{3}^{2}=\frac{1}{2}
$$

so that the coupling $\mathcal{M}$ in the renormalized Lagrangian (5.73) has the dimensions of mass, $\mathcal{M} \sim$ [mass]. Within the ODE/IQFT correspondence the parameters are identified as follows

$$
a_{i}=4 \alpha_{i}^{2}, \quad(i=1,2,3),
$$

whereas the relation between the dimensionless parameter $\mathcal{M} R$ and $\rho$ from (5.72) is given by

$$
\rho=\frac{1}{2} \mathcal{M} R .
$$

Although the original considerations of refs. [57, 58] were focused on the ODE/IQFT correspondence with all three parameters $a_{1}, a_{2}, a_{3}$ positive, in the subsequent work [81] evidence was presented that the correspondence remains valid with minimum modifications to the case $a_{1}, a_{2}>0$ and $a_{3}<0$. In the recent works [82,83], the same conclusion was reached for $a_{1}, a_{2}>0$ and $a_{3}=0$. Among the tasks of the current paper is to argue that the ODE/IQFT correspondence remains valid for

$$
a_{1}=-n, \quad a_{2}=n+2, \quad a_{3}=0 \quad \text { with } \quad n>0 .
$$

In this case, the coupling $\alpha_{3}$ in the Lagrangian (5.73) vanishes and the field $\varphi_{3}$ is decoupled. The interaction part turns out to be the Lagrangian for the dual action of the sausage model (5.8) provided the identifications $\varphi_{1}=2 \varphi, \varphi_{2}=2 \vartheta$ are made. Notice that with the $m-\mathcal{M}$ relation for the sausage model (5.16), formula (5.77) can be re-written as

$$
\rho=\frac{r}{8 \pi} \text {. }
$$

The ODE/IQFT correspondence suggests that for any common eigenvector $|\psi\rangle \in \mathcal{H}_{k}^{(1)}$ of the commuting family of operators $\mathbb{A}(\theta)$ and $\mathbb{B}(\theta)$, there exists a singular solution of the MShG equation (5.72) with $\mathcal{P}(z)$ given by (4.46) and the parameters $a_{i}$ as in (5.78). The solution should be such that $\mathrm{e}^{-\eta}$ is a smooth, single valued complex function without zeroes on the punctured Riemann sphere. In the vicinity of $z=z_{1}, z_{3}$, the leading behaviour is described by

$$
\mathrm{e}^{-\eta} \sim|\mathcal{P}(z)|^{-\frac{1}{2}} \quad \text { as } \quad\left|z-z_{i}\right| \rightarrow 0,
$$

whereas in the neighbourhood of the second puncture

$$
\mathrm{e}^{-\eta} \sim\left|z-z_{2}\right|^{1-(n+2)|k|} \quad \text { as } \quad\left|z-z_{2}\right| \rightarrow 0
$$

with $0<|k|<\frac{1}{2} \cdot{ }^{15}$ The description of the apparent singularities involves some technical details that are completely analogous to those discussed in ref. [58]. In the case of the

\footnotetext{
${ }^{15}$ At $|k|=0, \frac{1}{2}$ the leading asymptotic (5.81) involves logarithms. Here we ignore such subtleties.
} 
vacuum state, the apparent singularities are absent and the solution $\eta$ is real. Notice that the point $z=\infty$ on the sphere is assumed to be regular, so that

$$
\mathrm{e}^{-\eta} \sim|z|^{2} \quad \text { as } \quad|z| \rightarrow \infty .
$$

As it was mentioned in the previous subsection, the eigenvalue of the operator $\mathbb{S}$ which appears in the large $\theta$-asymptotic formulae (viii) and (ix) is of special interest. Let us introduce the "regularized" value of the solution at the puncture $z=z_{2}$ as

$$
\eta=((n+2)|k|-1) \log \left|z-z_{2}\right|+\eta^{(\mathrm{reg})}+o(1) .
$$

Then for the solution corresponding to the eigenvector $|\psi\rangle \in \mathcal{H}_{k}^{(1)}$ with $0<k<\frac{1}{2}$, the following formula holds:

$$
\mathfrak{S}^{(\psi)}=\left(\frac{\rho}{n+2}\right)^{-2 k} \frac{\Gamma(k)}{\Gamma(1-k)} \frac{\exp \left(\eta^{(\mathrm{reg})}\right)}{(n+2)}\left|\frac{z_{13}}{z_{12} z_{23}}\right|^{-(n+2) k},
$$

where we use the shortcut notation $z_{i j}=z_{i}-z_{j}$.

We can now describe, in precise terms, the ODE/IQFT correspondence for the sausage model. Consider the auxiliary linear problem (5.71) associated with the singular solution of the MShG equation. The puncture $z=z_{2}$ is a regular singular point for this system of ODE. In the vicinity of this point, assuming that $0<k<\frac{1}{2}$, one can introduce the basis solutions by means of the following asymptotic formulae as $z \rightarrow z_{2}$ :

$$
\begin{aligned}
& \Theta_{-}(z, \bar{z} \mid \theta) \rightarrow \frac{\mathrm{e}^{+\mathrm{i} \beta_{2}}}{\sqrt{\sin (2 \pi k)}} \mathrm{e}^{-k\left(\theta-\frac{\mathrm{i} \pi n}{2}\right)}\left(\frac{z-z_{2}}{\bar{z}-\bar{z}_{2}}\right)^{+\frac{1}{4}(1-k(n+2))}\left(\begin{array}{l}
1 \\
0
\end{array}\right) \\
& \Theta_{+}(z, \bar{z} \mid \theta) \rightarrow \frac{\mathrm{e}^{-\mathrm{i} \beta_{2}}}{\sqrt{\sin (2 \pi k)}} \mathrm{e}^{+k\left(\theta-\frac{\mathrm{i} \pi n}{2}\right)}\left(\frac{z-z_{2}}{\bar{z}-\bar{z}_{2}}\right)^{-\frac{1}{4}(1-k(n+2))}\left(\begin{array}{l}
0 \\
1
\end{array}\right)
\end{aligned}
$$

where, for convenience, the constant phase factor is set to be

$$
\mathrm{e}^{\mathrm{i} \beta_{2}}=\left(\frac{z_{12} z_{23}}{z_{13}} \frac{\bar{z}_{13}}{\bar{z}_{12} \bar{z}_{23}}\right)^{\frac{k}{4}(n+2)} .
$$

Unlike $z=z_{2}$, the puncture at $z=z_{1}$ is an irregular singular point for the auxiliary linear problem. In its neighbourhood, and for $\frac{\pi}{2}(n-1) \leq \Im m(\theta) \leq \frac{\pi}{2}(n+1)$, another solution can be uniquely defined using the WKB asymptotic condition:

$$
\begin{aligned}
\boldsymbol{\Xi}(z, \bar{z} \mid \theta) \rightarrow & |\mathcal{P}(z)|^{\frac{1}{4}} \exp \left(-\rho \mathrm{e}^{\theta} \int_{z_{2}}^{z} \mathrm{~d} z \sqrt{\mathcal{P}(z)}-\rho \mathrm{e}^{-\theta} \int_{\bar{z}_{2}}^{\bar{z}} \mathrm{~d} \bar{z} \sqrt{\overline{\mathcal{P}}(\bar{z})}\right) \\
& \times\left(\begin{array}{l}
+\mathrm{e}^{-\frac{\theta}{2}}(\mathcal{P}(z))^{-\frac{1}{4}} \\
-\mathrm{e}^{+\frac{\theta}{2}}(\overline{\mathcal{P}}(\bar{z}))^{-\frac{1}{4}}
\end{array}\right) \quad \text { as } \quad z \rightarrow z_{1} .
\end{aligned}
$$

There must be a linear relation between these three solutions and hence,

$$
\Xi\left(z, \bar{z} \mid \theta+\frac{\mathrm{i} \pi n}{2}\right)=\mathfrak{A}_{+}^{(\psi)}(\theta) \Theta_{-}\left(z, \bar{z} \mid \theta+\frac{\mathrm{i} \pi n}{2}\right)+\mathfrak{A}_{-}^{(\psi)}(\theta) \Theta_{+}\left(z, \bar{z} \mid \theta+\frac{\mathrm{i} \pi n}{2}\right) .
$$


The ODE/IM correspondence states that the connection coefficients $\mathfrak{A}_{+}^{(\psi)}(\theta)$ and $\mathfrak{A}_{-}^{(\psi)}(\theta)$ coincide with the eigenvalues of the operators $\mathbb{A}(\theta)$ and $\mathcal{C} \mathbb{A}(\theta) \mathcal{C}$, for the common eigenvector $|\psi\rangle \in \mathcal{H}_{k}^{(1)}$ associated with the singular solution of the MShG equation. The eigenvalues of the transfer-matrices $\mathbb{T}_{\frac{1}{2}} \equiv \mathbb{T}$, and more generally $\mathbb{T}_{j}$ with $j=\frac{1}{2}, 1, \ldots$, can be obtained by the formulae similar to eqs. (4.39)-(4.41):

$$
\begin{aligned}
\boldsymbol{\Xi}\left(z, \bar{z} \mid \theta+\mathrm{i} \pi\left(2 j+\frac{1}{2}\right)\right)= & \mathfrak{T}_{j}^{(\psi)}(\theta+\mathrm{i} \pi j) \boldsymbol{\Xi}\left(z, \bar{z} \mid \theta+\frac{\mathrm{i} \pi}{2}\right) \\
& -\mathfrak{T}_{j-\frac{1}{2}}^{(\psi)}\left(\theta+\mathrm{i} \pi\left(j+\frac{1}{2}\right)\right) \boldsymbol{\Xi}\left(z, \bar{z} \mid \theta-\frac{\mathrm{i} \pi}{2}\right) .
\end{aligned}
$$

Finally, the eigenvalues of the operators $\mathbb{B}(\theta)$ and $\mathcal{C} \mathbb{B}(\theta) \mathcal{C}$ can also be expressed in terms of certain connection coefficients of the ODE system (5.71). For this purpose, one needs to introduce suitable basis solutions in the vicinity of the third puncture $z=z_{3}$. The corresponding formulae are simple generalizations of (4.59) and we do not present them here.

\section{Discussion}

In this work we considered the problem of the quantization of the sausage NLSM. To conclude, let's summarize and discuss the key results of the paper.

We demonstrated that the flat connection for the classical sausage model admits the ultralocal gauge and thus, "Hamiltonian Methods in the Theory of Solitons" [17] can be applied without modifications. In connection with this, we believe that the problem with ultralocality for other integrable NLSM should be revisited. It would be interesting to see how the integrable canonical structures of ref. [84] fit in with the results of this work.

Since the classical integrable structures in the sausage model turn out to be similar to those from the integrable $\mathrm{KdV}$ /sine-Gordon hierarchy, in our study of the quantum model we closely followed the ideas of the works $[11,18,19]$. We paid special attention to the integrable structures of the cigar NLSM - the CFT governing the ultraviolet behaviour of the quantum sausage. In particular we constructed the BLZ type representation for the chiral transfer-matrices in the quantum cigar.

The chiral transfer-matrices depend on a number of parameters and can be considered in the parameter domain where they are not directly related to the cigar NLSM. In this case, they are still of physical interest since they can be interpreted as the transfer-matrices for the minimal $\mathbb{Z}_{n}$ parafermionic models from ref. [49]. The situation here resembles the interplay between the quantum Liouville theory and the BPZ minimal models. We constructed lattice transfer-matrices and presented numerical evidence that in the scaling limit they become the chiral transfer-matrices in the parafermionic regime. We believe that it may hint as to how to proceed with the lattice formulation of the cigar and sausage models. To go further in this direction the most promising approach is, perhaps, the method of separation of variables [56] which was successfully applied to a similar problem appearing in the quantization of the sinh-Gordon model [26, 85-90]. Another interesting possibility is related to the work [91], where some spectral properties of the cigar NLSM were observed to appear in the scaling limit of a certain inhomogeneous version of the 6 -vertex model. 
One of the most effective methods for the calculation of the spectrum of commuting families of operators including the transfer-matrices in integrable quantum field theory is based on the ODE/IQFT correspondence. From our study of the parafermionic transfermatrix, we proposed the ODE counterpart in the correspondence for the cigar NLSM. It turns out to be identical to that which was introduced earlier in the context of the so-called paperclip model in the works $[42,43]$. Based on the results of these papers, we derived non-linear integral equations for determining the vacuum eigenvalues of the chiral transfermatrix which work both for the cigar and the parafermionic regimes. We believe that this might be a good starting point for applying the powerful fermionic methods [92-96] to the sausage/O(3) NLSM.

In refs. $[27,58]$, a conceptual explanation was given of how the ODE/IQFT correspondence for integrable conformal field theory can be generalized to the massive IQFT. Following this route, we extended the ODE/IQFT correspondence from the cigar to the sausage NLSM. With the correspondence one can uncover the basic integrable structures by studying the properties of the connection coefficients of the ordinary differential equations. The main result of this paper is the list of properties (i)-(x) from section 5.3 for the commuting families of operators in the sausage model which includes the quantum transfer-matrix. The technical result that deserves to be mentioned is the system of NLIE which describes the vacuum eigenvalues of the commuting families of operators. Among other things, it allows one to calculate the $k$-vacuum energies of the sausage/O(3) NLSM.

There are many results in the literature concerning the energy spectrum of the $O(3)$ sigma model in the sector with $k=0$ [76, 97-99]. In ref. [14] a system of TBA equations was proposed which allows one to calculate the ground state energy for $k=0$ and integer values of the dimensionless coupling $n \geq 3$ of the sausage model. Recently Ahn, Balog and Ravanini [70] transformed this system of TBA to a system of three non linear integral equations which, it is affirmed, works for any real positive $n$. Their main assumption is a periodicity condition for the $Q$-function given by eq. (3.16) from that paper. In our investigations, we did not find any trace of a $Q$-function satisfying such a strong periodic condition. Nevertheless, the numerical results presented in figure 2 from that paper seem to be in agreement with the data obtained from the solution of our NLIE (5.30), (5.31) with $k=0$ and $n=1$. This situation needs to be clarified.

Let us briefly touch on some problems which have not been discussed in the text but are directly related to the subject of this work. We did not make any mention of the sausage model with the topological term equal to $\pi$ which is also expected to be an integrable QFT $[14,100]$. Another closely related model is the so-called 3D sausage model introduced by Fateev in [80]. In the work [15], classical integrability was established for a four-parameter family of NLSM with torsion which includes the 3D sausage as a two parameter subfamily. We believe that extending the ODE/IQFT approach to these models will be useful, both as a step in the development of the method, and in terms of applications. Some results in this direction have already been obtained in ref. [81]. There are also the remarkable works [101-103] on toroidal algebras, which are deeply connected to this field.

All the models mentioned above are based on the $\mathfrak{s l}(2)$-algebra and its associated integrable structures. Since the work of Klimcik [104] there has been increasing interest 


\begin{tabular}{|r|c|r|c|}
\hline$n$ & $\tilde{t}_{2}$ & $n$ & $\tilde{t}_{2}$ \\
\hline 1 & 0 & 6 & 0.0658731 \\
2 & $\frac{\sqrt{2}}{48}$ & 7 & 0.0613178 \\
3 & 0.0546105 & 8 & 0.0561029 \\
4 & 0.0661040 & 9 & 0.0509101 \\
5 & 0.0683646 & 10 & 0.0460445 \\
\hline
\end{tabular}

Table 4. Numerical values of $\tilde{t}_{2}$ for $\mathfrak{j}=\mathfrak{m}=0$ (from ref. [43]).

in "deformed" integrable NLSM associated with higher rank Lie algebras [105-108]. The first principle quantization of such theories seems to be a very interesting problem. In the recent works $[109,110]$, an important step in this direction was taken where a one parameter deformation was found of the set of "circular brane" local integrals of motion introduced in ref. [111]. This offers the possibility for the quantization of the deformed $O(N)$ NLSM along the lines of this work.

Perhaps the main motivation for studying NLSM is based on the fact that certain types of SUSY sigma models are at the heart of the celebrated AdS/CFT correspondence, and integrability is an important possibility. In particular, the NLSM associated with the AdS side of the correspondence for $\mathcal{N}=4$ SUSY Yang Mills theory was argued to be integrable $[112,113]$. As briefly discussed in the introduction, the study of the first principles quantization of the NLSM by traditional techniques has proven to be difficult. A similar situation exists with sigma models on supergroups and superspaces, which are expected to provide theoretical descriptions of condensed matter systems with disorder [114]. That is where one is most tempted to try the power of the ODE/IQFT approach.

\section{Acknowledgments}

S. L.'s gratitude goes to A. B. Zamolodchikov for sharing his insights and support.

The research of G.K. and S. L. is supported by the NSF under grant number NSFPHY-1404056.

\section{A Scaling behaviour of discretized chiral transfer-matrices}

To investigate the scaling behaviour of $\mathcal{T}^{(N)}(\mu)$ (3.39)-(3.44), we conducted numerical work for integer $n$ when the discretized operator is a finite dimensional matrix that can be diagonalized by means of the Bethe ansatz (see appendix B for details). We focused only on the vacuum eigenvalue in the sector $\mathcal{H}_{\mathbf{j}-\frac{\mathfrak{m}}{2}}^{(N)}$ and considered the cases with $n=2,3, \ldots, 6$ and all admissible values of $\mathfrak{j}, \mathfrak{m}(3.14)$. Let $\tau^{(\mathrm{vac})}(\lambda)$ be the vacuum eigenvalue of the chiral transfer-matrix in the parafermionic subspace $\mathcal{V}_{j}^{(\mathfrak{m})}$. We expect that it can be obtained from the vacuum eigenvalue of $\mathcal{T}^{(N)}(\mu)$ by using the formula (3.50) which explicitly describes the scaling limit of the discretized operator. To estimate numerical values of $\tau^{(\mathrm{vac})}(\lambda)$ we used data obtained for a set of finite $N$ and then performed a certain interpolation procedure to $N=\infty$. The results were compared with predictions coming from the properties of $\tau^{(\mathrm{vac})}(\lambda)$ 


\begin{tabular}{|c|ccccc|c|}
\hline root \# & $N=501$ & $N=1001$ & $N=1500$ & $N=2600$ & $N=\infty$ & $2 u_{l}^{\frac{n}{2}}$ \\
\hline 1 & 0.4818860 & 0.4818829 & 0.4818820 & 0.4818814 & 0.4818809 & 0.47349 \\
2 & 1.4891566 & 1.4891424 & 1.4891392 & 1.4891372 & 1.4891359 & 1.48725 \\
3 & 2.4919329 & 2.4918863 & 2.4918769 & 2.4918715 & 2.491868 & 2.49093 \\
4 & 3.4935044 & 3.4933890 & 3.4933666 & 3.4933541 & 3.493348 & 3.49276 \\
5 & 4.4946127 & 4.4943769 & 4.4943321 & 4.4943074 & 4.494295 & 4.49387 \\
6 & 5.4955294 & 5.4951073 & 5.4950277 & 5.4949844 & 5.494962 & 5.49464 \\
7 & 6.4963870 & 6.4956974 & 6.4955682 & 6.4954981 & 6.495463 & 6.49521 \\
8 & 7.4972634 & 7.4962107 & 7.4960140 & 7.4959077 & 7.495854 & 7.49564 \\
9 & 8.4982121 & 8.4966857 & 8.4964010 & 8.4962476 & 8.496171 & 8.49599 \\
10 & 9.4992734 & 9.4971480 & 9.4967523 & 9.4965392 & 9.496432 & 9.49628 \\
11 & 10.500481 & 10.497616 & 10.497084 & 10.496797 & 10.49665 & 10.49652 \\
12 & 11.501864 & 11.498105 & 11.497406 & 11.497031 & 11.49684 & 11.49672 \\
13 & 12.503448 & 12.498625 & 12.497729 & 12.497248 & 12.49701 & 12.49690 \\
14 & 13.505258 & 13.499187 & 13.498060 & 13.497455 & 13.49715 & 13.49705 \\
15 & 14.507318 & 14.499799 & 14.498404 & 14.497655 & 14.49728 & 14.49719 \\
\hline
\end{tabular}

Table 5. Numerical values of $\frac{2 N}{\pi}\left[\mu_{l}^{(N)}\right]^{n}$, where $\mu_{l}^{(N)}>0$ are the roots of the vacuum eigenvalue of the discretized operator $\mathcal{T}^{(N)}(\mu)$ for $n=4, \mathfrak{j}=\mathfrak{m}=0$. The column " $N=\infty$ " was obtained by interpolating the results for finite $N$. The entries in the last column were calculated by using the asymptotic formula (A.2) truncated at the first non-zero term in the series.

discussed in section 2.2, specialized to the values $p_{1}=\frac{i}{2} \mathfrak{m}$ and $p_{2}=\mathfrak{j}+\frac{1}{2}$. Agreement was found in all cases considered. In this appendix, some of our numerical work is presented.

Let $\left\{u_{l}\right\}_{l=1}^{\infty}$ be the set of zeroes of $\tau^{(\mathrm{vac})}(\lambda)$ considered as a function of $\lambda^{2}$. From the numerical data it was found that all the zeroes are simple, real, positive, and accumulate towards $\lambda^{2}=\infty$ with the leading asymptotic behaviour

$$
u_{l} \sim\left(\frac{1}{2}\right)^{\frac{2}{n}} \times \begin{cases}\left(l-\frac{1}{2}\right)^{\frac{2}{n}} & \text { for } \quad 0 \leq 2 \mathbf{j}<\frac{n}{2} \\ \left(l-\frac{1}{2}+\frac{n}{n+2}\right)^{\frac{2}{n}} & \text { for } \quad 2 \mathbf{j}=\frac{n}{2} \quad(n-\text { even })\end{cases}
$$

For $0 \leq 2 \mathfrak{j}<\frac{n}{2}$, this is consistent with the asymptotically exact formula,

$$
u_{l}^{\frac{n}{2}}+\frac{1}{2 \pi} \sum_{m=1}^{\infty} \tilde{g}_{m}\left(\frac{\mathrm{i}}{2} \mathfrak{m}, \mathfrak{j}+\frac{1}{2}\right) \sin \left(\frac{2 \pi m}{n+2}\right) u_{l}^{-\frac{n m}{n+2}} \asymp \frac{1}{2}\left(l-\frac{1}{2}\right),
$$

which can be easily derived from eqs. (2.53)-(2.54). Knowledge of the coefficients $\tilde{g}_{m}$ allows us to compute systematic corrections to the leading asymptotic behaviour (A.1). As it follows from eq. (2.52), the first coefficient is

$$
\tilde{g}_{1}\left(p_{1}, p_{2}\right)=\frac{\tilde{t}_{1}\left(p_{1}, p_{2}\right)}{2 \cos \left(\frac{2 \pi p_{2}}{n+2}\right)},
$$

with $\tilde{t}_{1}\left(p_{1}, p_{2}\right)$ - vacuum eigenvalue of $\tilde{\mathfrak{t}}_{1}$ - given by eq. (2.49). Notice that for $p_{2}=$ $\mathfrak{j}+\frac{1}{2}=\frac{n+2}{4}$ (n-even), the denominator in (A.3) is zero so that (A.2) is no longer valid. 

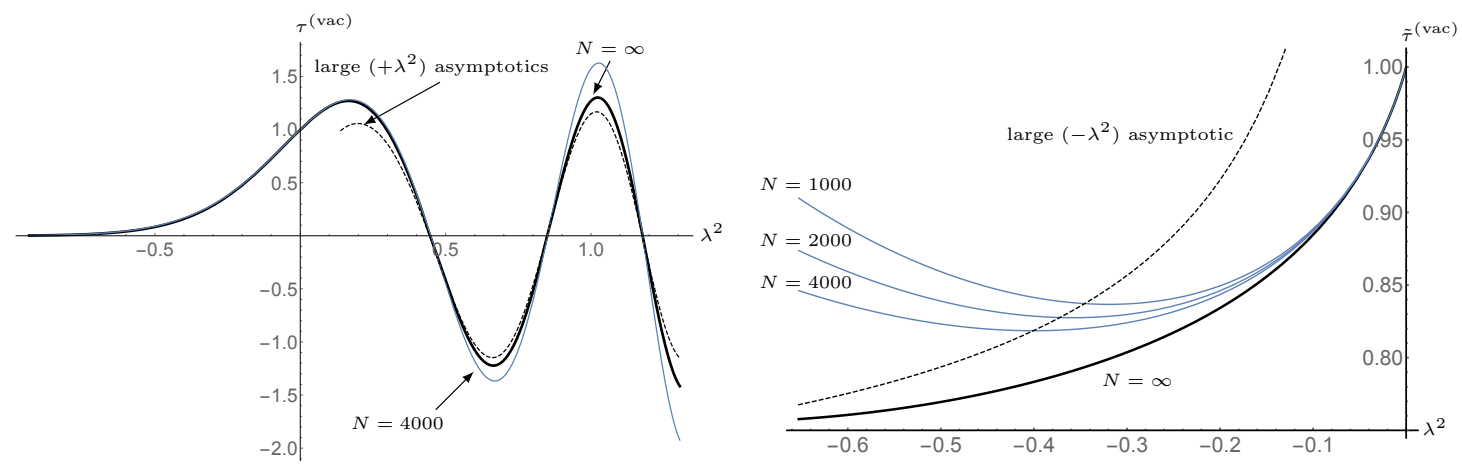

Figure 9. On the left panel, a plot of $\tau^{\text {(vac) }}$ for $n=3,2 \mathfrak{j}=\mathfrak{m}=1$ compared to its large $\left(+\lambda^{2}\right)$ asymptotic following from eq. (2.53). On the right panel, $\tilde{\tau}^{(\mathrm{vac})}=\tau^{\text {(vac) }} \exp \left(2 \pi\left(-\lambda^{2}\right)^{\frac{3}{2}}\right)$ is plotted and compared with the large $\left(-\lambda^{2}\right)$ asymptotic derived from eqs. (2.43), (2.44). The scaling function was numerically estimated by interpolating to $N=\infty$ the data for $N=500,1000,2000,4000$.

Also when $\mathfrak{j}=\mathfrak{m}=0, \tilde{g}_{1}$ vanishes, but for this case the second term in the sum in (A.2) is known, since

$$
\tilde{g}_{2}\left(0, \frac{1}{2}\right)=\frac{\tilde{t}_{2}\left(0, \frac{1}{2}\right)}{2 \cos \left(\frac{\pi}{n+2}\right)}
$$

and numerical values of $\tilde{t}_{2}\left(0, \frac{1}{2}\right)$ were calculated in ref. [43] and are reproduced in table 4 . Truncating the series in (A.2) at the first non vanishing term, we calculated the corrections to the leading asymptotic (A.1). This was compared to the zeroes of the vacuum eigenvalue of $\mathcal{T}^{(N)}(\mu)$ for increasing $N$. In all cases good agreement was observed. As an example, in table 5 the results for $n=4, \mathfrak{j}=\mathfrak{m}=0$ are shown.

As $\lambda^{2} \rightarrow-\infty$, the asymptotic behaviour of $\tau^{\text {(vac) }}$ is dictated by eqs. (2.43), (2.44). Truncating the sum in (2.44) at the first non-zero term and substituting $\tilde{\mathfrak{t}}_{j}$ by its vacuum eigenvalue, we compared this to the results of the $N=\infty$ interpolation. The agreement was good considering that the interpolation procedure becomes rapidly less efficient for increasing values of $\left(-\lambda^{2}\right)$. Figure 9 shows a plot of the estimated scaling function versus the asymptotics for $n=3$ and $2 \mathfrak{j}=\mathfrak{m}=1$.

Another check that can be made is to consider the Taylor expansion of $\tau^{(\mathrm{vac})}(\lambda)$ at zero following from formulae (2.42) and (2.48). The coefficient $t_{1}\left(p_{1}, p_{2}\right)\left(p_{1}=\frac{\mathrm{i}}{2} \mathfrak{m}, p_{2}=\mathfrak{j}+\frac{1}{2}\right)$ can be compared to the corresponding term in the vacuum eigenvalue of the discretized operator:

$$
\mathcal{T}^{(N, \mathrm{vac})}(\mu)=2 \cos \left(\frac{\mathfrak{m} \pi}{n}\right)+t_{1}^{(N)} \mu^{2}+O\left(\mu^{4}\right)
$$

Note that $t_{1}^{(N)}$ is a divergent quantity for large $N$ and must be regularized. According to eq. (3.50), for $n>2$, the following limit exists and converges to $t_{1}$ :

$$
t_{1}\left(\frac{\mathrm{i}}{2} \mathfrak{m}, \mathfrak{j}+\frac{1}{2}\right)=\lim _{N \rightarrow \infty} t_{1}^{(N, \mathrm{reg})}, \quad t_{1}^{(N, \mathrm{reg})}=\left(\frac{\pi}{N}\right)^{\frac{2}{n}}\left(t_{1}^{(N)}+2 N \frac{\cos \left(\frac{\mathfrak{m} \pi}{n}\right)}{\cos \left(\frac{\pi}{n}\right)}\right) .
$$




\begin{tabular}{|c|c|c|c|c|c|c|}
\hline$n=6$ & $N=100$ & $N=200$ & $N=400$ & $N=800$ & $N=\infty$ & eq. (2.48) \\
\hline $2 \mathfrak{j}=\mathfrak{m}=0$ & 0.54474 & 0.54519 & 0.54542 & 0.54553 & 0.5456440 & 0.5456445 \\
\hline $2 \mathfrak{j}=\mathfrak{m}=2$ & 0.43807 & 0.44710 & 0.45357 & 0.45818 & 0.469649 & 0.469446 \\
\hline$n=5$ & $N=100$ & $N=200$ & $N=400$ & $N=750$ & $N=\infty$ & eq. $(2.48)$ \\
\hline $2 \mathfrak{j}=\mathfrak{m}=0$ & 0.86236 & 0.86271 & 0.86287 & 0.86294 & 0.8630048 & 0.8630049 \\
\hline $2 \mathfrak{j}=\mathfrak{m}=2$ & 0.40173 & 0.40751 & 0.41144 & 0.41390 & 0.419808 & 0.419632 \\
\hline
\end{tabular}

Table 6. The regularized value $t_{1}^{(N, \text { reg) }}$ (A.4) for a variety of cases and increasing $N$ compared to the expression for $t_{1}\left(\frac{\mathrm{i}}{2} \mathfrak{m}, \mathfrak{j}+\frac{1}{2}\right)$ given by eq. (2.48). The column " $N=\infty$ " was obtained by interpolation.

We compared the value of $t_{1}\left(\frac{i}{2} \mathfrak{m}, \mathfrak{j}+\frac{1}{2}\right)$ given by eq. (2.48) to the numerical values of $t_{1}^{(N, \text { reg })}$ and found good agreement for $n=3,4 \ldots, 6$ and all the allowed values of $\mathfrak{j}, \mathfrak{m}$. A few cases are presented in table 6.

Finally, let us mention that for $n=2$, analytic expressions exist for both $\tau^{\text {(vac) }}$ and the vacuum eigenvalue of $\mathcal{T}^{(N)}$. In the case $\mathfrak{j}=\mathfrak{m}=0$,

$$
\mathcal{T}^{(N, \mathrm{vac})}(\mu)=2 \prod_{m=1}^{N}\left(1-\mu^{2} \cot \left(\frac{\pi}{2 N}\left(m-\frac{1}{2}\right)\right)\right),
$$

and using the formula (3.50), the scaling limit can be taken explicitly to yield

$$
\tau^{(\text {vac })}(\lambda)=\left(\frac{\mathrm{e}}{2}\right)^{2 \lambda^{2}} \frac{2 \sqrt{\pi}}{\Gamma\left(\frac{1}{2}-2 \lambda^{2}\right)} .
$$

It is easy to verify that this is consistent with the properties of the chiral transfer-matrix discussed in section 2.2. For $n=2$ and $2 \mathfrak{j}=\mathfrak{m}=1$, the discretized operator turns out to be zero for any $N$ and hence, $\tau(\lambda)=0$.

\section{B Scaling behaviour of Bethe roots for the vacuum state}

In this appendix we will consider the vacuum eigenvalue of the matrices $\mathcal{Z}_{ \pm}(\mu)$ in the space $\mathcal{H}_{j-\frac{\mathfrak{m}}{2}}^{(N)}$. Recall that $\mathcal{H}_{\mathbf{j}-\frac{\mathfrak{m}}{2}}^{(N)}$ denotes the eigenspace of the matrix $\pi_{\mathcal{H}^{(N)}}(\mathrm{Z})(3.41),(3.45)$ having eigenvalue $\omega^{\mathfrak{j}-\frac{\mathfrak{m}}{2}}$, where $\mathfrak{j}$ and $\mathfrak{m}$ are restricted as in (3.14). Our considerations are entirely based on the properties of $\mathcal{Z}_{ \pm}(\mu)(\mathrm{i})-(\mathrm{v})$ listed in section 4.1.

Let $\mathcal{Z}_{ \pm}^{(\psi)}(\mu)$ be the eigenvalue corresponding to a common eigenvector $|\psi\rangle$ of the commuting family $\mathcal{Z}_{ \pm}(\mu)$. Using the analytical conditions (iv) and $\mu \rightarrow-\mu$ symmetry (v), it can be written in the form,

$$
\mathcal{Z}_{ \pm}^{(\psi)}(\mu)=B^{(N, \psi)} \mu^{\mathfrak{m}} \prod_{i=1}^{(n-1) N-2 \mathfrak{j}-\mathfrak{m}}\left(1 \mp \frac{\mu}{\mu_{i}}\right) \quad(n-\text { odd })
$$


and

$$
\begin{array}{ll}
\mathcal{Z}_{+}^{(\psi)}(\mu)=B^{(N, \psi)} \mu^{\mathfrak{m}} \prod_{i=1}^{\frac{n N}{2}-\mathfrak{j}-\frac{\mathfrak{m}}{2}}\left(1-\frac{\mu^{2}}{v_{i}}\right) & (n-\text { even }) \\
\mathcal{Z}_{-}^{(\psi)}(\mu)=B^{(N, \psi)} \mu^{\mathfrak{m}} \prod_{i=1}^{\frac{(n-2) N}{2}-\mathfrak{j}-\frac{\mathfrak{m}}{2}}\left(1-\frac{\mu^{2}}{w_{i}}\right) &
\end{array}
$$

From the $T-Q$ type relations (iii), it follows that the overall coefficient $B^{(N, \psi)}$ (depending on the state $|\psi\rangle)$ is the same for both $\mathcal{Z}_{+}^{(\psi)}$ and $\mathcal{Z}_{-}^{(\psi)}$. Another consequence of this relation is that the roots satisfy the following Bethe ansatz equations:

$$
\prod_{i=1}^{(n-1) N-2 \mathfrak{j}-\mathfrak{m}} \frac{\mu_{i}+q^{-1} \mu_{l}}{\mu_{i}+q^{+1} \mu_{l}}=-q^{2 \mathfrak{m}}\left(\frac{1-q^{+\frac{1}{2}} \mu_{l}}{1-q^{-\frac{1}{2}} \mu_{l}}\right)^{2 N} \quad(n-\text { odd })
$$

and

$$
\begin{gathered}
\prod_{i=1}^{\frac{n N}{2}-\mathfrak{j}-\frac{\mathfrak{m}}{2}} \frac{v_{i}-q^{-2} w_{l}}{v_{i}-q^{+2} w_{l}}=-q^{2 \mathfrak{m}} \\
\prod_{i=1}^{\frac{(n-2) N}{2}-\mathfrak{j}-\frac{\mathfrak{m}}{2}} \frac{w_{i}-q^{-2} v_{l}}{w_{i}-q^{+2} v_{l}}=-q^{2 \mathfrak{m}}\left(\frac{1-q^{+1} v_{l}}{1-q^{-1} v_{l}}\right)^{2 N} \quad(n-\text { even })
\end{gathered}
$$

Similar equations for the Fateev-Zamolodchikov spin chain (3.47) with periodic boundary conditions were previously derived in the works [115] and [116] for odd and even $n$, respectively. Notice that the constant $B^{(N, \psi)}$ in (B.1), (B.2) is determined (up to an overall sign) by the quantum Wronskian type relations (ii).

The Bethe ansatz equations are valid for all integer $n \geq 2$ and $\mathfrak{j}, \mathfrak{m}$ restricted to (3.14), except for $2 \mathfrak{j}=\mathfrak{m}=\frac{n}{2}$ ( $n$ even) which requires special attention. In this case, for certain sectors of $\mathcal{H}_{0}^{(N)}$ a significant simplification occurs; $\mathcal{Z}_{-}^{(\psi)}$ vanishes so that the $T-Q$ type relations (iii) become trivial and the quantum Wronskian type relations (ii) can be used to obtain much simpler equations for the roots. For instance, for the vacuum eigenvalue, $\mathcal{Z}_{-}^{(\mathrm{vac})}(\mu)=0$ and $\mathcal{Z}_{+}^{(\mathrm{vac})}$ is given explicitly by

$$
\mathcal{Z}_{+}^{(\mathrm{vac})}(\mu)=2 \sqrt{N} \mu^{\frac{n}{2}} \prod_{l=1}^{N-1}\left(1+\mu^{n} \cot \left(\frac{\pi l}{2 N}\right)\right), \quad\left(2 \mathfrak{j}=\mathfrak{m}=\frac{n}{2}, \quad n-\text { even }\right) .
$$

Recall that the vacuum is defined as the lowest energy state of the Fateev-Zamolodchikov spin chain Hamiltonian (3.47), (3.48), which commutes with both $\mathcal{Z}_{+}(\mu)$ and $\mathcal{Z}_{-}(\mu)$ for any $\mu$.

We studied the solutions to the Bethe ansatz equations corresponding to the low energy states $|\psi\rangle$ of the Fateev-Zamolodchikov spin chain. It was found that the roots accumulate 


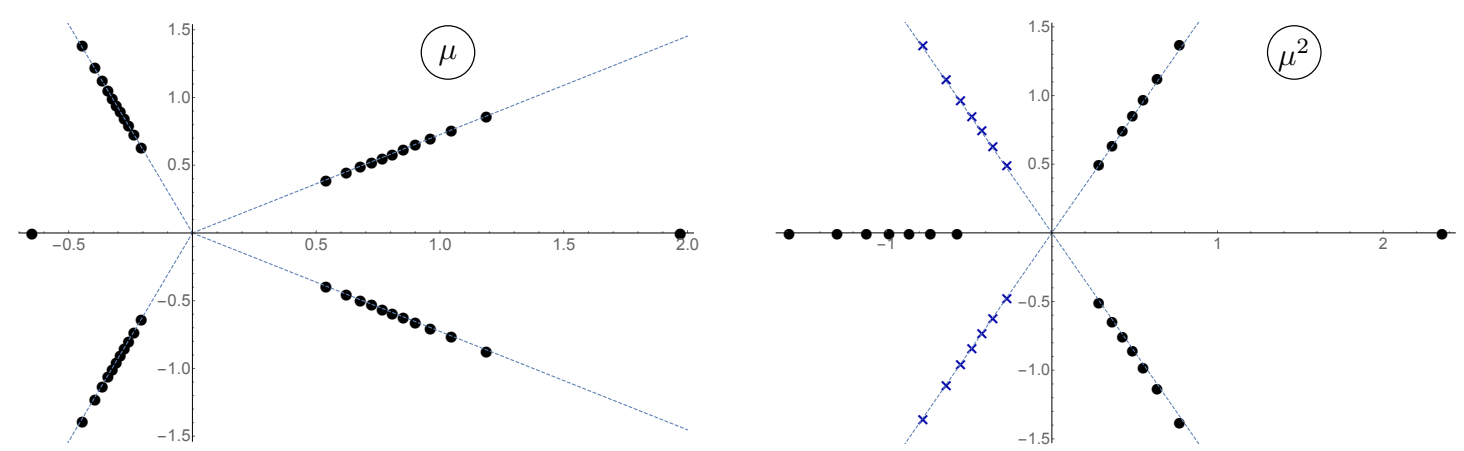

Figure 10. On the left panel, the roots of $\mathcal{Z}_{+}^{(\mathrm{vac})}(\mu)$ are depicted in the complex plane for $n=5$, $\mathfrak{j}=1, \mathfrak{m}=0$ and $N=12$. On the right panel, the roots of $\mathcal{Z}_{+}^{\text {(vac) }}$ (circles) and $\mathcal{Z}_{-}^{\text {(vac) }}$ (crosses) as functions of $\mu^{2}$ are shown for $n=6,2 \mathfrak{j}=3, \mathfrak{m}=1$ and $N=8$.

along the rays given by (see figure 10)

$$
\begin{aligned}
& \arg (\mu)= \pm \frac{\pi}{n} p, \quad p=1,3, \ldots, n-2 \quad\left(\mu_{i}-\text { roots }\right) \\
& \arg \left(\mu^{2}\right)=\frac{2 \pi}{n} p, \quad p=1,3, \ldots, n-1 \quad\left(v_{i}-\operatorname{roots}\right) \\
& \arg \left(\mu^{2}\right)=\frac{2 \pi}{n} p, \quad p=2,4, \ldots, n-2 \quad\left(w_{i}-\text { roots }\right)
\end{aligned}
$$

In the scaling limit most of the roots become densely packed along the rays. However we observed that at the edges of the distribution, the roots exhibit a certain scaling behaviour. In particular, at the edge next to zero of the locus labeled by the integer $p$, with index $i$ enumerating the roots ordered by increasing absolute value, the following limits exist

$$
\lim _{\substack{N \rightarrow \infty \\ i-\text { fixed }}} N^{\frac{1}{n}} \mu_{i, p}^{(N, \psi)}, \quad \lim _{\substack{N \rightarrow \infty \\ i-\text { fixed }}} N^{\frac{2}{n}} v_{i, p}^{(N, \psi)}, \quad \lim _{\substack{N \rightarrow \infty \\ i-\text { fixed }}} N^{\frac{2}{n}} w_{i, p}^{(N, \psi)} .
$$

Here we temporarily exhibit the dependence of the roots on $N$ and the state $|\psi\rangle$. Also, the scaling limit can be defined for the coefficient $B^{(N, \psi)}$ in formulae (B.1), (B.2):

$$
B^{(\psi)}=\operatorname{sim}_{N \rightarrow \infty}(\pi / N)^{\frac{\mathfrak{m}}{n}} B^{(N, \psi)} .
$$

Keeping $N$ finite, consider the logarithm of the r.h.s. of eqs. (4.1) and (4.2) for a given eigenvalue. With $\mathcal{Z}_{ \pm}^{(\psi)}$ of the form (B.1), (B.2) it is straightforward to find their Taylor series at $\lambda=0$. In the case of odd $n$, the expansion coefficients are given by

$$
\begin{array}{ll}
M_{m}^{(N)}=\frac{1}{m}\left(\frac{\pi}{N}\right)^{\frac{m}{n}}\left(\sum_{i} \mu_{i}^{-m}+\frac{(-1)^{m} N}{\cos \left(\frac{\pi m}{2 n}\right)}\right) & (m<n) \\
M_{n}^{(N)}=\frac{\pi}{n N} \sum_{i} \mu_{i}^{-n}+\frac{2}{n}(n-1) \log \left(\frac{N e}{\pi}\right) & \\
M_{m}^{(N)}=\frac{1}{m}\left(\frac{\pi}{N}\right)^{\frac{m}{n}} \sum_{i} \mu_{i}^{-m} & (m>n)
\end{array}
$$


For even $n$,

$$
\begin{array}{rlrl}
V_{m}^{(N)} & =\frac{1}{m}\left(\frac{\pi}{N}\right)^{\frac{2 m}{n}} \sum_{i} v_{i}^{-m} & & (2 m<n) \\
W_{m}^{(N)} & =\frac{1}{m}\left(\frac{\pi}{N}\right)^{\frac{2 m}{n}}\left(\sum_{i} w_{i}^{-m}+\frac{N}{\cos \left(\frac{\pi m}{n}\right)}\right) & (2 m<n) \\
V_{\frac{n}{2}}^{(N)} & =\frac{2 \pi}{n N} \sum_{i} v_{i}^{-\frac{n}{2}}+2 \log \left(\frac{N \mathrm{e}}{\pi}\right) & \\
W_{\frac{n}{2}}^{(N)} & =\frac{2 \pi}{n N} \sum_{i} w_{i}^{-\frac{n}{2}}-\frac{2}{n}(n-2) \log \left(\frac{N \mathrm{e}}{\pi}\right) & \\
V_{m}^{(N)} & =\frac{1}{m}\left(\frac{\pi}{N}\right)^{\frac{2 m}{n}} \sum_{i} v_{i}^{-m}, \quad W_{m}^{(N)}=\frac{1}{m}\left(\frac{\pi}{N}\right)^{\frac{2 m}{n}} \sum_{i} w_{i}^{-m} & & (2 m>n)
\end{array}
$$

It is expected that the following limits exist,

$$
\begin{aligned}
& M_{m}^{(\psi)}=\operatorname{sim}_{N \rightarrow \infty} M_{m}^{(N)} \quad(n-\text { odd }) \\
& V_{m}^{(\psi)}=\operatorname{sim}_{N \rightarrow \infty} V_{m}^{(N)}, \quad W_{m}^{(\psi)}=\operatorname{sim}_{N \rightarrow \infty} W_{m}^{(N)} \quad(n-\text { even })
\end{aligned}
$$

and coincide with the expansion coefficients in $\lambda \equiv \mathrm{e}^{\frac{\theta}{n}}$ of the CFT eigenvalues of $\log \zeta_{ \pm}$:

$$
\begin{array}{ll}
\log \zeta_{ \pm}^{(\psi)}(\theta)=\log B^{(\psi)}+\frac{\mathfrak{m}}{n} \theta \mp \frac{2}{n} \theta \mathrm{e}^{\theta}-\sum_{m=1}^{\infty}( \pm 1)^{m} M_{m}^{(\psi)} \mathrm{e}^{\frac{m \theta}{n}} & (n-\text { odd }) \\
\log \zeta_{+}^{(\psi)}(\theta)=\log B^{(\psi)}+\frac{\mathfrak{m}}{n} \theta-\sum_{l=1}^{\infty} V_{m}^{(\psi)} \mathrm{e}^{\frac{2 m \theta}{n}} & (n-\text { even }) \\
\log \zeta_{-}^{(\psi)}(\theta)=\log B^{(\psi)}+\frac{\mathfrak{m}}{n} \theta+\frac{4}{n} \theta \mathrm{e}^{\theta}-\sum_{m=1}^{\infty} W_{m}^{(\psi)} \mathrm{e}^{\frac{2 m \theta}{n}} . &
\end{array}
$$

Recall that the symbol "slim" stands for the scaling limit which is applied for low energy eigenstates only. For numerical checks, we focused only on the vacuum of the FateevZamolodchikov spin chain (3.47), (3.48). Our numerical work confirmed the existence of the limits (B.9) for $n=3,4, \ldots, 6$ and all admissible values of $\mathfrak{j}$ and $\mathfrak{m}$ (3.14). Since a few of the expansion coefficients in (4.35) are available in explicit form, we have the following analytical predictions for some of the limits in (B.9).

Let $f_{0,1}=f_{0,1}\left(p_{1}, p_{2}\right)$ be defined by eq. (4.33) and $\gamma(x) \equiv \Gamma(x) / \Gamma(1-x)$. Then for $n>2$ one has (here the superscript "(vac)" in the notation for the coefficients (B.9) is 


\begin{tabular}{|c|c|c|c|c|c|c|}
\hline$n=4, \mathfrak{j}=1, \mathfrak{m}=0$ & $N=101$ & $N=201$ & $N=400$ & $N=1001$ & $N=\infty$ & exact \\
\hline$V_{1}^{\text {(vac) }}$ & 2.4301852 & 2.4299253 & 2.4298202 & 2.4297709 & 2.4297498 & 2.4297502 \\
\hline$V_{2}^{\text {(vac) }}-W_{2}^{\text {(vac) }}$ & 3.1094496 & 3.1094648 & 3.1094686 & 3.1094697 & 3.1094699 & 3.1094699 \\
\hline$n=4,2 \mathfrak{j}=\mathfrak{m}=1$ & $N=201$ & $N=401$ & $N=1001$ & $N=1500$ & $N=\infty$ & exact \\
\hline$V_{1}^{\text {(vac) }}$ & -0.970065 & -0.962059 & -0.955825 & -0.954074 & -0.948453 & -0.948425 \\
\hline$W_{1}^{\text {(vac) }}$ & 2.020549 & 2.016718 & 2.013751 & 2.012921 & 2.010289 & 2.010250 \\
\hline$n=5,2 \mathfrak{j}=\mathfrak{m}=2$ & $N=100$ & $N=200$ & $N=400$ & $N=750$ & $N=\infty$ & exact \\
\hline$M_{1}^{\text {(vac) }}$ & -1.09540 & -1.09018 & -1.08669 & -1.08453 & -1.07962 & -1.07956 \\
\hline$M_{2}^{\text {(vac) }}$ & 0.77960 & 0.77649 & 0.77445 & 0.77320 & 0.77054 & 0.77039 \\
\hline
\end{tabular}

Table 7. Numerical values of the coefficients (B.7), (B.8) for the vacuum of the FateevZamolodchikov spin chain (3.47), (3.48). The column $N=\infty$ was obtained by interpolating the finite- $N$ data. The last column lists the exact predictions given in (B.10), (B.11).

omitted):

$$
\begin{aligned}
& M_{m}(\mathfrak{m}, \mathfrak{j})=0 \\
& (m=1,3, \ldots, n-2-2 \mathfrak{m}) \\
& M_{1}\left(\frac{n-1}{2}, \frac{n-1}{4}\right)=-n^{-\frac{1}{n}} \gamma\left(\frac{1}{2}-\frac{1}{2 n}\right) \\
& M_{2}(\mathfrak{m}, \mathfrak{j})=-f_{0,1}\left(-\frac{i \mathfrak{m}}{2}, \mathfrak{j}+\frac{1}{2}\right) \\
& \left(\mathfrak{m}=0,1,2, \ldots, \frac{n-3}{2}\right) \\
& M_{2}\left(\frac{n-1}{2}, \frac{n-1}{4}\right)=-f_{0,1}\left(-\frac{\mathrm{i}(n-1)}{4}, \mathfrak{j}+\frac{1}{2}\right)+\frac{1}{2} n^{-\frac{2}{n}} \gamma^{2}\left(\frac{1}{2}-\frac{1}{2 n}\right) \\
& M_{n}(0, \mathfrak{j})=2 \log \left(\frac{\mathrm{e}}{2}\right)+\frac{2}{n}\left(\gamma_{E}-\log (n)\right)+4 \psi(1+\mathfrak{j})-\psi\left(1+\frac{n}{2}\right)+\gamma_{E} \\
& V_{m}(\mathfrak{m}, \mathfrak{j})=W_{m}(\mathfrak{m}, \mathfrak{j}) \\
& \left(m=1,2, \ldots, \frac{n-2}{2}-\mathfrak{m}\right) \\
& V_{1}(\mathfrak{m}, \mathfrak{j})=W_{1}(\mathfrak{m}, \mathfrak{j})=-f_{0,1}\left(-\frac{\mathrm{i} \mathfrak{m}}{2}, \mathfrak{j}+\frac{1}{2}\right) \quad\left(\mathfrak{m}=0,1,2, \ldots, \frac{n}{2}-2\right) \\
& V_{1}\left(\frac{n-2}{2}, \mathrm{j}\right)=-f_{0,1}\left(-\frac{\mathrm{i}(n-2)}{4}, \mathrm{j}+\frac{1}{2}\right)-n^{-\frac{2}{n}} \gamma\left(\frac{1}{2}-\frac{1}{n}\right) \\
& W_{1}\left(\frac{n-2}{2}, \mathfrak{j}\right)=-f_{0,1}\left(-\frac{\mathrm{i}(n-2)}{4}, \mathfrak{j}+\frac{1}{2}\right)+n^{-\frac{2}{n}} \gamma\left(\frac{1}{2}-\frac{1}{n}\right) \\
& V_{\frac{n}{2}}(0, \mathfrak{j})-W_{\frac{n}{2}}(0, \mathfrak{j})=4 \log \left(\frac{\mathrm{e}}{2}\right)+\frac{4}{n}\left(\gamma_{E}-\log (n)\right)+8 \psi(1+\mathfrak{j})-2 \psi\left(1+\frac{n}{2}\right)+2 \gamma_{E}
\end{aligned}
$$

The numerical data agreed with these explicit formulae. This is shown, for a few cases, in table 7 . 


\begin{tabular}{|c|c|c|c|c|c|c|}
\hline & & & & & & $(-1)^{s} B_{s}(\mathfrak{m})$ \\
\hline \multirow[b]{2}{*}{$n=3,2 \mathfrak{j}=\mathfrak{m}=1$} & $N=101$ & $N=201$ & $N=401$ & $N=1001$ & $N=\infty$ & \multirow[b]{2}{*}{1.626210} \\
\hline & 1.621564 & 1.623528 & 1.624666 & 1.625467 & 1.626213 & \\
\hline \multirow[b]{2}{*}{$n=6,2 \mathfrak{j}=\mathfrak{m}=2$} & $N=100$ & $N=200$ & $N=400$ & $N=800$ & $N=\infty$ & \multirow[b]{2}{*}{1.82536} \\
\hline & 1.79398 & 1.80320 & 1.80971 & 1.81430 & 1.82531 & \\
\hline \multirow[b]{2}{*}{$n=6,2 \mathfrak{j}=3, \mathfrak{m}=1$} & $N=2$ & $N=4$ & $N=6$ & $N=8$ & $N=\infty$ & \multirow[b]{2}{*}{4.10} \\
\hline & 4.178 & 4.148 & 4.135 & 4.127 & 4.08 & \\
\hline
\end{tabular}

Table 8. The absolute value of $(\pi / N)^{\frac{\mathfrak{m}}{n}} B^{(N, v a c)}$ corresponding to the vacuum state of the FateevZamolodchikov spin chain (3.47), (3.48). The column " $N=\infty$ " contains the results of numerical interpolation from the finite $N$ data. The analytical expression for $B_{s}(\mathfrak{m})$ is given by $(4.34)$.

As was already mentioned, the constant $\left(B^{(N, \psi)}\right)^{2}$ can be found using the quantum Wronskian type relations (ii) from section 4.1. The r.h.s. of these relations is proportional to the lattice shift operator $\mathbb{P}^{(N)}$ (3.49) whose eigenvalues are pure phases (3.52). By explicit diagonalization of $\mathcal{Z}_{ \pm}$for small $N$ we found that

$$
\frac{1}{\pi} \arg \left(B^{(N, \psi)}\right)=\frac{1}{n N}((2 \mathfrak{j}-s) s+n(L-\bar{L}))+s \quad(\bmod 2),
$$

where $s=\mathfrak{j}-\frac{1}{2} \mathfrak{m}$ and $L, \bar{L}$ are non-negative integers depending on the state $|\psi\rangle$. For the vacuum state $L=\bar{L}=0$, and the overall sign of the limit $B^{(\text {vac })}$ (B.6) is $(-1)^{s}$. This coincides with the sign factor in $B_{s}(\mathfrak{m})$ (4.34). For large values of $N$, when direct diagonalization becomes impossible, we verified by means of the Bethe ansatz that the absolute value of $(\pi / N)^{\frac{\mathfrak{m}}{n}} B^{(N, \text { vac) }}$ converges to $(-1)^{s} B_{s}(\mathfrak{m})$ (see table 8).

Recall that $2 \mathfrak{j}=\mathfrak{m}=\frac{n}{2}$ with even $n$ is a special case. Using eq. (B.5) the scaling functions can be found explicitly,

$$
\zeta_{+}^{(\mathrm{vac})}(\theta)=\frac{2 \sqrt{\pi} \mathrm{e}^{\frac{\theta}{2}}}{\Gamma\left(1+2 \mathrm{e}^{\theta}\right)}, \quad \zeta_{-}^{(\mathrm{vac})}(\theta)=0 .
$$

This formula can be applied for $n=2$. For the remaining $n=2$ case, $\mathfrak{j}=\mathfrak{m}=0$, it is easy to show that for finite $N$

$$
\mathcal{Z}_{+}^{(\mathrm{vac})}(\mu)=\sqrt{2} \prod_{m=1}^{N}\left(1-\mu^{2} \cot \left(\frac{\pi}{4 N}(2 m-1)\right)\right), \quad \mathcal{Z}_{-}^{(\mathrm{vac})}(\mu)=\sqrt{2},
$$

so that the scaling functions are given by

$$
\zeta_{+}^{\text {vac })}(\theta)=\frac{\sqrt{2 \pi}}{\Gamma\left(\frac{1}{2}+2 \mathrm{e}^{\theta}\right)}\left(\frac{2}{\mathrm{e}}\right)^{2 \mathrm{e}^{\theta}}, \quad \zeta_{-}^{(\mathrm{vac})}(\theta)=\sqrt{2} \exp \left(2 \theta \mathrm{e}^{\theta}\right) .
$$

\section{Derivation of NLIE}

In this appendix we sketch some technical details in the derivation of the system of NLIE (4.94). 
Suppose $\theta, \quad p_{1}^{2}$ and $p_{2}$ are real, then eqs. (4.75), Hermiticity conditions (4.12), (4.62), (4.67) and the periodicity (4.60) imply that

$$
\begin{aligned}
& \mathrm{e}^{-\mathrm{i} \pi \mathrm{e}^{\theta}} {\left[\mathrm{e}^{-\mathrm{i} \pi p_{2}} \beta_{-}(\theta) \alpha_{+}\left(\theta-\frac{\mathrm{i} \pi n}{2}\right)-\mathrm{e}^{+\mathrm{i} \pi p_{2}} \beta_{+}(\theta) \alpha_{-}\left(\theta-\frac{\mathrm{i} \pi n}{2}\right)\right]=} \\
&= \mathrm{e}^{+\mathrm{i} \pi \mathrm{e}^{\theta}}\left[\mathrm{e}^{-\mathrm{i} \pi p_{2}} \beta_{+}(\theta) \alpha_{-}\left(\theta+\frac{\mathrm{i} \pi n}{2}\right)-\mathrm{e}^{+\mathrm{i} \pi p_{2}} \beta_{-}(\theta) \alpha_{+}\left(\theta+\frac{\mathrm{i} \pi n}{2}\right)\right], \quad(\mathrm{C} .1) \\
& \mathrm{e}^{+\mathrm{i} \pi \mathrm{e}^{\theta}}\left[\mathrm{e}^{-\mathrm{i} \pi p_{2}} \beta_{-}(\theta+\mathrm{i} \pi) \alpha_{+}\left(\theta-\frac{\mathrm{i} \pi n}{2}\right)-\mathrm{e}^{+\mathrm{i} \pi p_{2}} \beta_{+}(\theta+\mathrm{i} \pi) \alpha_{-}\left(\theta-\frac{\mathrm{i} \pi n}{2}\right)\right]= \\
&=\mathrm{e}^{-\mathrm{i} \pi \mathrm{e}^{\theta}}\left[\mathrm{e}^{-\mathrm{i} \pi p_{2}} \beta_{+}(\theta+\mathrm{i} \pi) \alpha_{-}\left(\theta+\frac{\mathrm{i} \pi n}{2}\right)-\mathrm{e}^{+\mathrm{i} \pi p_{2}} \beta_{-}(\theta+\mathrm{i} \pi) \alpha_{+}\left(\theta+\frac{\mathrm{i} \pi n}{2}\right)\right] .
\end{aligned}
$$

Due to the analyticity of the operators $\alpha_{ \pm}(\theta)$ and $\beta_{ \pm}(\theta)$, these relations should be satisfied for any complex $\theta$. Let us introduce the shortcut notations

$$
B_{0}=\frac{\beta_{+}(\theta)}{\beta_{-}(\theta)}, \quad B_{1}=\frac{\beta_{+}(\theta+\mathrm{i} \pi)}{\beta_{-}(\theta+\mathrm{i} \pi)}, \quad U=\mathrm{e}^{2 \pi \mathrm{i} p_{2}} \frac{\alpha_{+}\left(\theta+\frac{\mathrm{i} \pi n}{2}\right)}{\alpha_{-}\left(\theta+\frac{\mathrm{i} \pi n}{2}\right)}, \quad A_{ \pm}=\mathrm{e}^{\mp 2 \pi \mathrm{i} p_{2}} \frac{\alpha_{ \pm}\left(\theta-\frac{\mathrm{i} \pi n}{2}\right)}{\alpha_{ \pm}\left(\theta+\frac{\mathrm{i} \pi n}{2}\right)}
$$

and $\Lambda=\exp \left(2 \pi \mathrm{i} \mathrm{e}^{\theta}\right)$. Then (C.1) can be rewritten as

$$
B_{0}=U \frac{1+\Lambda^{-1} A_{+}}{1+\Lambda^{-1} A_{-}}, \quad B_{1}=U \frac{1+\Lambda A_{+}}{1+\Lambda A_{-}} .
$$

Solving these equations w.r.t. $A_{+}$and $A_{-}$, one finds

$$
A_{+}=-\frac{1}{2}\left(\Lambda+\Lambda^{-1}\right)+\frac{\Lambda-\Lambda^{-1}}{B_{1}-B_{0}}\left(B_{0} B_{1} U^{-1}-\frac{1}{2} B_{0}-\frac{1}{2} B_{1}\right)
$$

and similar for $A_{-}$. This formula, combined with the quantum Wronskian relation (4.63) written in the form

$$
\frac{\Lambda-\Lambda^{-1}}{B_{1}-B_{0}}=-\frac{\beta_{-}(\theta) \beta_{-}(\theta+\mathrm{i} \pi)}{2 \mathrm{i} \sin \left(\frac{2 \pi p_{2}}{n+2}\right)}
$$

leads to

$$
A_{+}(\theta)=-\cos \left(2 \pi \mathrm{e}^{\theta}\right)-\frac{\beta_{+}(\theta) \beta_{+}(\theta+\mathrm{i} \pi)}{2 \mathrm{i} \mathrm{U}(\theta) \sin \left(\frac{2 \pi p_{2}}{n+2}\right)}+\frac{\beta_{+}(\theta) \beta_{-}(\theta+\mathrm{i} \pi)+\beta_{-}(\theta) \beta_{+}(\theta+\mathrm{i} \pi)}{4 \mathrm{i} \sin \left(\frac{2 \pi p_{2}}{n+2}\right)} .
$$

Together with the periodicity condition $\beta_{ \pm}(\theta+\mathrm{i} \pi)=\beta_{ \pm}(\theta-\mathrm{i} \pi)$ the last equation implies

$$
A_{+}\left(\theta-\frac{\mathrm{i} \pi}{2}\right)-A_{+}\left(\theta+\frac{\mathrm{i} \pi}{2}\right)=\frac{\beta_{+}\left(\theta+\frac{\mathrm{i} \pi}{2}\right) \beta_{-}\left(\theta-\frac{\mathrm{i} \pi}{2}\right)}{2 \mathrm{i} \sin \left(\frac{2 \pi p_{2}}{n+2}\right)}\left(U^{-1}\left(\theta+\frac{\mathrm{i} \pi}{2}\right)-U^{-1}\left(\theta-\frac{\mathrm{i} \pi}{2}\right)\right) .
$$

As it follows from the quantum Wronskian relation (4.68):

$$
U^{-1}\left(\theta+\frac{\mathrm{i} \pi}{2}\right)-U^{-1}\left(\theta-\frac{\mathrm{i} \pi}{2}\right)=\frac{2 \mathrm{i}^{-2 \pi \mathrm{i} p_{2}} \sin \left(\frac{2 \pi p_{2}}{n+2}\right)}{\alpha_{+}\left(\theta+\frac{\mathrm{i} \pi(n-1)}{2}\right) \alpha_{+}\left(\theta+\frac{\mathrm{i} \pi(n+1)}{2}\right)} .
$$


This can be substituted into the previous formula, yielding eq. (4.76) with the subscript "+". Of course the formula is valid for the "-" case also.

Let us now take a closer look at the second equation in (C.1) specialized to the eigenvalues corresponding to a common eigenvector $|\psi\rangle$. Suppose $\theta_{j}$ is a zero of $\beta_{+}^{(\psi)}(\theta)$. As follows from the quantum Wronskian relation $(4.63), \beta_{-}^{(\psi)}\left(\theta_{j}\right) \neq 0$, and therefore we conclude that

$$
\mathrm{e}^{-\mathrm{i} \pi\left(\mathrm{e}^{\theta_{j}}+p_{2}\right)} \alpha_{+}^{(\psi)}\left(\theta_{j}-\mathrm{i} \pi-\frac{\mathrm{i} \pi n}{2}\right)=-\mathrm{e}^{\mathrm{i} \pi\left(\mathrm{e}^{\theta_{j}}+p_{2}\right)} \alpha_{+}^{(\psi)}\left(\theta_{j}-\mathrm{i} \pi+\frac{\mathrm{i} \pi n}{2}\right),
$$

which can be equivalently written in the form (4.81).

As was mentioned in the main body of the text, the zeroes of the entire periodic function $\beta_{+}^{(\mathrm{vac})}(\theta)=\beta_{+}^{(\mathrm{vac})}(\theta+\mathrm{i} \pi)$ are simple, located on the lines $\Im m(\theta)=\pi(2 m+1), m \in$ $\mathbb{Z}$, and accumulate toward $\Re e(\theta) \rightarrow+\infty$. Also, assuming that the parameters $p_{1}$ and $p_{2}$ are restricted as in cases (b), (c) from section 4.5, it is expected that the entire function $\alpha_{+}^{(\mathrm{vac})}(\theta)$ does not have any zeroes within the strip $|\Im m(\theta)|<\frac{\pi}{2}(n+2)$. Therefore, as follows from the definition (4.77), $\varepsilon^{(\mathrm{vac})}(\theta)$ is an analytic function for $|\Im m(\theta)|<\pi$ where it has the leading asymptotic behaviour (4.79) at $\Re e(\theta) \rightarrow+\infty$. Combining this analytic information with the "quantization condition" (4.82) for the zeroes of $\beta_{+}^{(\mathrm{vac})}(\theta)$ and the asymptotic behaviour (see eq. (4.74))

$$
\log \beta_{+}^{(\mathrm{vac})}(\theta)=-2 \theta \mathrm{e}^{\theta}-k \theta+o(1) \quad \text { as } \quad \Re e(\theta) \rightarrow+\infty \quad|\Im m(\theta)|<\pi,
$$

with $k=\frac{2 p_{2}}{n+2}$, it is a simple exercise (see however appendix D) to derive a dispersion-type relation

$$
\begin{aligned}
& \log \left(\beta_{+}^{(\mathrm{vac})}\left(\theta-\frac{\mathrm{i} \pi}{2}\right) \beta_{+}^{(\mathrm{vac})}\left(\theta+\frac{\mathrm{i} \pi}{2}\right)\right)=2 \pi \mathrm{e}^{\theta}-2 k \theta \\
& -\Im m\left[\int_{-\infty}^{\infty} \frac{\mathrm{d} \theta^{\prime}}{\pi} \frac{1}{1+\mathrm{e}^{2 \theta-2 \theta^{\prime}+2 \mathrm{i} \gamma}}\left(2 L^{(\mathrm{vac})}\left(\theta^{\prime}-\mathrm{i} \gamma\right)+\mathrm{i}\left(\varepsilon^{(\mathrm{vac})}\left(\theta^{\prime}-\mathrm{i} \gamma\right)-4 \pi \mathrm{e}^{\theta^{\prime}-\mathrm{i} \gamma}+2 \pi k\right)\right)\right] .
\end{aligned}
$$

Here $\gamma \in\left(0, \frac{\pi}{2}\right)$ is an arbitrary constant and the notation

$$
L^{(\mathrm{vac})}(\theta)=\log \left(1+\exp \left(-\mathrm{i} \varepsilon^{(\mathrm{vac})}(\theta)\right)\right)
$$

is used.

The next important property employed in the derivation of the system of integral equations (4.94)-(4.96) is that $\varepsilon^{(\mathrm{vac})}(\theta)$ can be written in terms of the Fourier integral

$$
\varepsilon^{(\mathrm{vac})}(\theta)=4 \pi \mathrm{e}^{\theta}-2 \pi k+\int_{\mathbb{R}+\mathrm{i} 0} \frac{\mathrm{d} \nu}{2 \pi} \mathrm{e}^{\mathrm{i} \nu \theta} \tilde{\varepsilon}(\nu) .
$$

Notice that the existence of the Fourier transform is ensured by the asymptotic behaviour (4.79) at $\theta \rightarrow+\infty$, and formulae (4.89), (4.90) for $\theta \rightarrow-\infty$. One can expect that the function $\tilde{\varepsilon}(\nu)$ decays sufficiently fast as $\nu \rightarrow \pm \infty$, so that the integral in (C.4) converges for any $\theta$ in the strip of analyticity $|\Im m(\theta)|<\pi$. It is not difficult to see now 
that

$$
\begin{array}{r}
\log \left[\alpha_{+}^{(\mathrm{vac})}\left(\theta-\frac{\mathrm{i} \pi(n+1)}{2}\right) \alpha_{+}^{(\mathrm{vac})}\left(\theta+\frac{\mathrm{i} \pi(n+1)}{2}\right)\right] \\
=2 \pi \mathrm{e}^{\theta}-2 k \theta+\mathrm{i} \int_{\mathbb{R}+\mathrm{i} 0} \frac{\mathrm{d} \nu}{2 \pi} \mathrm{e}^{\mathrm{i} \nu \theta} \frac{\cosh \left(\frac{\pi(n+1) \nu}{2}\right)}{\sinh \left(\frac{\pi n \nu}{2}\right)} \tilde{\varepsilon}(\nu)
\end{array}
$$

and also that the imaginary part of the function (C.3) with $\theta$ having infinitesimally small negative imaginary part, can be represented by the convergent integral

$$
\Im m\left(L^{(\text {vac })}(\theta-\mathrm{i} 0)\right)=\int_{\mathbb{R}+\mathrm{i} 0} \frac{\mathrm{d} \nu}{2 \pi} \mathrm{e}^{\mathrm{i} \nu \theta} \tilde{L}(\nu) .
$$

Similarly for the function $\omega(\theta)$ (4.87) with $\theta$ real, one has

$$
\omega^{(\text {vac })}(\theta)=4 \pi \mathrm{e}^{\theta}+\int_{\mathbb{R}+\mathrm{i} 0} \frac{\mathrm{d} \nu}{2 \pi} \mathrm{e}^{\mathrm{i} \nu \theta} \tilde{\omega}(\nu), \quad \log \left(1+\mathrm{e}^{-\omega^{(\text {vac })}(\theta)}\right)=\int_{\mathbb{R}+\mathrm{i} 0} \frac{\mathrm{d} \nu}{2 \pi} \mathrm{e}^{\mathrm{i} \nu \theta} \tilde{M}(\nu) .
$$

The remaining part of the derivation of the NLIE consists of straightforward manipulations with the Fourier images $\tilde{\varepsilon}, \tilde{L}, \tilde{\omega}, \tilde{M}$. Finally, going back to functions of the variable $\theta$, one derives the system of integral equations (4.94)-(4.96).

Knowing the functions $\varepsilon^{(\mathrm{vac})}(\theta), \omega^{(\mathrm{vac})}(\theta)$ from the solution of the NLIE, and the asymptotic formulae (4.70), (4.74), one can recover the vacuum eigenvalues of the operators $\alpha_{+}(\theta)$ and $\beta_{+}(\theta)$ from (C.2), (C.5). The corresponding explicit relations are given below, where we drop the superscript "(vac)" like in the NLIE (4.94)-(4.96):

$$
\begin{aligned}
\log \alpha_{+}(\theta)= & -\frac{\pi}{\sin \left(\frac{\pi n}{2}\right)} \mathrm{e}^{\theta}-k \theta+\int_{-\infty}^{\infty} \frac{\mathrm{d} \theta^{\prime}}{2 \pi \mathrm{i}}\left[F_{1}^{(\mathrm{CFT})}\left(\theta-\theta^{\prime}+\mathrm{i} \gamma\right) L\left(\theta^{\prime}-\mathrm{i} \gamma\right)\right. \\
& \left.-F_{1}^{(\mathrm{CFT})}\left(\theta-\theta^{\prime}-\mathrm{i} \gamma\right)\left(L\left(\theta^{\prime}-\mathrm{i} \gamma\right)\right)^{*}\right]+\int_{-\infty}^{\infty} \frac{\mathrm{d} \theta^{\prime}}{\pi} F_{2}\left(\theta-\theta^{\prime}\right) \log \left(1+\mathrm{e}^{-\omega\left(\theta^{\prime}\right)}\right)
\end{aligned}
$$

valid for $|\Im m(\theta)|<\frac{\pi}{2}(n+2)-\gamma$, and

$$
\begin{aligned}
\log \beta_{+}(\theta)= & -2 \theta \mathrm{e}^{\theta}-k \theta+\int_{-\infty}^{\infty} \frac{\mathrm{d} \theta^{\prime}}{2 \pi \mathrm{i}}\left[F_{3}^{(\mathrm{CFT})}\left(\theta-\theta^{\prime}+\mathrm{i} \gamma\right) L\left(\theta^{\prime}-\mathrm{i} \gamma\right)\right. \\
& \left.-F_{3}^{(\mathrm{CFT})}\left(\theta-\theta^{\prime}-\mathrm{i} \gamma\right)\left(L\left(\theta^{\prime}-\mathrm{i} \gamma\right)\right)^{*}\right]-\int_{-\infty}^{\infty} \frac{\mathrm{d} \theta^{\prime}}{\pi} F_{4}\left(\theta-\theta^{\prime}\right) \log \left(1+\mathrm{e}^{-\omega\left(\theta^{\prime}\right)}\right)
\end{aligned}
$$

for $|\Im m(\theta)|<\pi-\gamma$. Here the kernels are given by $F_{1}^{(\mathrm{CFT})}(\theta)=F_{1}(\theta)-\frac{1}{n+2}$, $F_{3}^{(\mathrm{CFT})}(\theta)=F_{3}(\theta)-\frac{1}{n+2}$ with

$$
\begin{aligned}
& F_{1}(\theta)=\frac{1}{n+2} \tanh \left(\frac{\theta}{n+2}\right), \quad F_{2}(\theta)=\frac{\sin \left(\frac{\pi}{n+2}\right)}{2(n+2) \cosh \left(\frac{\theta+\frac{\mathrm{i} \pi}{2}}{n+2}\right) \cosh \left(\frac{\theta-\frac{\mathrm{i} \pi}{2}}{n+2}\right)} \\
& F_{3}(\theta)=\frac{1}{n+2} \operatorname{coth}\left(\frac{\theta}{n+2}\right)-\frac{1}{\sinh (\theta)} \\
& F_{4}(\theta)=\frac{\sin \left(\frac{\pi}{n+2}\right)}{2(n+2) \sinh \left(\frac{\theta+\frac{\mathrm{i} \pi}{2}}{n+2}\right) \sinh \left(\frac{\theta-\frac{\mathrm{i} \pi}{2}}{n+2}\right)}-\frac{1}{2 \cosh (\theta)} .
\end{aligned}
$$


The vacuum eigenvalues of the chiral transfer-matrix can be obtained using the $T-Q$ relation

$$
\tau^{(\mathrm{vac})}(\mathrm{i} \lambda)=\frac{\alpha_{+}(\theta+\mathrm{i} \pi)}{\alpha_{+}(\theta)}+\frac{\alpha_{+}(\theta-\mathrm{i} \pi)}{\alpha_{+}(\theta)} \quad \text { with } \quad \lambda=\mathrm{e}^{\frac{\theta}{n}} .
$$

Combining (C.6), (C.7) with the general asymptotic expansions at $\Re e(\theta) \rightarrow+\infty$ found in $(4.70),(4.74)$, the expressions for the local and dual nonlocal integrals of motion follow

$$
\begin{aligned}
i_{2 m-1}\left(p_{1}, p_{2}\right)= & \int_{-\infty}^{\infty} \frac{\mathrm{d} \theta}{\pi}\left(2 \Im m\left[\mathrm{e}^{(2 m-1)(\theta-\mathrm{i} \gamma)} L(\theta-\mathrm{i} \gamma)\right]+(-1)^{m} \mathrm{e}^{(2 m-1) \theta} \log \left(1+\mathrm{e}^{-\omega(\theta)}\right)\right) \\
\tilde{s}_{m}\left(p_{1}, p_{2}\right)=-\frac{2}{n+2} & \int_{-\infty}^{\infty} \frac{\mathrm{d} \theta}{\pi} \\
& \quad\left(\Im m\left[\mathrm{e}^{\frac{2 m(\theta-\mathrm{i} \gamma)}{n+2}} L(\theta-\mathrm{i} \gamma)\right]-\sin \left(\frac{\pi m}{n+2}\right) \mathrm{e}^{\frac{2 m \theta}{n+2}} \log \left(1+\mathrm{e}^{-\omega(\theta)}\right)\right) .
\end{aligned}
$$

\section{Modified NLIE for oscillating asymptotics}

Here we discuss the modifications to the integral equations (4.94)-(4.96) for the case of real $p_{1} \neq 0$ and $p_{2}>-\frac{1}{2}$, when the asymptotics of the functions $\varepsilon^{(\mathrm{vac})}(\theta)$ and $\omega^{(\mathrm{vac})}(\theta)$ at $\theta \rightarrow-\infty$ oscillate (4.88).

The first important difference in this case is that $\alpha_{+}^{(\mathrm{vac})}(\theta)$ has a set of zeroes $\left\{\theta_{m}^{(\alpha)}\right\}_{m=1}^{\infty}$ in the strip $|\Im m(\theta)|<\frac{\pi}{2}(n+2)$ whose asymptotic behaviour is given by relation (4.93). Secondly, in the derivation of (4.94) presented in the previous appendix, we implicitly assumed that all values $\theta_{*}$ on the real axis, such that $\varepsilon^{(\mathrm{vac})}\left(\theta_{*}\right)=\pi(\bmod 2 \pi)$ arise from the quantization condition (4.82), i.e., $\theta_{*}=\theta_{j}^{(\mathrm{vac})}-\mathrm{i} \pi$ for some $j=1,2, \ldots$ (recall that $\left.\Im m\left(\theta_{j}\right)=\pi\right)$. In other words all such $\theta_{*}$ are related to the zeroes of $\beta_{+}^{(\mathrm{vac})}(\theta)$ and, therefore, form an increasing semi-infinite sequence extending towards $+\infty$ on the real axis (see (4.83)). For the oscillating asymptotics (4.88) this is no longer true. Indeed, it is easy to check from (4.88) that the condition

$$
\varepsilon^{(\text {vac })}\left(\tilde{\theta}_{m}\right)=-\pi(2 m-1) \quad \text { with } \quad m=1,2, \ldots
$$

is satisfied for an infinite set of values $\left\{\tilde{\theta}_{m}\right\}_{m=1}^{\infty}$ which extend towards $-\infty$ such that

$$
\tilde{\theta}_{m}=-\frac{n}{2 p_{1}}\left(\pi m-\frac{1}{2} \delta\left(p_{1}, p_{2}\right)\right)+o\left(\left(m / p_{1}\right)^{-\infty}\right)
$$

valid up to an exponentially small correction. Here

$$
\delta\left(p_{1}, p_{2}\right)=4 p_{1} \theta_{0} / n+\mathrm{i} \log \left[\cos \left(\pi\left(p_{2}+\mathrm{i} p_{1}\right)\right) / \cos \left(\pi\left(p_{2}-\mathrm{i} p_{1}\right)\right]\right.
$$

coincides with the scattering phase defined by eq. (5.11). In the terminology of the Bethe ansatz we have an infinite number of "holes" where the phase passes a resonant value without a corresponding zero $\theta_{j}$. Therefore the integrals in the r.h.s. of (4.94) contain spurious contributions from non-existent roots. To exclude these unwanted contributions one needs to add extra source terms to the r.h.s. of eqs. (4.94). 
Introduce the notation

$$
\begin{aligned}
& J^{(\varepsilon)}(\theta)=-\mathrm{i} \sum_{m=1}^{\infty} \log \left[\frac{S\left(\theta-\tilde{\theta}_{m}\right)}{S\left(\theta+\frac{\mathrm{i} \pi}{2}(n+2)-\theta_{m}^{(\alpha)}\right)}\right] \\
& J^{(\omega)}(\theta)=-\sum_{m=1}^{\infty} \log \left[\frac{t\left(\theta+\frac{\mathrm{i} \pi}{2}-\tilde{\theta}_{m}\right)}{t\left(\theta+\frac{\mathrm{i} \pi}{2}+\frac{\mathrm{i} \pi}{2}(n+2)-\theta_{m}^{(\alpha)}\right)}\right]
\end{aligned}
$$

where $S(\theta)$ and $t(\theta)$ are defined in (E.1) below. Then the modified equations (4.94) can be written as

$$
\begin{aligned}
\varepsilon(\theta-\mathrm{i} \gamma)= & 4 \pi \mathrm{e}^{\theta-\mathrm{i} \gamma}-2 \pi k+J^{(\varepsilon)}(\theta-\mathrm{i} \gamma)+\int_{-\infty}^{\infty} \frac{\mathrm{d} \theta^{\prime}}{2 \pi \mathrm{i}}\left[G\left(\theta-\theta^{\prime}-2 \mathrm{i} \gamma\right)\left(L\left(\theta^{\prime}-\mathrm{i} \gamma\right)\right)^{*}\right. \\
& \left.-G\left(\theta-\theta^{\prime}\right) L\left(\theta^{\prime}-\mathrm{i} \gamma\right)\right]+\int_{-\infty}^{\infty} \frac{\mathrm{d} \theta^{\prime}}{2 \pi} G_{1}\left(\theta-\theta^{\prime}-\mathrm{i} \gamma\right) \log \left(1+\mathrm{e}^{-\omega\left(\theta^{\prime}\right)}\right) \\
\omega(\theta)= & 4 \pi \mathrm{e}^{\theta}+J^{(\omega)}(\theta)+\Im m\left[\int_{-\infty}^{\infty} \frac{\mathrm{d} \theta^{\prime}}{\pi} G_{1}\left(\theta-\theta^{\prime}+\mathrm{i} \gamma\right) L\left(\theta^{\prime}-\mathrm{i} \gamma\right)\right] \\
& -\int_{-\infty}^{\infty} \frac{\mathrm{d} \theta^{\prime}}{\pi} G_{2}\left(\theta-\theta^{\prime}\right) \log \left(1+\mathrm{e}^{-\omega\left(\theta^{\prime}\right)}\right) \\
L(\theta)= & \log \left(1+\mathrm{e}^{-\mathrm{i} \varepsilon(\theta)}\right) .
\end{aligned}
$$

One can check that the leading terms in the asymptotics (4.88) solves these equations at $\theta \rightarrow-\infty$, i.e., when the exponential terms proportional to $\mathrm{e}^{\theta}$ in the r.h.s. are omitted.

\section{E Two-particle $S$-matrix for the sausage model}

Here, we present the explicit form of the two particle scattering amplitudes for the sausage model [14]. The $S$-matrix satisfies the Yang-Baxter equation and was originally introduced as the Boltzmann weights of the so-called 19-vertex model [117].

$$
\begin{aligned}
& S(\theta)=S_{++}^{++}(\theta)=S_{--}^{--}(\theta)=-\frac{\sinh \left(\frac{\mathrm{i} \pi-\theta}{n+2}\right)}{\sinh \left(\frac{\mathrm{i} \pi+\theta}{n+2}\right)} \\
& T(\theta)=S_{+-}^{+-}(\theta)=S_{-+}^{-+}(\theta)=S(\mathrm{i} \pi-\theta) \\
& t(\theta)=S_{+0}^{+0}(\theta)=S_{0+}^{0+}(\theta)=S_{-0}^{-0}(\theta)=S_{0-}^{0-}(\theta)=\frac{\sinh \left(\frac{\theta}{n+2}\right) \sinh \left(\frac{\mathrm{i} \pi-\theta}{n+2}\right)}{\sinh \left(\frac{2 \mathrm{i} \pi-\theta}{n+2}\right) \sinh \left(\frac{\mathrm{i} \pi+\theta}{n+2}\right)} \\
& r(\theta)=a(\mathrm{i} \pi-\theta)=S_{+0}^{0+}(\theta)=S_{0+}^{+0}(\theta)=S_{-0}^{0-}(\theta)=S_{0-}^{-0}(\theta)=S_{00}^{+-}(\mathrm{i} \pi-\theta)=S_{00}^{-+}(\mathrm{i} \pi-\theta) \\
& \quad=S_{+-}^{00}(\mathrm{i} \pi-\theta)=S_{-+}^{00}(\mathrm{i} \pi-\theta)=-\mathrm{i} \frac{\sin \left(\frac{2 \pi}{n+2}\right) \sinh \left(\frac{\mathrm{i} \pi-\theta}{n+2}\right)}{\sinh \left(\frac{2 \mathrm{i} \pi-\theta}{n+2}\right) \sinh \left(\frac{\mathrm{i} \pi+\theta}{n+2}\right)}
\end{aligned}
$$




$$
\begin{aligned}
& R(\theta)=S_{-+}^{+-}(\theta)=S_{+-}^{-+}(\theta)=\frac{\sin \left(\frac{\pi}{n+2}\right) \sin \left(\frac{2 \pi}{n+2}\right)}{\sinh \left(\frac{2 \mathrm{i} \pi-\theta}{n+2}\right) \sinh \left(\frac{\mathrm{i} \pi+\theta}{n+2}\right)} \\
& \sigma(\theta)=S_{00}^{00}(\theta)=S_{+0}^{+0}(\theta)+S_{-+}^{+-}(\theta)
\end{aligned}
$$

As a $9 \times 9$ matrix $\boldsymbol{S}^{(2 \mapsto 2)}$ satisfies the conditions

$$
\begin{aligned}
\left(\boldsymbol{S}^{(2 \mapsto 2)}\right)^{\dagger} \boldsymbol{S}^{(2 \mapsto 2)} & =\boldsymbol{I}^{(2)} \quad \text { for } \Im m(\theta)=0 \\
\operatorname{det} \boldsymbol{S}^{(2 \mapsto 2)}(\theta) & =\left[\frac{\sinh ^{2}\left(\frac{\mathrm{i} \pi-\theta}{n+2}\right)}{\sinh ^{2}\left(\frac{\mathrm{i} \pi+\theta}{n+2}\right)} \frac{\sinh \left(\frac{2 \mathrm{i} \pi+\theta}{n+2}\right)}{\sinh \left(\frac{2 \mathrm{i} \pi-\theta}{n+2}\right)}\right]^{4} .
\end{aligned}
$$

Open Access. This article is distributed under the terms of the Creative Commons Attribution License (CC-BY 4.0), which permits any use, distribution and reproduction in any medium, provided the original author(s) and source are credited.

\section{References}

[1] L.D. Faddeev, E.K. Sklyanin and L.A. Takhtajan, The quantum inverse problem method. 1, Theor. Math. Phys. 40 (1980) 688 [Teor. Mat. Fiz. 40 (1979) 194] [inSPIRE].

[2] R.J. Baxter, Partition function of the eight vertex lattice model, Annals Phys. 70 (1972) 193 [INSPIRE].

[3] R.J. Baxter, Exactly solved models in statistical mechanics, Academic Press, London U.K., (1982) [INSPIRE].

[4] C. Destri and H.J. de Vega, Light cone lattice approach to fermionic theories in $2 D$ : the massive Thirring model, Nucl. Phys. B 290 (1987) 363 [InSPIRE].

[5] K. Pohlmeyer, Integrable Hamiltonian systems and interactions through quadratic constraints, Commun. Math. Phys. 46 (1976) 207 [INSPIRE].

[6] V.E. Zakharov and A.V. Mikhailov, Relativistically invariant two-dimensional models in field theory integrable by the inverse problem technique (in Russian), Sov. Phys. JETP 47 (1978) 1017 [Zh. Eksp. Teor. Fiz. 74 (1978) 1953] [INSPIRE].

[7] A.M. Polyakov and P.B. Wiegmann, Theory of non-Abelian Goldstone bosons, Phys. Lett. B 131 (1983) 121 [INSPIRE].

[8] L.D. Faddeev and N. Yu. Reshetikhin, Integrability of the principal chiral field model in $(1+1)$-dimension, Annals Phys. 167 (1986) 227 [INSPIRE].

[9] A.B. Zamolodchikov and A.B. Zamolodchikov, Factorized $S$ matrices in two-dimensions as the exact solutions of certain relativistic quantum field models, Annals Phys. 120 (1979) 253 [INSPIRE].

[10] A.G. Bytsko, The zero curvature representation for nonlinear $O(3) \sigma$-model, J. Math. Sci. 85 (1994) 1619 [hep-th/9403101] [INSPIRE].

[11] V.V. Bazhanov, S.L. Lukyanov and A.B. Zamolodchikov, Integrable structure of conformal field theory, quantum KdV theory and thermodynamic Bethe ansatz, Commun. Math. Phys. 177 (1996) 381 [hep-th/9412229] [INSPIRE]. 
[12] D. Fioravanti and M. Rossi, A braided Yang-Baxter algebra in a theory of two coupled lattice quantum KdV: algebraic properties and ABA representations, J. Phys. A 35 (2002) 3647 [hep-th/0104002] [INSPIRE].

[13] D. Fioravanti and M. Rossi, Exact conserved quantities on the cylinder 1: conformal case, JHEP 07 (2003) 031 [hep-th/0211094] [INSPIRE].

[14] V.A. Fateev, E. Onofri and A.B. Zamolodchikov, The sausage model (integrable deformations of $O(3) \sigma$-model), Nucl. Phys. B 406 (1993) 521 [inSPIRE].

[15] S.L. Lukyanov, The integrable harmonic map problem versus Ricci flow, Nucl. Phys. B $\mathbf{8 6 5}$ (2012) 308 [arXiv: 1205.3201] [INSPIRE].

[16] E.K. Sklyanin, On the complete integrability of the Landau-Lifshitz equation, LOMI-E-79-3, Russia, (1980).

[17] L.D. Faddeev and L.A. Takhtajan, Hamiltonian methods in the theory of solitons, Springer-Verlag, Berlin Germany, (1987) [INSPIRE].

[18] V.V. Bazhanov, S.L. Lukyanov and A.B. Zamolodchikov, Integrable structure of conformal field theory II. Q-operator and DDV equation, Commun. Math. Phys. 190 (1997) 247 [hep-th/9604044] [INSPIRE].

[19] V.V. Bazhanov, S.L. Lukyanov and A.B. Zamolodchikov, Integrable structure of conformal field theory III. The Yang-Baxter relation, Commun. Math. Phys. 200 (1999) 297 [hep-th/9805008] [INSPIRE].

[20] A. Voros, Spectral zeta functions, Adv. Stud. Pure Math. 21 (1992) 327.

[21] A. Voros, An exact solution method for $1 D$ polynomial Schrödinger equations, J. Phys. A 32 (1999) 5993 [math-ph/9902016].

[22] P. Dorey and R. Tateo, Anharmonic oscillators, the thermodynamic Bethe ansatz and nonlinear integral equations, J. Phys. A 32 (1999) L419 [hep-th/9812211] [InSPIRE].

[23] V.V. Bazhanov, S.L. Lukyanov and A.B. Zamolodchikov, Spectral determinants for Schrödinger equation and Q-operators of conformal field theory, J. Statist. Phys. 102 (2001) 567 [hep-th/9812247] [INSPIRE].

[24] J. Suzuki, Functional relations in Stokes multipliers and solvable models related to $U_{q}\left(A_{n}^{(1)}\right)$, J. Phys. A 33 (2000) 3507 [hep-th/9910215] [inSPIRE].

[25] V.V. Bazhanov, S.L. Lukyanov and A.B. Zamolodchikov, Higher level eigenvalues of Q-operators and Schrödinger equation, Adv. Theor. Math. Phys. 7 (2003) 711 [hep-th/0307108] [INSPIRE].

[26] Al.B. Zamolodchikov, Generalized Mathieu equation and Liouville TBA, in Quantum field theories in two dimensions, collected works of Alexei Zamolodchikov, volume 2, A. Belavin, Ya. Pugai and A. Zamolodchikov eds., World Scientific, Singapore, (2012).

[27] S.L. Lukyanov and A.B. Zamolodchikov, Quantum sine(h)-Gordon model and classical integrable equations, JHEP 07 (2010) 008 [arXiv: 1003.5333] [INSPIRE].

[28] P. Dorey, C. Dunning and R. Tateo, The ODE/IM correspondence, J. Phys. A 40 (2007) R205 [hep-th/0703066] [INSPIRE].

[29] P. Dorey, S. Faldella, S. Negro and R. Tateo, The Bethe ansatz and the Tzitzeica-Bullough-Dodd equation, Phil. Trans. Roy. Soc. Lond. A 371 (2013) 20120052 [arXiv: 1209.5517] [INSPIRE]. 
[30] P. Adamopoulou and C. Dunning, Bethe ansatz equations for the classical $A_{n}^{(1)}$ affine Toda field theories, J. Phys. A 47 (2014) 205205 [arXiv:1401.1187] [INSPIRE].

[31] K. Ito and C. Locke, ODE/IM correspondence and Bethe ansatz for affine Toda field equations, Nucl. Phys. B 896 (2015) 763 [arXiv:1502.00906] [INSPIRE].

[32] D. Masoero, A. Raimondo and D. Valeri, Bethe ansatz and the spectral theory of affine Lie algebra-valued connections I. The simply-laced case, Commun. Math. Phys. 344 (2016) 719 [arXiv: 1501.07421] [INSPIRE].

[33] D. Masoero, A. Raimondo and D. Valeri, Bethe ansatz and the spectral theory of affine Lie algebra-valued connections II. The non simply-laced case, Commun. Math. Phys. 349 (2017) 1063 [arXiv: 1511.00895] [INSPIRE].

[34] K. Ito and H. Shu, ODE/IM correspondence for modified $B_{2}^{(1)}$ affine Toda field equation, Nucl. Phys. B 916 (2017) 414 [arXiv:1605.04668] [InSPIRE].

[35] C. Babenko and F. Smirnov, Suzuki equations and integrals of motion for supersymmetric CFT, Nucl. Phys. B 924 (2017) 406 [arXiv:1706.03349] [INSPIRE].

[36] R.S. Hamilton, The Ricci flow on surfaces, Contemp. Math. 71, Amer. Math. Soc., Providence RI U.S.A., (1988), pg. 237.

[37] S. Elitzur, A. Forge and E. Rabinovici, Some global aspects of string compactifications, Nucl. Phys. B 359 (1991) 581 [InSPIRE].

[38] E. Witten, On string theory and black holes, Phys. Rev. D 44 (1991) 314 [INSPIRE].

[39] Yu. G. Stroganov, A new calculation method for partition functions in some lattice models, Phys. Lett. A 74 (1979) 116 [INSPIRE].

[40] R.J. Baxter and P.A. Pearce, Hard hexagons: interfacial tension and correlation length, J. Phys. A 15 (1982) 897.

[41] A.N. Kirillov and N.Y. Reshetikhin, Exact solution of the integrable XXZ Heisenberg model with arbitrary spin I. The ground state and the excitation spectrum, J. Phys. A 20 (1987) 1565 [INSPIRE].

[42] S.L. Lukyanov, E.S. Vitchev and A.B. Zamolodchikov, Integrable model of boundary interaction: the paperclip, Nucl. Phys. B 683 (2004) 423 [hep-th/0312168] [INSPIRE].

[43] S.L. Lukyanov, A.M. Tsvelik and A.B. Zamolodchikov, Paperclip at $\theta=\pi$, Nucl. Phys. B 719 (2005) 103 [hep-th/0501155] [INSPIRE].

[44] S.L. Lukyanov and A.B. Zamolodchikov, Integrability in 2D field theory/sigma models, lecture notes for the Les Houches school, to appear, France, (2016).

[45] I. Bakas and E. Kiritsis, Beyond the large- $N$ limit: nonlinear $W_{\infty}$ as symmetry of the $\mathrm{SL}(2, R) / \mathrm{U}(1)$ coset model, Int. J. Mod. Phys. A 7S1A (1992) 55 [hep-th/9109029] [INSPIRE].

[46] R. Dijkgraaf, H.L. Verlinde and E.P. Verlinde, String propagation in a black hole geometry, Nucl. Phys. B 371 (1992) 269 [INSPIRE].

[47] A.B. Zamolodchikov and Al.B. Zamolodchikov, unpublished notes, (1995).

[48] A. Gerasimov, A. Marshakov and A. Morozov, Free field representation of parafermions and related coset models, Nucl. Phys. B 328 (1989) 664 [INSPIRE]. 
[49] V.A. Fateev and A.B. Zamolodchikov, Parafermionic currents in the two-dimensional conformal quantum field theory and selfdual critical points in $Z_{N}$ invariant statistical systems, Sov. Phys. JETP 62 (1985) 215 [Zh. Eksp. Teor. Fiz. 89 (1985) 380] [inSPIRE].

[50] V.A. Fateev and A.B. Zamolodchikov, Selfdual solutions of the star triangle relations in $Z_{N}$ models, Phys. Lett. A 92 (1982) 37 [INSPIRE].

[51] G. Felder, BRST approach to minimal models, Nucl. Phys. B 317 (1989) 215 [Erratum ibid. B 324 (1989) 548] [INSPIRE].

[52] A.G. Izergin and V.E. Korepin, The lattice quantum sine-Gordon model, Lett. Math. Phys. 5 (1981) 199 [INSPIRE].

[53] E.K. Sklyanin, Some algebraic structures connected with the Yang-Baxter equation. Representations of quantum algebras, Funct. Anal. Appl. 17 (1983) 273 [Funkt. Anal. Pril. 17 (1983) 34] [INSPIRE].

[54] V.V. Bazhanov and Yu. G. Stroganov, Chiral Potts model as a descendant of the six vertex model, J. Statist. Phys. 59 (1990) 799 [inSPIRE].

[55] R.J. Baxter, V.V. Bazhanov and J.H.H. Perk, Functional relations for transfer matrices of the chiral Potts model, Int. J. Mod. Phys. B 4 (1990) 803 [inSPIRE].

[56] E.K. Sklyanin, Separation of variables - new trends, Prog. Theor. Phys. Suppl. 118 (1995) 35 [solv-int/9504001] [INSPIRE].

[57] S.L. Lukyanov, ODE/IM correspondence for the Fateev model, JHEP 12 (2013) 012 [arXiv:1303.2566] [INSPIRE].

[58] V.V. Bazhanov and S.L. Lukyanov, Integrable structure of quantum field theory: classical flat connections versus quantum stationary states, JHEP 09 (2014) 147 [arXiv:1310.4390] [INSPIRE].

[59] D. Fioravanti, Geometrical loci and CFTs via the Virasoro symmetry of the $m K d V$-SG hierarchy: an excursus, Phys. Lett. B 609 (2005) 173 [hep-th/0408079] [INSPIRE].

[60] B. Feigin and E. Frenkel, Quantization of soliton systems and Langlands duality, Adv. Stud. Pure Math. 61 (2011) 185 [arXiv:0705.2486] [INSPIRE].

[61] A. Klumper, M.T. Batchelor and P.A. Pearce, Central charges of the 6- and 19-vertex models with twisted boundary conditions, J. Phys. A 24 (1991) 3111 [InSPIRE].

[62] C. Destri and H.J. de Vega, New approach to thermal Bethe ansatz, Phys. Rev. Lett. 69 (1992) 2313 [hep-th/9203064] [INSPIRE].

[63] C. Destri and H.J. De Vega, Unified approach to thermodynamic Bethe ansatz and finite size corrections for lattice models and field theories, Nucl. Phys. B 438 (1995) 413 [hep-th/9407117] [INSPIRE].

[64] A.B. Zamolodchikov and A.B. Zamolodchikov, Structure constants and conformal bootstrap in Liouville field theory, Nucl. Phys. B 477 (1996) 577 [hep-th/9506136] [INSPIRE].

[65] V.A. Fateev, I.V. Frolov and A.S. Shvarts, Quantum fluctuations of instantons in the nonlinear $\sigma$-model, Nucl. Phys. B 154 (1979) 1 [INSPIRE].

[66] A.P. Bukhvostov and L.N. Lipatov, Instanton-anti-instanton interaction in the $O(3)$ nonlinear $\sigma$ model and an exactly soluble fermion theory, Nucl. Phys. B 180 (1981) 116 [Pisma Zh. Eksp. Teor. Fiz. 31 (1980) 138] [inSPIRE]. 
[67] M. Lüscher, Does the topological susceptibility in lattice $\sigma$-models scale according to the perturbative renormalization group?, Nucl. Phys. B 200 (1982) 61 [INSPIRE].

[68] M. Lüscher, Volume dependence of the energy spectrum in massive quantum field theories I. Stable particle states, Commun. Math. Phys. 104 (1986) 177 [inSPIRE].

[69] M. Lüscher, Volume dependence of the energy spectrum in massive quantum field theories II. Scattering states, Commun. Math. Phys. 105 (1986) 153 [InSPIRE].

[70] C. Ahn, J. Balog and F. Ravanini, NLIE for the sausage model, arXiv:1701.08933 [INSPIRE].

[71] A.M. Polyakov, Interaction of Goldstone particles in two-dimensions. Applications to ferromagnets and massive Yang-Mills fields, Phys. Lett. B 59 (1975) 79 [INSPIRE].

[72] S. Hikami and E. Brézin, Three loop calculations in the two-dimensional nonlinear $\sigma$-model, J. Phys. A 11 (1978) 1141 [inSPIRE].

[73] P. Hasenfratz, M. Maggiore and F. Niedermayer, The exact mass gap of the $O(3)$ and $O(4)$ nonlinear $\sigma$-models in $D=2$, Phys. Lett. B 245 (1990) 522 [InSPIRE].

[74] M. Lüscher, P. Weisz and U. Wolff, A numerical method to compute the running coupling in asymptotically free theories, Nucl. Phys. B 359 (1991) 221 [INSPIRE].

[75] D.-S. Shin, A determination of the mass gap in the $O(N) \sigma$-model, Nucl. Phys. B 496 (1997) 408 [hep-lat/9611006] [INSPIRE].

[76] J. Balog and A. Hegedus, The finite size spectrum of the 2-dimensional $O(3)$ nonlinear $\sigma$-model, Nucl. Phys. B 829 (2010) 425 [arXiv:0907.1759] [INSPIRE].

[77] G.V. Dunne and M. Ünsal, Resurgence and trans-series in quantum field theory: the $C P^{N-1}$ model, JHEP 11 (2012) 170 [arXiv:1210.2423] [INSPIRE].

[78] A. Dabholkar, Strings on a cone and black hole entropy, Nucl. Phys. B 439 (1995) 650 [hep-th/9408098] [INSPIRE].

[79] V.A. Fateev and A.B. Zamolodchikov, Integrable perturbations of $Z_{N}$ parafermion models and $O(3) \sigma$-model, Phys. Lett. B 271 (1991) 91 [inSPIRE].

[80] V.A. Fateev, The $\sigma$-model (dual) representation for a two-parameter family of integrable quantum field theories, Nucl. Phys. B 473 (1996) 509 [INSPIRE].

[81] V.V. Bazhanov, G.A. Kotousov and S.L. Lukyanov, Winding vacuum energies in a deformed O(4) $\sigma$-model, Nucl. Phys. B 889 (2014) 817 [arXiv:1409.0449] [InSPIRE].

[82] V.V. Bazhanov, S.L. Lukyanov and B.A. Runov, Vacuum energy of the Bukhvostov-Lipatov model, Nucl. Phys. B 911 (2016) 863 [arXiv:1607.04839] [InSPIRE].

[83] V.V. Bazhanov, S.L. Lukyanov and B.A. Runov, Bukhvostov-Lipatov model and quantum-classical duality, arXiv:1711.09021 [INSPIRE].

[84] J.M. Maillet, New integrable canonical structures in two-dimensional models, Nucl. Phys. B 269 (1986) 54 [INSPIRE].

[85] F.A. Smirnov, Quasiclassical study of form-factors in finite volume, hep-th/9802132 [INSPIRE].

[86] S.L. Lukyanov, Finite temperature expectation values of local fields in the sinh-Gordon model, Nucl. Phys. B 612 (2001) 391 [hep-th/0005027] [INSPIRE]. 
[87] A.B. Zamolodchikov, On the thermodynamic Bethe ansatz equation in sinh-Gordon model, J. Phys. A 39 (2006) 12863 [hep-th/0005181] [INSPIRE].

[88] A.G. Bytsko and J. Teschner, Quantization of models with non-compact quantum group symmetry: modular XXZ magnet and lattice sinh-Gordon model, J. Phys. A 39 (2006) 12927 [hep-th/0602093] [INSPIRE].

[89] J. Teschner, On the spectrum of the sinh-Gordon model in finite volume, Nucl. Phys. B 799 (2008) 403 [hep-th/0702214] [INSPIRE].

[90] G. Borot, A. Guionnet and K.K. Kozlowski, Asymptotic expansion of a partition function related to the sinh-model, arXiv:1412.7721 [INSPIRE].

[91] Y. Ikhlef, J.L. Jacobsen and H. Saleur, An integrable spin chain for the $\mathrm{SL}(2, R) / \mathrm{U}(1)$ black hole $\sigma$-model, Phys. Rev. Lett. 108 (2012) 081601 [arXiv:1109.1119] [INSPIRE].

[92] H. Boos, M. Jimbo, T. Miwa, F. Smirnov and Y. Takeyama, Hidden Grassmann structure in the XXZ model, Commun. Math. Phys. 272 (2007) 263 [hep-th/0606280] [INSPIRE].

[93] H. Boos, M. Jimbo, T. Miwa, F. Smirnov and Y. Takeyama, Hidden Grassmann structure in the XXZ model II: creation operators, Commun. Math. Phys. 286 (2009) 875 [arXiv:0801.1176] [INSPIRE].

[94] M. Jimbo, T. Miwa and F. Smirnov, Hidden Grassmann structure in the XXZ model III: introducing Matsubara direction, J. Phys. A 42 (2009) 304018 [arXiv:0811.0439] [INSPIRE].

[95] H. Boos, M. Jimbo, T. Miwa and F. Smirnov, Hidden Grassmann structure in the XXZ model IV: CFT limit, Commun. Math. Phys. 299 (2010) 825 [arXiv:0911.3731] [inSPIRE].

[96] M. Jimbo, T. Miwa and F. Smirnov, Hidden Grassmann structure in the XXZ model V: sine-Gordon model, Lett. Math. Phys. 96 (2011) 325 [arXiv: 1007.0556] [InSPIRE].

[97] P.B. Wiegmann, On the theory of non-Abelian Goldstone bosons in two-dimensions: exact solution of the $O(3)$ nonlinear $\sigma$ model, Phys. Lett. B 141 (1984) 217 [INSPIRE].

[98] J. Balog and A. Hegedus, TBA equations for excited states in the $O(3)$ and $O(4)$ nonlinear $\sigma$-model, J. Phys. A 37 (2004) 1881 [hep-th/0309009] [INSPIRE].

[99] N. Gromov, V. Kazakov and P. Vieira, Finite volume spectrum of $2 D$ field theories from Hirota dynamics, JHEP 12 (2009) 060 [arXiv:0812.5091] [INSPIRE].

[100] A.B. Zamolodchikov and A.B. Zamolodchikov, Massless factorized scattering and $\sigma$-models with topological terms, Nucl. Phys. B 379 (1992) 602 [InSPIRE].

[101] B. Feigin, M. Jimbo, T. Miwa and E. Mukhin, Quantum toroidal $\mathfrak{g l}_{1}$ and Bethe ansatz, J. Phys. A 48 (2015) 244001 [arXiv: 1502.07194] [InSPIRE].

[102] M.N. Alfimov and A.V. Litvinov, On spectrum of ILW hierarchy in conformal field theory II: coset CFT's, JHEP 02 (2015) 150 [arXiv:1411.3313] [INSPIRE].

[103] B. Feigin, M. Jimbo and E. Mukhin, Integrals of motion from quantum toroidal algebras, J. Phys. A 50 (2017) 464001 [arXiv:1705.07984].

[104] C. Klimčík, Integrability of the bi-Yang-Baxter $\sigma$-model, Lett. Math. Phys. 104 (2014) 1095 [arXiv: 1402.2105] [INSPIRE].

[105] B. Hoare, R. Roiban and A.A. Tseytlin, On deformations of $A d S_{n} \times S^{n}$ supercosets, JHEP 06 (2014) 002 [arXiv: 1403.5517] [INSPIRE]. 
[106] F. Delduc, M. Magro and B. Vicedo, Integrable double deformation of the principal chiral model, Nucl. Phys. B 891 (2015) 312 [arXiv:1410.8066] [INSPIRE].

[107] K. Sfetsos, K. Siampos and D.C. Thompson, Generalised integrable $\lambda$ - and $\eta$-deformations and their relation, Nucl. Phys. B 899 (2015) 489 [arXiv: 1506.05784] [InSPIRE].

[108] C. Klimčík, Poisson-Lie T-duals of the bi-Yang-Baxter models, Phys. Lett. B 760 (2016) 345 [arXiv: 1606.03016] [INSPIRE].

[109] A. Litvinov and L. Spodyneiko, On W algebras commuting with a set of screenings, JHEP 11 (2016) 138 [arXiv: 1609.06271] [INSPIRE].

[110] V. Fateev, A. Litvinov and L. Spodyneiko, private communications, to be published.

[111] S.L. Lukyanov and A.B. Zamolodchikov, Integrable circular brane model and Coulomb charging at large conduction, J. Stat. Mech. 0405 (2004) P05003 [hep-th/0306188] [INSPIRE].

[112] I. Bena, J. Polchinski and R. Roiban, Hidden symmetries of the $A d S_{5} \times S^{5}$ superstring, Phys. Rev. D 69 (2004) 046002 [hep-th/0305116] [inSPIRE].

[113] N. Beisert et al., Review of AdS/CFT integrability: an overview, Lett. Math. Phys. 99 (2012) 3 [arXiv: 1012.3982] [INSPIRE].

[114] K.B. Efetov, Supersymmetry in disorder and chaos, Cambridge University Press, New York U.S.A., (1997).

[115] G. Albertini, Bethe-ansatz type equations for the Fateev-Zamolodchikov spin model, J. Phys. A 25 (1992) 1799.

[116] S. Ray, Bethe ansatz study for ground state of Fateev-Zamolodchikov model, J. Math. Phys. 38 (1997) 1524.

[117] A.B. Zamolodchikov and V.A. Fateev, Model factorized $S$ matrix and an integrable Heisenberg chain with spin 1 (in Russian), Sov. J. Nucl. Phys. 32 (1980) 298 [Yad. Fiz. 32 (1980) 581] [INSPIRE]. 